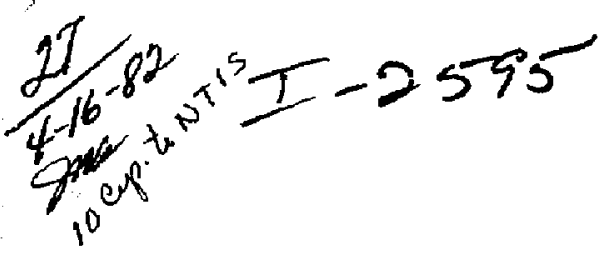

\section{1h. 429}

UCID-1934?

\section{NOTICE}

POAMOHS OF THIS REPORT ARE ILLEGIBLE:

It has been reproduced from the best avaliable copy to permit the broadesi possible avallability.

\title{
NEGATIVE-POTENTIAL OPERATION OF TMX-UPGRADE
}

P. Poulsen, 5. L. Allen, D. E. Baldwin,

T. A. Casper, F. H. Coensgen, D. P. Grubb,

R. 5. Hornady, W. M. Hevins, W. E. Nexsen,

G. D. Porter, T. C. Simonen, G. R. Smith

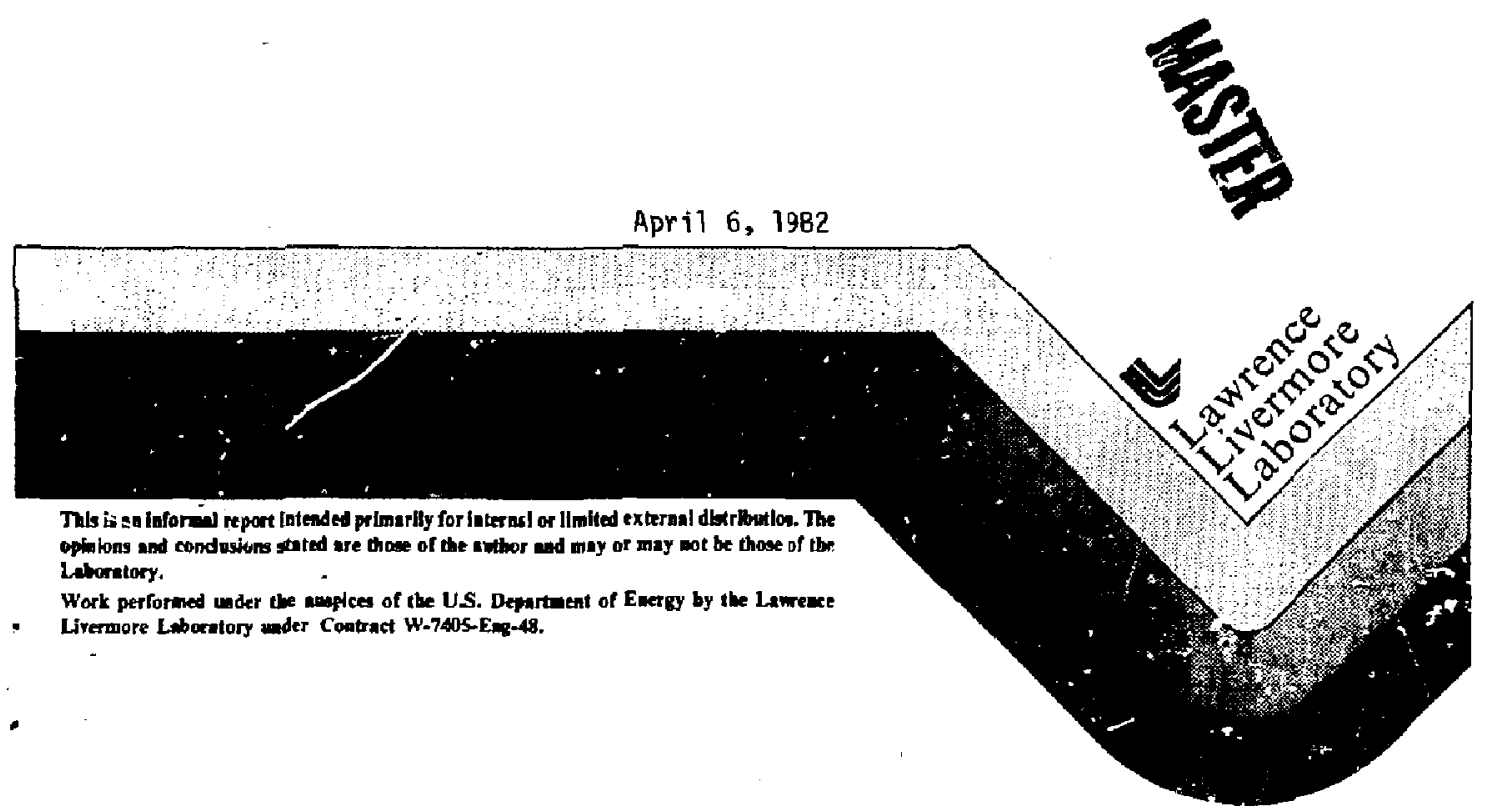

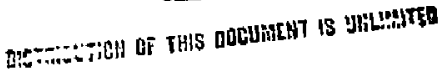




\section{NEGATIVE-POTENTIAL OPERATION OF TMX-UPGRADE}

P. Poulsen, S. L. Allen, D. E. Baldwin, T. A. Casper, F. H. Coensgen, D. P. Grubb, R. S. Hornady, W. M. Nevins, W. E. Nexsen, G. D. Porter, T. C. Simonen, and G. R. Smith Lawrence Livermore National Laboratory, University of California Livermore, CA 94550

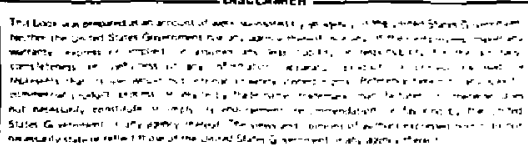

UCID--1934I

DE82 $01243 I$ 
Abstract 1

1. INTRODUCTION 3

1.1 Negative Tandem Mode of Operation 3

1.2 Historical Perspective 8

2. MODEL FOR COMPUTING THE AXIAL POTENTIAL PROFILES FOR NEGATIVE OPERATION 11

2.1 Description of the Model 11

2.1.1 Description of the electron densities: $n_{e j}(\varphi, \theta) \quad 12$

2.1.2 Description of the ion densities, $n_{i}(\varphi, B) \quad 16$

2.2 Method of Solution 17

2.3 Types of Solution 20

2.4 SUmmary

3. PUMPING OF NEGATIVE POTENTIAL WELL 37

4. FEED OF HOT-ELECTRON POPULATION 45

5. ECRH POWER REQUIREMENTS 63

5.1 Present Best Estimates of Required ECRH Power 63

5.2 Comparison of Theory With Previous ECRH Experiments j5

5.3 Discussions 68

6. MICROINSTABILITY 71

6.1 Theoretical Review 72

6.2 Experimental Review 78

6.3 Finite Geometry Stabilizing Effects on UHLC Instability 87

6.4 Stability Code 82

6.5 SUmmary 90

7. LOW-FREQUENCY STABILITY

8. RADIAL TRANSPORT IN THE CENTRAL CELL 93

$\begin{array}{ll}\text { 9. START-UP } & 101\end{array}$

9.1 Phase 1 - Establishing an MHD Anchor 106

G.1.1 Plasma initiaticn 107

9.1 .2 ECRH turnion 108

9.2 Phase 2 - Turning on the Central-Cell Neutral Beams 109

9.3 Fina? Phase - Pump Beam Turn-on and Evolution

to Equi]ibrium 
10. Operating Parameters

11. Diagnostics

\section{Appendices}

A. START-UP FORMULARY

A1. Hot (Mirror-Conf ined) Plug Electrons $\quad 126$

$\begin{array}{ll}\text { A1.1. Particle Batance } & 126\end{array}$

$\begin{array}{ll}\text { A1.2 Power Blanace } & 128\end{array}$

A2. Central-Cell Electrons 130

A2.1. Particle Balance 130

A2.2. Power Blanace 131

A3. Lentrai-Se]1 Hot (Mirrar-Sonfined) Ions 135

A3.1. Particle Balance 135

A3.2. Power Balance 137

A3.3. Quasi-Linear Stream Requirement 138

A4. Central-Cell Cold Ions 138

A4.1. Particle Balance 138

A4.2. Power Balance 140

A5. PTasma Potential 141

A5.1. Phases 1 and $2 \quad 141$

A5.1.1. Plug potential dip, $\Delta \varphi \quad 141$

A5.1.2. Central-cell potential, $\varphi_{e} \quad 141$

A5.2 Phase $3 \quad 142$

A5.2.1 Plug potential dip, $\varphi_{e} \quad 142$

A5.2.2 Central-cell potential, $\varphi_{i}$

B. CENTRAL-CELL PARTICLE AND POWER BALANCE IN NEGATIVE

POTENTIAL OPERATION 
SECTION 1

INTRODUCTION

\subsection{NEGATIVE TANDEM MODE OF OPERATION}

The tandem-mirror configuration is created by conbining hot-electron end-cell plasmas with neutral-beam pumping. The potential at the magnetic minimum of each end cell is sufficiently negative with respect to the central cell potential to confine central-cell electrons. The potential profile is shown in Fig. 1-1. Just inside the outer mirror point, the potentiai rises from its negative value to become positive with respect to the central cell. This greater positive potential serves to reflect ions escaping from the central cell ano thus provides the ion confinement. The ambipolar potential of the central cell is generally negative with respect to ground; hence the name "negative" tandem mirror. The negative potential well at the magnetic minimum of each end cell is maintained within the hot, magnetically-confined eiectron plasma by using charge-exchange neutral-beam pumping to remove ions. What are the advantages of this configuration over the positive tandem mirror with thermal barriers as exemplified by the proposed positive mode TMX and MFTF-B experiments? The primary advantage of this mode of operation is that it generates a tandem conf iguration without the use of an energetic, mirror-confined sloshing-ion population in each end ce11. The neutral beams that support the sloshing ions are eliminated with a rosulting reduction in circulating power and machine complexity. In a negative machine, the electroscatically confined end-cell ion populations are not suscejtible to either loss cone or anisotropy driven instabilities. Also, the pumping requirements do not appear to be as stringent as in a positive tandem machine; pumping is relatively more efficient since primarily trapped ions are pumped in a negative machine as opposed to the unavoidable pumping of both passing and sloshing ions in a positive machine. The selectivity of trapped-ion pumping is also an important issue for schemes using pumping by magnetic field perturbaticn.

A primary difficulty anticipated with the negative machine is the formation and maintenance of the hot-electron end-cell plasma at the desired density and temperature with the power available. Various electron microinstabilities may contribute to the loss of power, though hot-elertron 

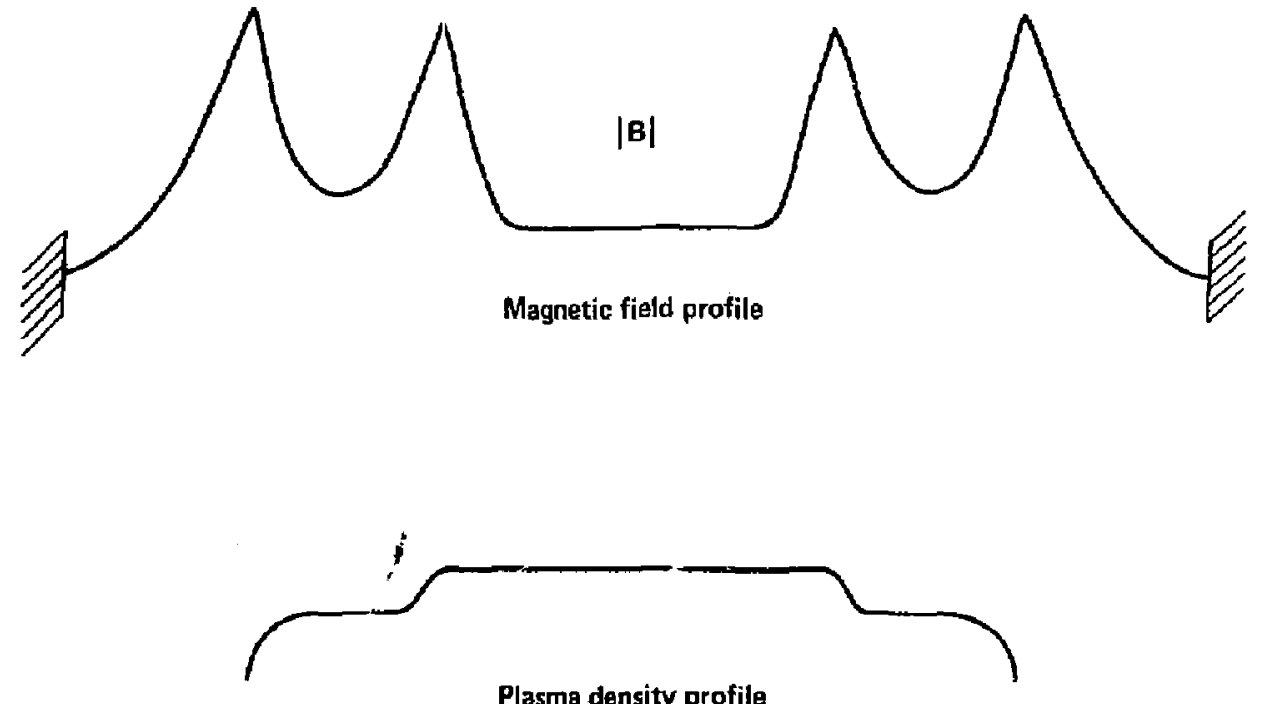

Plasma density profile

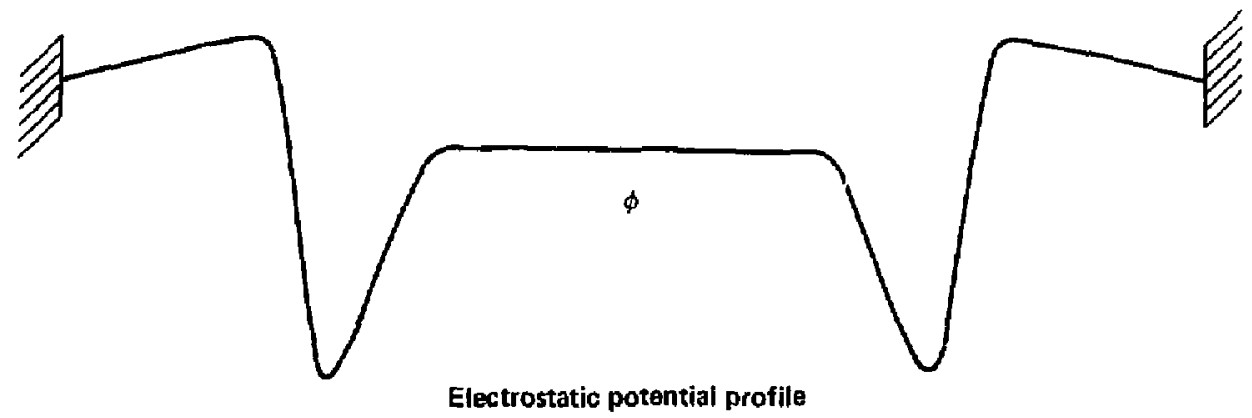

Fjgure 1-1. The negative tandem-mirror configuration. 
plasmas with densities near the required densities have been producod. Warm e lectron fueling of the hot-e lectron plasma must satisfy several criteria in order to avcid dissipation of the ECRH power in the fueling region and in order to produce a hot-electron population having suitable parameters.

A negative tanden lacks the stabilizing pressure in a good curvature region supplied by the sloshing ion population. Over a wide range of operating parameters, the hot electrons will not act as an MHD fluid and the pressure of the hot electrons will not coniribute directly to MHD stability. However, the pressure of warm end-cell ions and electrons in the radial magnet ic well enhanced by the hot electrons will provide MHD stability, as in the EBT experiments.

The central-cell potential is typically below ground potential; this affects the radial transport of both hydrogen and impurity ions. Initial estimates indicate inward radial transport due to neoclassical diffusion. It is therefore possible that the radial transport of the negative machine will be much less than for the corresponding positive tandem mirror configuration. This is an advantage for hydrogen ion confinement; a disadvantage of the inward radial transport is the possibility of enhanced impurity trapping from the edge plasma.

This report evaluates the possibility of operating TMX-Upgrade with negative potentials. We find that the physical changes to the machine are minor, involving primarily relocation of the microwave horns, and that it should be possible to generate a negative-potential tandem configuration having attractive confinement parameters $\left(\mathrm{n \tau}>10^{i ?} \mathrm{~cm}^{-3} \mathrm{~s}\right.$ ). Improved performance can be achieved by optimization of the magnetic field geometry.

A discussion of the evolution of the confinement concept shows how the negative-potential machine is related to simple and thermat-barrier tandem mirrors. Plasmas with negative potentials have been created previously, notably CIRCE ${ }^{3}$ and Table Top. 4 The new element added to produce highly-negative potential plasmas in the end cell is the enhanced removal of ions by pumping.

The method used to calculate the axial potential distribution is given in Section 2. The depth of the electron confining potential depends on the density of the energetic end-cell electrons relative to the central-ceil density, and is a function of the jon trapping and pumping rates. The sensitivity to these parameters is discussed. Adequate potentials for 
significant electrostatic conf inement of central-cel1 electrons are obtained over a wide and accessible range of machine operating parameters.

Removal of ions from the end-cell regions is accomplished by charge exchange of trapped ions with neutral beams aimed within the boundary for trapping of ions by the combined electrostatic and magnetic fields. The rate of trapping of the central-cell ions streaming thraugh the end cell must be countered by an equal rate of ion pumping. The amount of pumping also affects the depth of the electron confining potentials and hence the particle confinement. The results of a quantitative study of the pumping requirements are given in Section 3 .

The electrons required to build up and maintain the energetic end cell populations are trapped frori the center cell population by large ( $100 \mathrm{~V} / \mathrm{cm})$ microwave fields at the location of the fundamental electron cyclotron resonance. Certain conditions must be satisfied by the potential and magnetic configuration of the end cell so the electrons heated in the resonance region are trapped in the end cell. The energy of the electrons is limited by a resonance region of 1 imited spatial extent in order to 1 imit the electron pressure at the required density. These considerations are discussed in detail specific to the TMX geometry in Section 4.

The energetic electron population is subject to particle and power losses by the effects of instabilities and collisions. Section 5 reviews the power requirements for a number of hot-electron experiments and relates the observed power input to the classical scattering losses. It is found that the microwave power is great $1 y$ in excess of that required by a simple classical estimate; various ways of minimizing the power requirements for TMX are discussed.

The microstability characteristics of past hot-electron confinement experiments are reviewed in Section 6. A comparison with available theory shows good agreement between observed instabilities (Timofeev, U.H.L.C., and Whistler) and theoretical stability boundaries. Two experiments appeared to have good stability properties. A theoretical effort is under way to assess the effects of realistic velocity distributions and finite geometry on electron micro-instabilities. The methods and status of that work is discussed.

The contributions of the hot-electrons pressure to MHD stability is a subject of current investigations. The negative-potential configuration does not use a hot-ion population in the end cells, and the ion pressure is 
contributed only by the passing and trapped ions. Est imates of MHO stability limits are given in Section 7.

A major contributor to radial flow is the neoclassical transport caused by the noncancellation of drifts in a nonaxisymmetric central cell, as in TMX. Since the potential of the central cell is negative, this transport is inward, aiding in confinement of hydrogen, but also raising the possibility of enhanced impurity trapping from the edge plasma. These issues are the subject of Section 8.

Operation of the machine in the negative tandem mode can on $1 \mathrm{y}$ be accomplished if the hot-electron end-cell plasmas can be pumped; thus, the rate of scattering of the ions from the central cell into the low potential regions must be less than the available pumping rate. Since the scattering rates increase at low ion temperature, the pumping requirements at start-up may be excessive. It is therefore necessary to develop a scenario that allows the development of the negative tandem from a cold start-up plasma without excessive trapping. This scenario, involving start-up with a low-density beam-injected central-cell plasma with ECRH and neutral beam pumping in the end cells, is presented in Section 9. The equations employed to represent the plasma state and the particle and energy flow are given in the Appendices $A$ and $B$. One purpose of this work is to develop the relations that form a bas is for a future numerical study of the start-up process.

The steady state operating parameters for an axial $\mathrm{n \tau}=10^{12} \mathrm{~cm}^{-3} \mathrm{~s}$ are given in Section 10 . The calculations are done for the unmodified TMX-Upgrade magnetic-field configuration and several assumptions regarding the radial transport. Optimizing the magnetic configuration can subsiantially enhance the axial confinement.

The plasma diagnosic instiumentation for both negative and positive operation is similar, as discussed in Section 11. Two diagnostics, the heavy-ion beam probe and ion end-loss analyzer, can measure the plasma potential along the machine; Doppler broadening of impurity line emission yields information on the sense of the radial electric field. 


\subsection{HISTORICAL PERSPECTIVE}

Simple tandem-mirror machines confine plasma in a large volume central cell by creating a differance in potential between the central cell and small volume end cells. In $T H X,{ }^{5}$ it was shown that a mi, ror-confined ion population in each end cell produceo an end-cell potential that was positive with respect to the cential cell. Confinement of ions in the central cell was therefore electrostatically enhanced. The pissma potential of the central cell was positive in order to confine electrons equally'as well as ians and establish the ambipolar loss rate.

A simple negative machine is also possible, i.e., a machine in which end-cei] electrons rather than ions are mirror confinæd. This option has been considered earlier by Fowler. ${ }^{6}$ In this machine, the hot-electron density in the end cells e: ceeds the central-cell density. Since the lifetime of the end-cell electrons is determined by mirror confinement, the end-ce 11 p lasma develops a negative potential which enhances the ion lifetime by ambipolar potential confinement and thus maintains charge neutrality. It was found, however, that the potentia] difference between the end cell and central cell required to confine central-cell electrons demanded that the density difference between the central cell and end cell.s be lirge. As a consequence, the end-cell density was found to be excessive in terms of power loss and required microwave frequencies.

A later. development enabled the construction of a large, negative end-cell potential without employing hot-electron end-cell plasmas much denser than those in the central cell. Ciiculations showed that, if ions were removed (pumped) from the region of the mirror-confined hot electrons at a sufficient $r$ ate, a large negative potential could be produced even in end-cell plasmas having densities less than the central-cell density. ${ }^{7}$ The negative potential develops to maintain charge neutrality in the end cells. Thus, if the end-cell electron lifetime is enhanced by mirror confinement and ions are removed from the end cells by a pumping ircchanism, the loss of ions by scattering from this region is reduced by the formation of a deep potential well until the charge flows are equal. This mechanisin can be employed to create the thermal barrier for positive tandems and it can also be employed to create the eleccron confining potential of a negative tandem mirror. We compare the axial potential distributions of simple and pumped tanden mirrors 
in Fig. i-2. A simple positive tandem mirror is shown in Fig. 1-? (a) (as created in the original ThX experiment), a thermal-barrier tandem mirror in Fig. ]-2(b) [to be created in the TMX-Upgrade, positive mode which is referred to as TMX(P)], and a negative tandem mirror in Fig. 1-2(C) [referred to as $T M X\{N\}$, or the negative mode of operation of $i H X$-ijpgrade]. One method of pumping ions out of a potential weil consists of passing a beam of energetic neutra? atoms through the plasma. If the conditions for magnetic and potentia confinement are not satisfied by jons produced by charge exchange of the neutrals with potential-trapped ions, the net effect is the removal of trapped ions from the potential well. The neutrals that are produced by neutralization of the trapped ions escape the plasma across the field lines.

The density of the end cells in which the negative potentials are located can be substantially less than the density of the central cell. It is this feature that makes tandem mirrors attractive from the point of view of making a fusion reactor. The lower density of the hot-electror end-cel? plasma decreases the power and frequency requirement for the microwave heating. In the experimental design discussed below, the density of the hot-electron plasma is less than the central-cell plasma. Increasing the end-cell mirror rat io and decreasing the ratio of the thermal electron temperature to the ion temperature would further decrease the end-cell density and the ECRH power reouirement. 

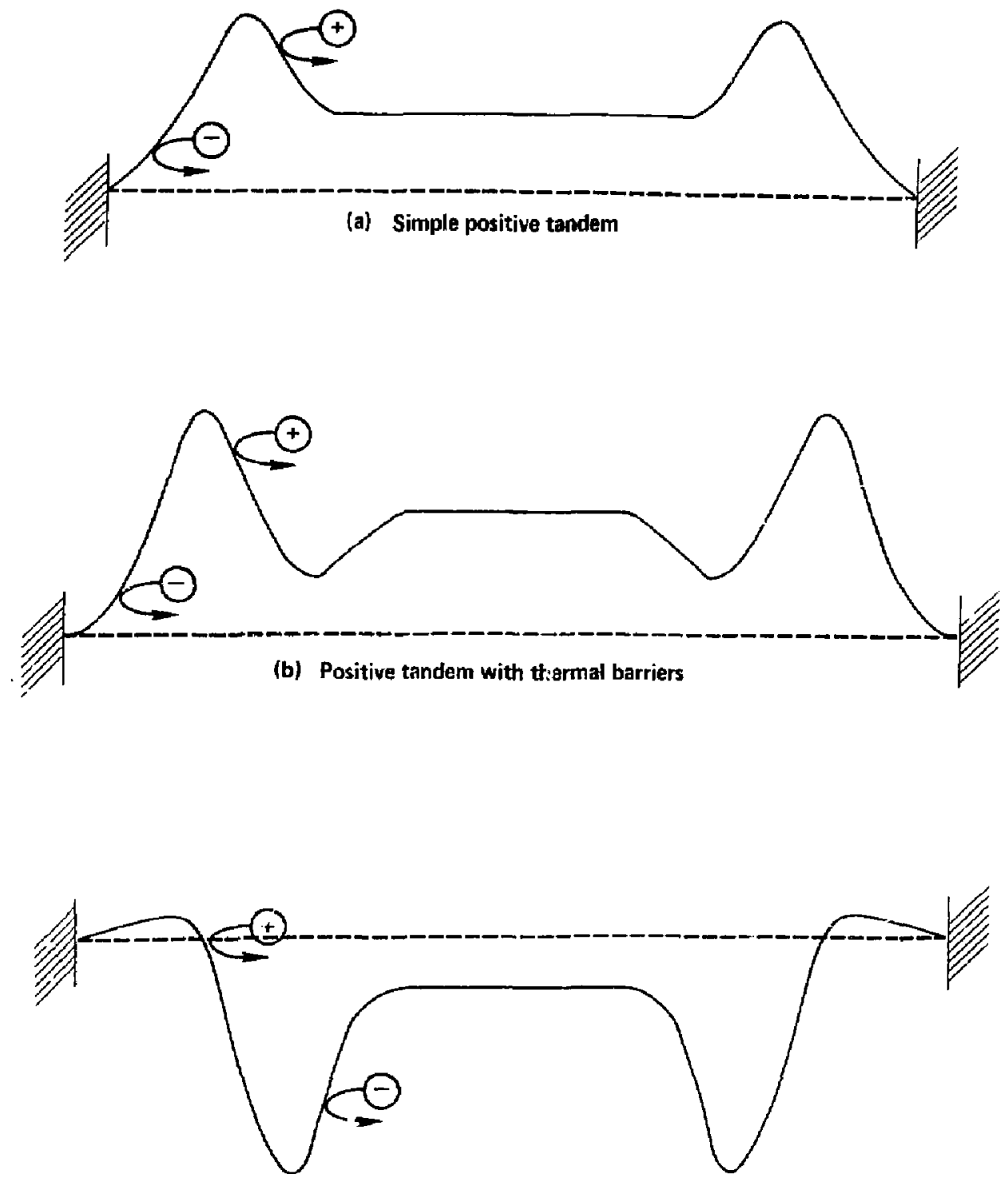

(c) Negative taijdem mirror

Figure 1-2. Axial potential profiles of tandern mirrors. 


\section{SECTION 2}

MODEL FOR COMPUTING THE AXIAL POTENTIAL PROFILES FOR NEGATIVE OPERATION

$$
\text { (S. L. Allen, G. D. Porter, P. Poulsen) }
$$

The most important key to the effective operation is the formation of the potential profile. This section describes an axial potential model for the plug region. The potential is calculated with respect to the central coil (more specifically, thi? inner (central-cell) mirror peak). A modei for the region between the outer mirror peak and the wall is under development.

The models for the electron and ian densities as a function of potentia and magnetic field are presented first. The assumptions and limitations of the model are also presented. This is followed by the results of a detailed parameter search, including changes in the magnetic field profile.

\subsection{DESCRIPTION OF THE MODEL}

The potential model is besed on the quasi-neutrality of the flasma, that is, any charge imbalance over a distance of roughly the Debye length will be cance?ed out by the collective behav or of the plasma coristituents. The Debye length is given by

$$
{ }{ }_{0}(\mathrm{~cm})=743 \mathrm{~T}^{1 / 2}(\mathrm{ev}) \mathrm{n}^{-1 / 2}\left(\mathrm{~cm}^{-3}\right),
$$

for the species with density $n$ and temperature $T$. Under most plasma conditions of interest, $\lambda_{D}$ is small $\left(\sim 10^{-2} \mathrm{~cm}\right.$ for $n_{e}=10^{12}$ and $\left.T_{e}=1000 \mathrm{eV}\right)$. The condition of quasi-neutrality requires:

$$
\sum_{i j} z_{i} n_{i j}(\varphi, B)-\sum_{j} n_{e j}(\varphi, B)=0
$$


where $Z_{j}$ is the charge on the ion and $j$ identifies the type of ion or electron species. Both the electron $\left(n_{e j}\right)$ and $j$ on $\left(n_{i j}\right)$ densities are functions of the electric potential $\varphi$ and the magnetic field $B$. The sums are carried out over ali the classes of particles that are present in the plasma. Solution of this equation in its general form requires a detailed knowledge of both the impurity behavior and the plasma behavior. Therefore, certain simplifying assumptions must be made to make the problem tructable. A careful delineation of these approximations must be made so that the validity of the solutions from the modsl can be judged under a specific set of conditions. First, it will be assumed that the impurity density is sufficiently small so that it does not affect the potential; that is, that $z_{i}=1$ for all classes of ions. Second, note that both the ion and electron densities are functions of $\varphi$ and $B$ which are in turn functions of 2 (the axial distance measurec from the central cell) and $r$ (the plasma radius). This madel will be limited to the calculation of the axial dependence of these quantities and a solution will be obtained at $r=0$ (the center-line of the plasma). Furthermore, it vill be assumed that the axial $\beta$-profile ( $\beta=$ ratio of kinetic to magnetic field pressure) does not significantly perturb the vacuum $8-f$ ield profile. This means that knowledge of $B(z)$ For the vacuum case $c$ an be used to transform a function of $B$ into a function of $z$ [for example, $\varphi(B)$ into $\varphi(z)]$. The final set of assumptions involves the analytic expressions that are used to describe the ion and electron densities. Because these are the most important part of the model, they will be considered in detail.

\subsubsection{Description of the Electron Densities, $n_{e j}(\varphi, B)$}

In the general case, the electron density may be made up of a large number of classes of particles based on their energies and types of confinertent (magnetically- or mirror-confined, potential-confined, or both, i.e., Yushmanov confined). For simplicity, only two distinguishabie populations of electrons will be used in the model: energetic, mirror-conf ined electrons (so-called "hot." electrons) and thermal electrons (so-called "cold" electrons). As will become more clear later, it is likety that the most serious error in the approximation is the treatment of the 
Yushmanov-trapped electrons which exist between the inner-mirror peak (central-cell side) and the bottom of the potential well.

The hot-electron population exists as a result of ECRH heating in the end-cell regions at the 5-kG and $10-k 6$ points (see Ref. 1 for more details). This population is necessary to create the potential well which confines electrons in the central-cell region. Perhaps the best way to model the hot-electron distribution function (and hence the density as a function of potential and magnetic field) would be to use results ${ }^{\top}$ from Fokker-Planck computer calculations. Because analytical fits to these results are not yet available, a simpler model has been chosen. This model (discussed in datail in Ref. 2) is made up of three parts:

7. The distribution function of hot electrons is assumed to be Maxwellian at $z=b$, the axial position of the end-cell midplane, and the distribution is mapped to another point in $z$ :

$n_{e h}(z)=n_{e h}(b) \frac{B(z)}{B(b)}\left[\frac{\bar{E}_{e h}}{\bar{E}_{e h}+\pi\left(\varphi_{e}+\varphi_{i}\right)}\right]^{1 / 2}$

where $n_{e h}$ is the hot electron density, $\bar{E}_{\text {eht }}$ is the mean perpendicular energy of the hot electrons $\left(\vec{E}_{\text {eh }}=k T_{1} / e\right.$ for bi-Maxwellianl, and $\varphi_{j}$ and $\varphi_{e}$ are the ion confining potential and the electron confining potential, respectively, measured relative to the central cell.

2. The factor of $\pi$, the result for a Maswellian distribution function, is then replaced by the parameter s to model the highly non-Maxwel $\mathrm{i}$ ian nature of the distribution function at $z=b$.

3. The magnetic field dependence is adjusted so that $n_{\text {eh }}(z)$ is described by a purely mirror-trapped distribution function when the square-root term is nearly one (valid when the electron energy is much greater than the electron and ion confining potentials):

$n_{e s i}(z)=n_{e h}(b)\left[\frac{B h-B(z)}{B_{h}-B(b)}\right]$, 
where $B_{h}$ measures the axial extent of the hot-electron plasma (see Ref. 2,3 ). Then, the hot-electron density as a function of $\varphi$ and B becomes (see Ref. 2):

$n_{e h}(z) \cong n_{e h}(b)\left(\frac{B_{h}-B(z)}{B_{h}-B(b)}\right)\left[\frac{\vec{E}_{e h}}{E_{e h}+s\left(\varphi_{e}+\varphi_{j}\right)}\right]^{l / ?}$.

For temperatures of interest, $\bar{E}_{\text {ehl }}$ may be $30 \mathrm{keV}$ or larger, and $\varphi_{\mathrm{e}}$ and $\varphi_{j}$ need to be several keV for efficient confinement. Therefore, the square-root term will be very nearly one and the distritution will be very close to fully mirror-trapped as in (Eq.) 3 . For the results presented here, Eq. (3) has been used for the hat-electron density with the restriction that $\bar{E}_{e h L} \gg s\left(\varphi_{e}+\varphi_{j}\right)$. investigation of the sensitivity to the s-parameter is currently underway.

The other class of electrons is the cold or thermal population. These particles are primarily streaming electrons from the central cell and have a Maxwellian energy distribution with a temperature $T_{e c}$ and density $n_{c}$ in the central cell. As these particles stream inte the plug and experience the potential kell (hill for electrons) they must have an energy $E-\varphi(z)$ to reach the axial point $z$ ( $\varphi$ is measured relative to the central cell and $E$ is the total electron energy). Figure 2-1(a) shows a schematic plot or the axial magnetic field profile and the idealized (qualitatively correct) potential profile. A simple model has been used to specify the axial dependence of the thermal electron density:

$$
\begin{array}{ll}
n_{e c}(z)=n_{c} \exp \left(-\exp / k T_{e c}\right), & z \leq b\left(B=B_{p} \text { and } z=b\right) \\
n_{e c}(z)=0 & , z>b .
\end{array}
$$

To be sure, this model is not absolutely correct near $z=b$ (a truncated Maxwellian, for example, might be more appropriate). However, for interesting values of $\varphi_{e} / T_{e c}$, the density at this point is very small--about $0.3 \%$ of $n_{c}$ for $\varphi_{\mathrm{e}} / T_{\mathrm{ec}}=6$. Therefore, the details of the distribution function can be neglerted near the bottom of the poiential well. On the outboard side of the well, the electrons are accelerated and therefore have an even lower density. Thus, the thermal electron density is set to zero in this region. 

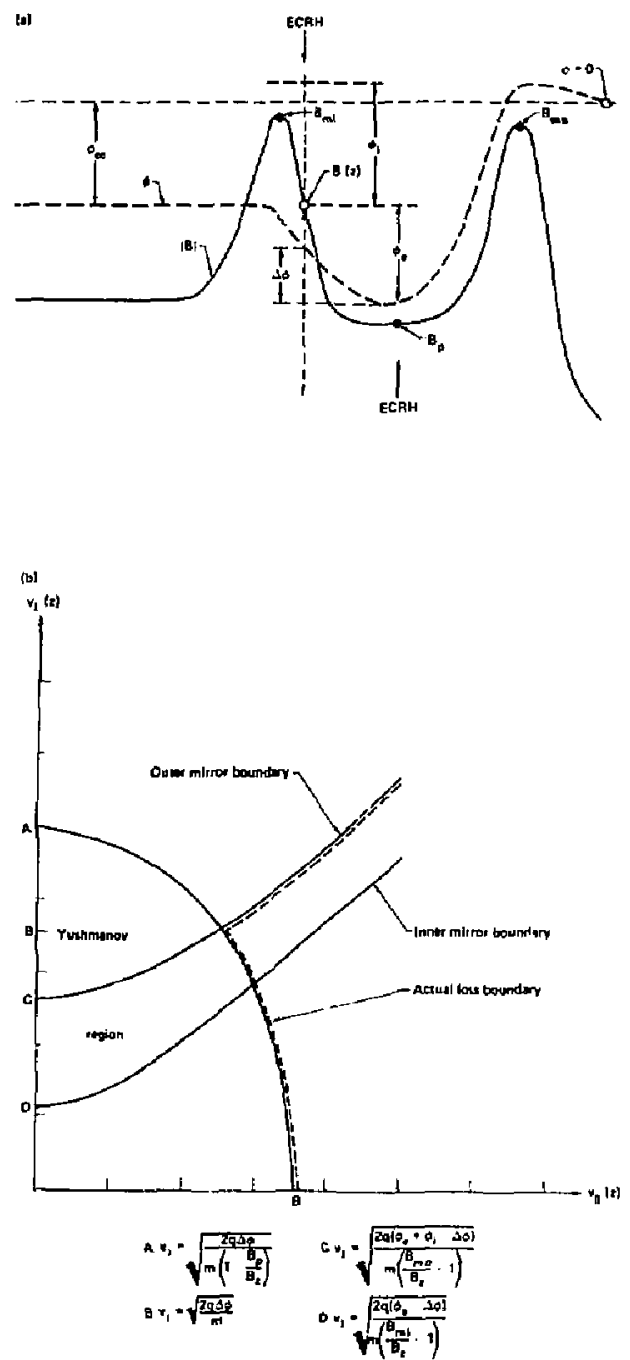

Figure 2-1. (a) Schematic plot of the axial magnetic field profile and the patential for the negative mode of operation. (b) Phase space plot at the ECRH rasonance point showing the confinement boundaries. 
Perhaps the most serious imitation to the model is the treatment of the Yushmanov-trapped particles as having a Maxwellian distribution. Fokker-planck calculations of an ECRH-heated plasma (Ref. 1) show that the actual distributions may be heavily weighted in the perpendicular direction, so the present model probabiy underestimates this portion of the electron density. As soon as usable analytical representations to these fokker-Planck resuits are available, they $y 11$ be incorporated in the model distributions of the electron densities. [Refer to $F i g .2-1(b)$ witich shows the velocity space boundaries near the ECRH resonance point.]

\subsubsection{Description of the Ion Densities $n_{j}(\varphi, \underline{B})$}

The ians are also modeled by two distinguishable classes: passing and trapped. The passing ions "pass" through the central cell and end cells since they flow through the inner mirror peak. They are reflected by the oart of the potential which is positive with respect to the central cell. The ions confined to the end cells are "trapped" ions and do not have sufficient energy to escape the negative potential well; these particles originate both by collisions of passing ions with other passing ions and by collisions between passing ions and trapped ions. The trapped-ion population can be actively modified by charge exchange pumping using energetic neutral beams. In this process, the collision of a trapped-ion and an energetic-neutral results in a low energy neutral which is not confined and leaves the plasma, and an energetic ion which is not confined by the potential and becomes a passing ion or leaves the plasma.

A Fokker-planck calculation of the trapping rate has been performed by LoDestro and is described in in Refs. 4 and 5 . In turn, these results were Fit by an integrable analytical form by $R$. Cohen. As described in Ref. 6 , the passing ion distribution function is,

$$
f_{p}=n_{c}\left(\frac{m_{i}}{2 \pi k T_{i}}\right)^{3 / 2} \exp \left(-\frac{E+E \varphi}{k T_{i}}\right),
$$

and the model distribution function for the trapped ions is,

$$
t_{t}=n_{c}\left(\frac{m_{i}}{27 T_{i}}\right)^{3 / 2} \exp \left[\frac{E+e \varphi-A u}{\left(a-1 / T_{i}\right.}\right]
$$


where $\mu \equiv R m_{i} V_{1}^{2} / 2, R(z) \equiv B_{m i} / B(z)$, and $A \equiv$ a filling parameter for the trapped ions. The limits of $A$ are:

$$
\begin{aligned}
& A \equiv 1 \text {, no trapped ions (inf inite pumping) } \\
& A+\infty,-\infty \rightarrow 0 \text {, increasing density of trapped particles } \\
& A=0 \text {, no pumping (Maxwellian trapped particles). }
\end{aligned}
$$

$R$. Cohen has integrated the velocity distribution to obtain the trapped and passing ion densities, $n_{i t}$ and $n_{i p}$ respectively, at given values of the potential $\varphi$ and the magnet ic field $B$. The equations taken from Ref. 4 are:

$\overline{A R-\ln i t}= \begin{cases}\left(\frac{\left.R-\frac{1}{R}\right)^{1 / 2} \ln n_{0}}{R}\right)^{1 / 2} \exp \psi & , R \geq 1, \phi \geq 0(9) \\ \left.2 \pi^{-1 / 2}\left[(A-1)^{1 / 2} D\left(x^{1 / 2}\right)-\left(\frac{l-R}{R}\right)^{1 / 2} D(1 \psi)^{1 / 2}\right)\right], & R<1, \phi<0(10) \\ 0 \quad & R<1, \phi \geq 0(11)\end{cases}$

$\frac{n_{i p}}{n_{0}}= \begin{cases}\exp \phi \operatorname{erfc}\left(\Phi^{1 / 2}\right)-\left(\frac{R-1}{R}\right)^{1 / 2} \exp \psi \operatorname{erfc}\left(\psi^{1 / 2}\right), R \geq 1, \phi<0(12) \\ \exp \phi\left(\frac{R-1}{R}\right)^{i / 2} \exp \psi & , R \geq 1, \phi>0(13) \\ \exp \phi \operatorname{erfc}\left(\Phi^{1 / 2}\right)+2\left(\frac{1-R}{\pi R}\right)^{1 / 2} D\left(I \psi 1^{1 / 2}\right) & , R<1, \phi=0 \text { (14) } \\ \exp \phi & , R>1, \phi \geq 0 \text { (15) }\end{cases}$

where $D(x)=\exp \left(-x^{2}\right) \int_{0}^{x} \exp \left(t^{2}\right) d t$ (Dawson's Integrai), $\phi=-\operatorname{e\varphi } / k T_{i}$, $\psi=R \Phi /(R-1), x=\Phi /(A-1)$, erfc $\{x)=2 \sqrt{\pi} 5_{x}^{\infty} \exp \left(-t^{2}\right) d t=1-\operatorname{erf}(x)$ is the complementery error function and $\operatorname{erf}(x)$ is the error function, and $n_{0}=$ the ciensity at the mirror throat.

\subsection{METHDD OF SOLUTION}

The four populations discussed above are substituted into the quasi-neutrality condition, Eq. (2), to obtain: 


$$
F(\varphi, B) \equiv n_{i p}(\varphi, B)+n_{i t}(\varphi, B)-n_{e h}(B)-n_{e c}(\varphi)=0 .
$$

A computer code is used to determine under what conditions there are solutions to this equation; the computational method is quite simple. Tha code uses two regions, $z\left(B_{m i}\right) \leq z<b$ and $b<z \leq z\left(B_{m o}\right)$ where $B_{m i}, B_{m o}$ are the inner and outer mirror fields, respectively. A value for the potential is selected and $F\left(\varphi, B_{m i}\right)$ and $F\left(\varphi, B_{p}\right)$ are computed to see if there is a sign change and hence a root exists in the first interval $\left(B_{p}<B<B_{m i}\right)$. If a root exists, a Newton-Raphson method is used to calculate the value of $B$ that satisfies Eq. (16) to a specified tolerance. The previous root (or $B_{p}$ if this is the first solution) is used for the first approximation, and the new approximation is given by,

$$
B_{i+1}=B_{i}-\frac{F\left(B_{i}\right)}{F^{\prime}\left(B_{i}\right)} \text {, fixed } \varphi \text { and } F^{\prime}=\partial F / \partial B \text {, }
$$

which is numeric 1 lly approximated by,

$$
B_{i+1}=B_{i}-\delta_{i}\left[\frac{F\left(B_{i}+\delta\right)}{F\left(B_{i}\right)}-1\right]^{-1}
$$

where $B_{i}$ is the initial (previous) solution, $F(B)$ is defined in Eq. (16), and $\delta_{i}$ is a small number $\left(<10^{-6}\right)$. If no root exists, the same procedure is carried out for the other interval. Values of $\phi / T$ ec from approximately -50.0 to +10.0 are normally examined in the code for possible roats.

This set of computed vaiues of $\varphi(B), n_{i p}(B), n_{i t}(B), n_{e h}(B)$, and $n_{e c}(B)$ are then converted to functions of 2 by knowledge of the axjal (vacuum) magnetic field profile. The code uses the output from the EFFI computer code (the EFFI code calculates magnetic field prcfiles from the input coil shapes and coil currents). As will be described later, changes in magnetic field profiles induced by varying (or turning off) certain coil currents can be used to tailor the axial potential profiles.

The types of solutions that are found under various input plasma conditions will be discussed in detail in the next section. In addition, a parameter survey will be presented. First, however, it is important to establish that this model does show that solutions with large negative potentials $\left(\varphi_{\mathrm{e}} / T_{\mathrm{ec}}\right.$ at least 6$)$ exist. One such example is shown in Fig. 2-2. F jgure 2-2(a) shows schematically the magnetic field profile (i.e., the inner 

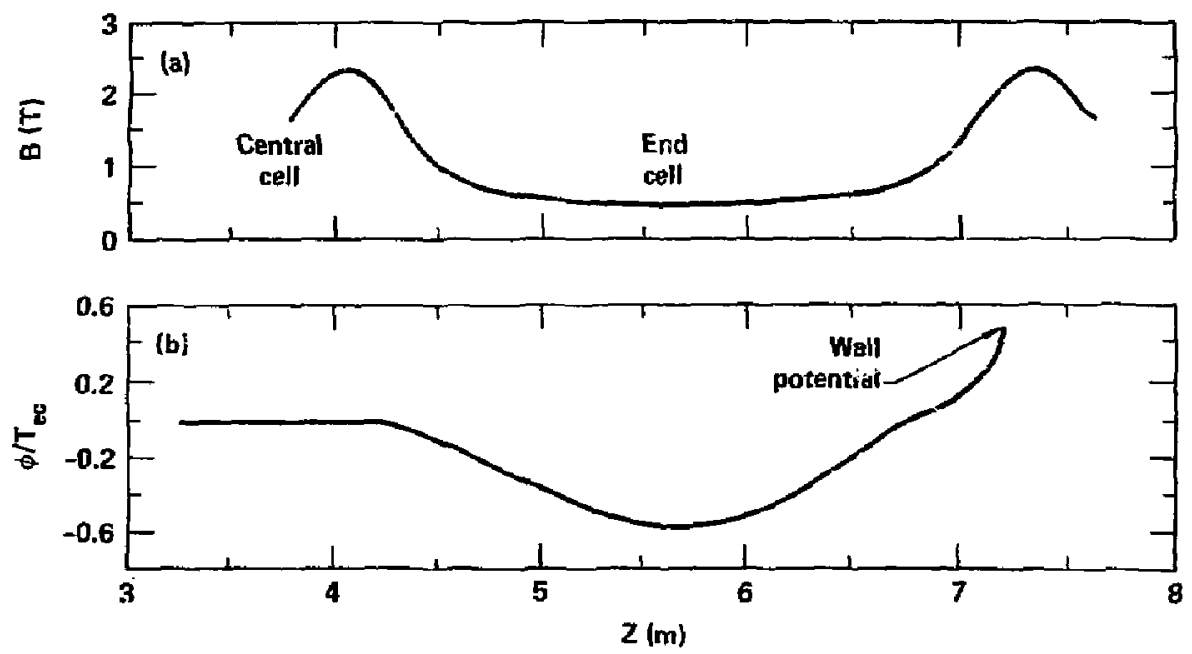

Figure 2-2 The magnetic field profile (a) and a calculated potential profile (b) for a case where $\varphi_{\mathrm{e}} / T_{\mathrm{ec}}$ is about 6 . 
and outer mirrors are bilanced, which is not true for TMX-N) and potential as a function of $z$. Figure $2-3$ shows $n_{e h}$ in (a) and $n_{e c}$ in (b), and Fig. 2-4 shows $n_{i p}$ in (a) and $n_{i t}$ in (b) for these conditions. Figurt 2-5 shows the axial profile of the total particle density in the end cell. The potential well has a value of approximately $\varphi_{e} / T_{e c} \sim-6$ in this case.

\subsection{TYPES OF SOLUTIONS}

The computer model has been used to investigate the types of solutions that are obtained with a given set of input parameters. These parameters are described in the code by the variables:

A - controls the trapped-ion distribution function

Tedei - the ratio of the electron temperature $T_{e c}$ to the ion energy $\mathrm{E}_{i}\left(3 / 2 \mathrm{kT} \mathrm{T}_{i}\right)$

Rnhnc - the ratio of the hot-electron density at the midplane of the plug to the Maxwellian electron component of the central-cell density

Bh - the axial extent of the hot-erectron distribution

$B$ mi - the magnetic field at the inner (central-cell side) mirror

$B p \quad$ - the magnetic fie!d at the midp?ane of the plug (also the minimum of the potential).

The last two quantities are determined by the magnetic geometry and therefore are fixed for a particular coil set and magnet current. The $i$ $t$ three parameters are a result of neutral beam pumping ( $A)$, ECRH heating (Rnhnc), and heat flow or supplemental heating by neutral beams or ICRH heating (Tedei and to some extent Rnhnc). The most general result of varying these parameters is that both adequate pumping and sufficient hot electron density are required for formation of useful potentiat welis . Figure 2-6 presents some of the possible solutions as a function of the trapped electron density for the field profile shown in $F i g \cdot 2-6(a)$. As seen in Fig. 2-6(b) (and first identified ky $G$. Porter), if $n_{j t}$ is too large, the solution is double valued. Furthermore, this discontinuity develops at a value of about $\varphi_{\mathrm{e}} / \mathrm{T}_{\mathrm{ec}} \sim-4$ and is insensitive to changes in other parameters because there is a logarithmic dependence of the potential on these variables. As shown in Fig. 2-6i(c), a discontinuity in the solution (not necessarily occurring at the midplane of the plug but usually centered around the 


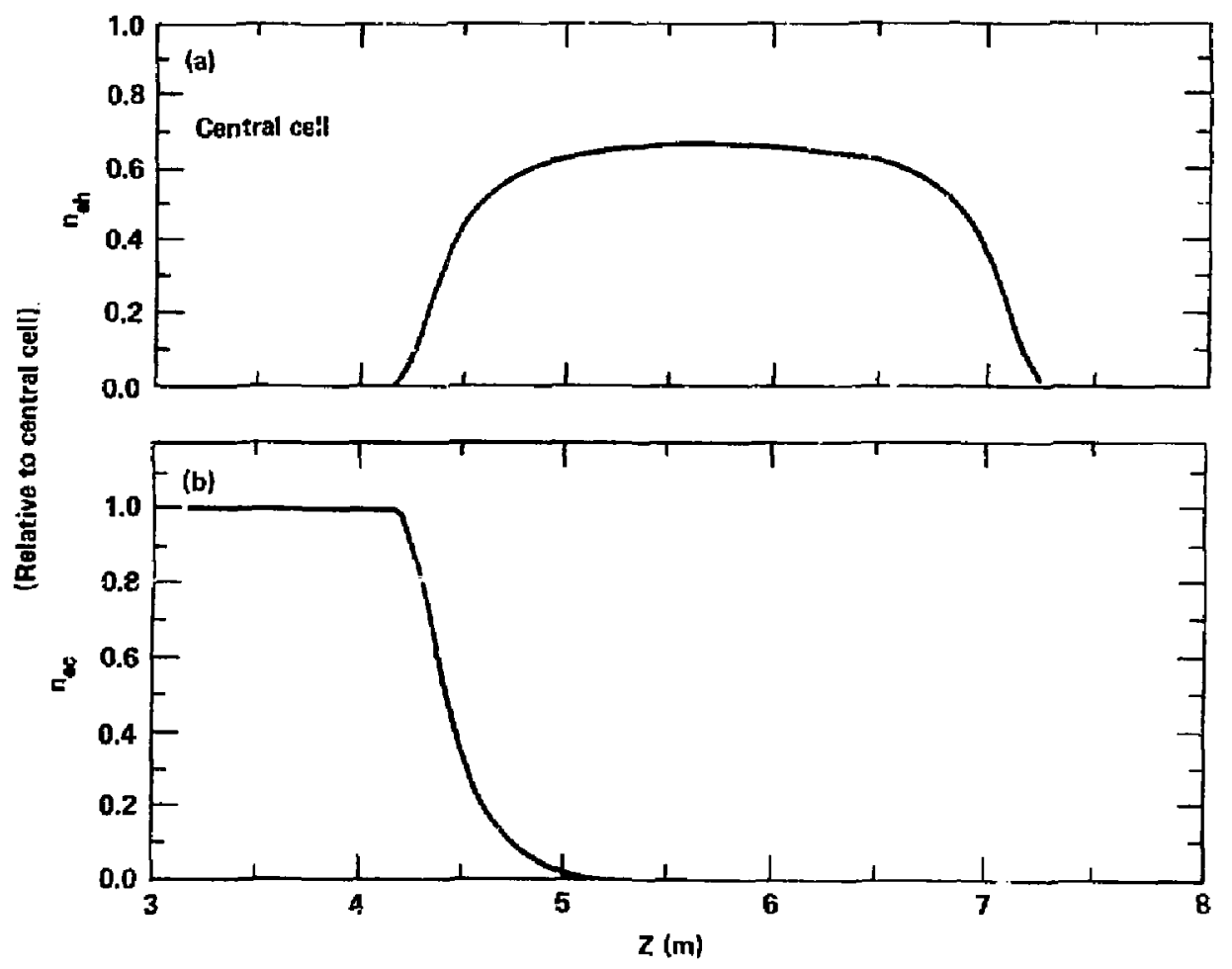

Figure 2-3. The axial hot-electron density profile (a) and the cold-electron density (b) for the potential profile shown in Fig. 2-2(b). Note that the cold-electron density is normalized to the central cell density. 


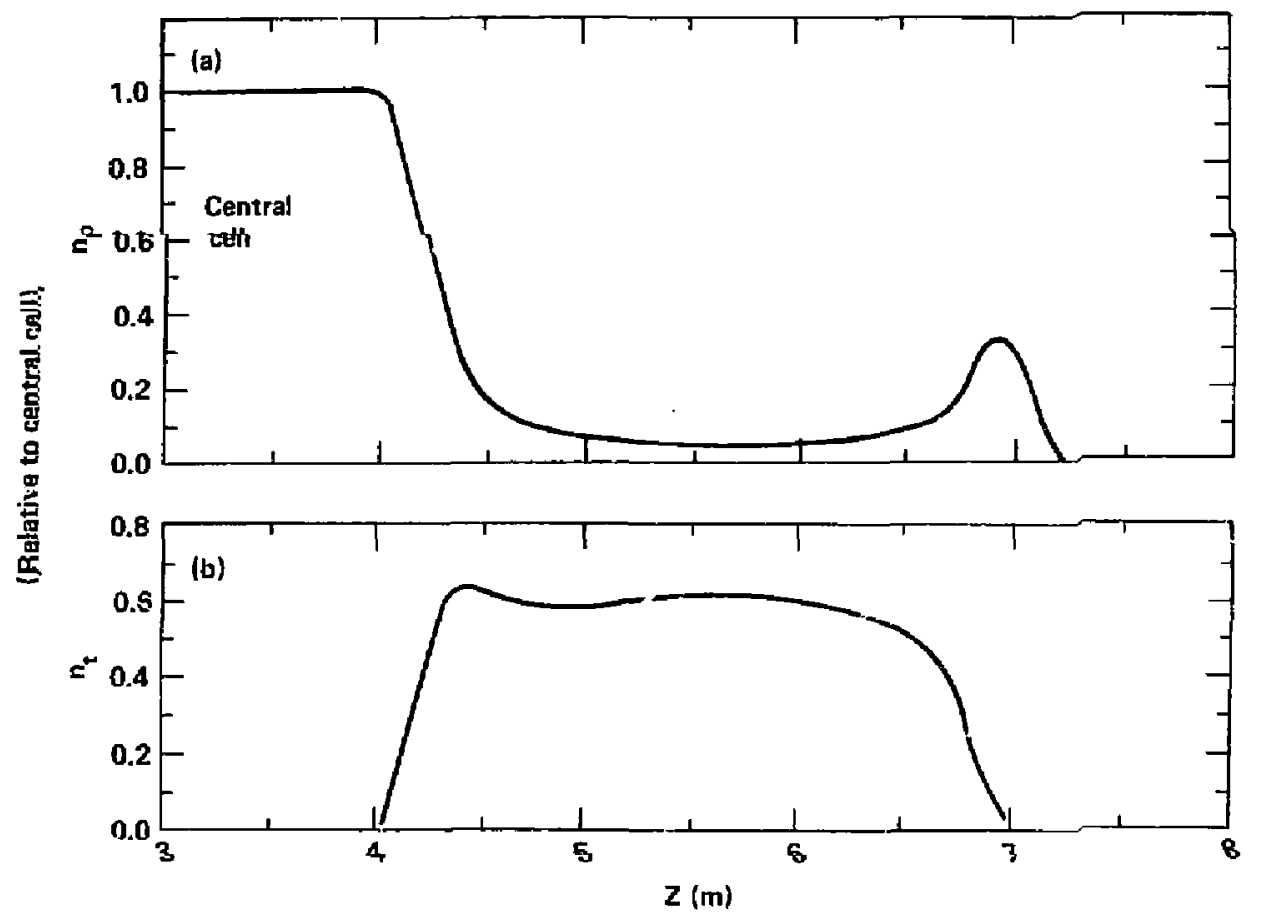

Figure 2-4. The axial profiles of the passing-ion density (a) and the trapped-ion density (b) for the potential profile shown in Fig. 2-2(b). The passing-ion density is referenced to the central cell. 


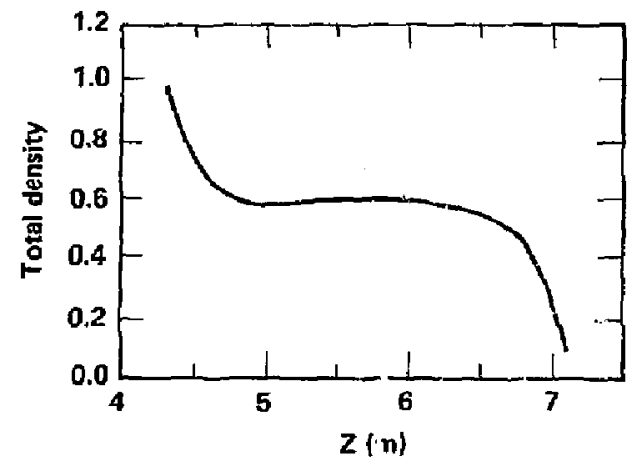

Figure 2-5. The axial profile of the total density (ion or electron) for the potential profile of Fig. 2-2(b). 


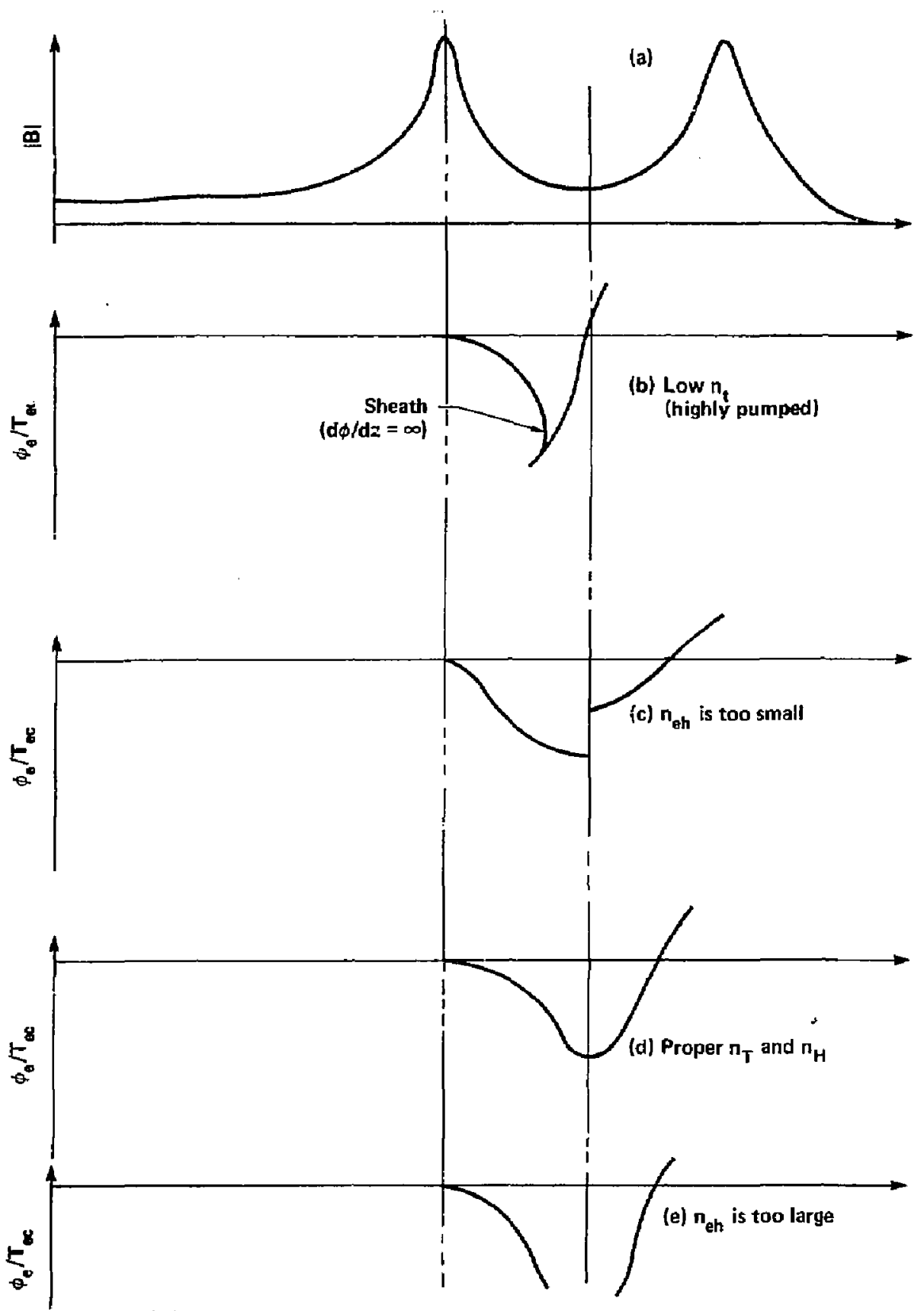

Figure 2-6. (a) A schematic representation of the axial magnetic field profile. (b) The axial potential profile when $n_{j t}$ is too luw (hinhly pumped); a roublevalued solution results. (c) The axial poteritial profile when the density of not electrons neh is too snall; a discontinuous solution results. (d) A continuous potential profile which results for the proper $n_{i t}$ and $n_{p h}$. (e) A discontinuous potential profile; the result of $n_{\text {eh }}$ to large. 
midplane) can occur if the density of hot electrons is too low. A case with the "proper" $n_{i t}$ and $n_{e h}$ is shown in Fig. $2-6(d)$, and this results in a continuous potential profile. Fig. 2-6(e) shows there is a discontinuity in the potential when $n_{e h}$ is too large. The exact parameter range for this condition is somewhat subjective, as it depends on the parameter "s" in Eq. (4) and also where the cutoff for the solution is selected.

These results are summarized in Fig. 2-7; the boundaries for these types of solutions are shown on a graph of Rnhnc $\left(n_{\mathrm{eh}} / n_{\mathrm{ec}}\right)$ versus $A$ for Tedei $\left(T_{\mathrm{ed}} / \mathrm{E}_{j}\right)$ of $0.5,1.0,1.25$, and, .5 . (Note that the dependence of $\mathrm{n}_{i t}$ on $A$ is not linear). Above each line and to the right of about $A=20$ is a region where the potential profile is continuous and $\varphi_{\mathrm{e}} / \mathrm{T}_{\mathrm{ec}}>-6$; this is the region of useful solutions. As mentioned above, the upper boundary of this region is somewhat subjective. A more detailed look at $g(b)=1+\left[n_{i t}(b) / n_{i p}(b)\right]$ versus $A$ for Tede $i\left(T_{e c} / E_{i}\right)$ of 0.5 and 1.0 . (see Fig. 2-8). It is very important to note that $\varphi_{\mathrm{e}} / \mathrm{T}_{\mathrm{ec}}$ has been held constant (about 6) for this graph. In general, $g(b)$ can either increase or decrease with $A$ depending on whether the potential is increasing or decreasing. A detailed parameter study is being carried out with the computer code to carefully investigate these trends.

As mentioned above, one of the most important parameters in this model is the trapped-ion distribution function which determines the density $\mathbf{n}_{\mathrm{it}}$. To be sure, the distribution functions used in the code are models and do not fully represent the physical situation. In a real experiment, the beam pymping will be spatially selective, so the pumping rate will be a function of the axial and radial coordinates. A background of neutral particles can also influence these distribution functions. These processes are currently being modeled and their importance will be evaluated. A detailed discussion of the pumping and validit; of the trapped-ion distribution function will be presented in Section 3 .

Another important parameter is the ratio of the hot-electron density to the Maxwellian component of the central-cell density Rninc $\left(n_{e h} / n_{e c}\right)$. It is desirable to minimize $n_{e h}$ as this parameter is determined primarily by the ECRH power input and its efficiency. On the other hand, $n_{e c}$ should be as large as possible to maximize the (fusion) power output of the central cell. The dependence of Rnhnc of the other parameters has been roughly est imated by examining the case of $g(b)=1$ (fully pumped) and $\varphi_{\mathrm{e}} / T_{\mathrm{ec}} \gg 1$ : 


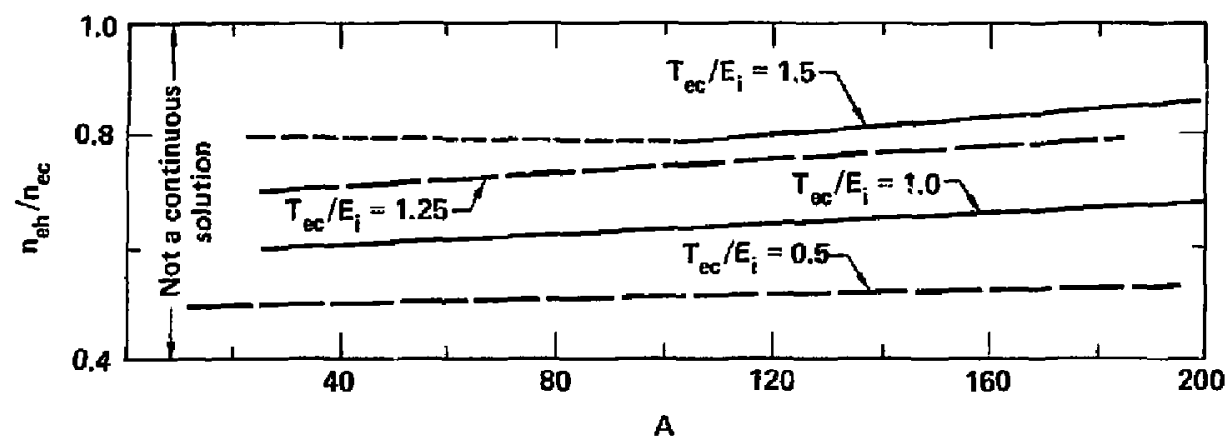

A

Figure 2-7. A plot of the ratio of $\mathrm{n}_{\mathrm{eh}} / \mathrm{n}_{\mathrm{ec}}$ vs. A at various values of $T_{e^{\prime}} E_{i}$. The potential $\varphi_{\mathrm{e}} / T_{\mathrm{ec}}$ is Tess than $\mathrm{b}$ below each line. The region where the potential becomes discont inuous [Fig. 2-6(e)] is near the top of the graph. The region where $A$ is small also results in a discontinuous solution. 


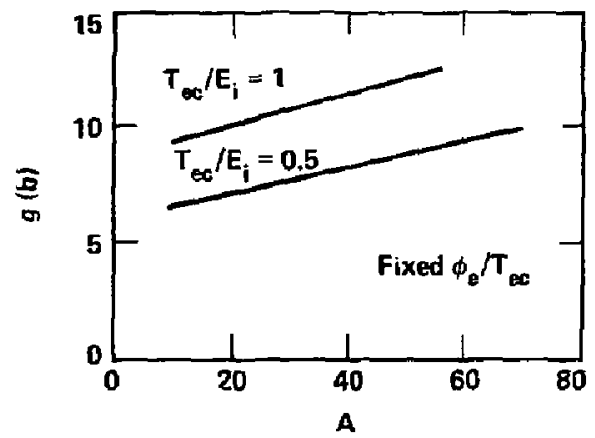

Figure 2-8. A plot of $g(b)$ - (the quantity $1+n_{i t} / n_{j p}$ evaluated a $z=b$, the bottom of the poteritial we11) - as a function of $A$ for various $\tau_{\mathrm{ec}} / \mathrm{E}_{j}$. Note that the potential $\varphi_{\mathrm{e}} / T_{\mathrm{ec}}$ is fixed in this plot. 


$$
\text { Rnhnc }=\frac{n_{\mathrm{eh}}(\mathrm{b})}{n_{\mathrm{ec}}} \simeq\left(\frac{1-\mathrm{B}_{\mathrm{p}}}{\mathrm{R}_{\mathrm{m}}}\right)^{1 / 2}\left(\frac{\mathrm{T}_{\mathrm{ec}}}{T_{\mathrm{i}}}\right)^{1 / 2}\left(\frac{\varphi_{\mathrm{e}}}{\mathrm{ec}}\right)^{1 / 2} \text {. }
$$

where $R_{m}$ is the mirror ratio, $\beta_{p}$ is the plug beta, and $T_{e c}, T_{i}, \varphi_{e}$ are defired as above. Preliminary results isdicate that this scaling is approximately correct for other values of $g(b)$. Therefore, for fixed $n_{\mathrm{ec}}$, $B_{p}, T_{e c} / T_{i}$, and $\varphi_{e} / T_{e c}$ it should be possible to decrease the required ECRH power by changing only $R_{m}$, the mirror ratio between the :nner (central cell) magnetic-field peak and the midplane of the plug.

The dependence of the potential profile on $R_{m}$ (and thereby Rnhnc) was tested for the TMX(N) magnet geometry. As mentioned previous]y, the computer code accepts the output of the EFFI magnetic-field calculation code. (Recall that the EFFI code uses models of the actual magnet configurations and currents to compute the vacuum magnet ic field profile.) Three cases were zested:

1. The "normal" magnetic field profile.

2. The current in the inner (central-cell side) solenoid coil around the Ioffe set (the so-called "inner belly-band" - see Fig. 2-9) was turned off.

3. The current in both "belly bands" was turned off.

The magnetic field and potential profile for case 1 are shown in Figs. $2-10(a)$ and 2-10(b), respectively. The parameters of $A=2000$, Tede $i=0.75$, and finhnc $=0.7$ resulted in a potential of $\varphi_{\mathrm{e}} / \mathrm{T}_{\mathrm{ec}}$ of about -10 . The mirror ratio in this case is about $2.2 / 0.5=4.4$. Similar plots for case 2 are presented in Fig. $2-11 ; R_{\mathrm{m}}$ is about $2.15 / 0.35=6$ and $\varphi_{\mathrm{e}} / T_{\mathrm{ec}}-22$. Figure 2-12 shows the results for case $3 ; R_{m}$ is about $2.15 / 0.2=10.8$ and the potential is quite large. It should be noted that the exact value of the potential in this case is not correct as the parameter $s$ was set to zero far simplicity and $E_{e h 1}$ is probably not much greater than $\varphi_{e}$ [see Eq. (4)]. Nevertheless, the potential is much larger than in case 1.

In order to test the $\mathrm{R}_{\mathrm{m}}$ dependence in $\mathrm{Eq}$. (18), Rnhnc was next decreased in case 3 until it had the same $\varphi_{e} / T_{\text {ec }}$ as case 1 . As shown in Fig. 2-13, $R_{\text {. }}$ nc $=0.3$ resulted in $\varphi_{\mathrm{e}} / \mathrm{T}_{\mathrm{ec}}--10$. The mirror ratio in case 3 compared to case 1 is $10.8 / 4 . A \sim 2.45$ and the ratio of Rnhnc in case 3 to 


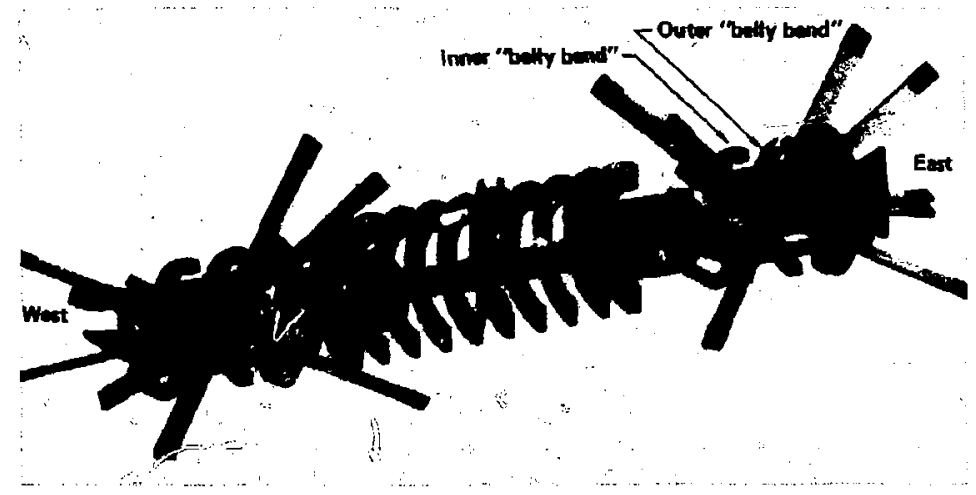

Figure 2-9. TMX-U magnet structure showing the location of the coils called the inner and outer "belly bands." 

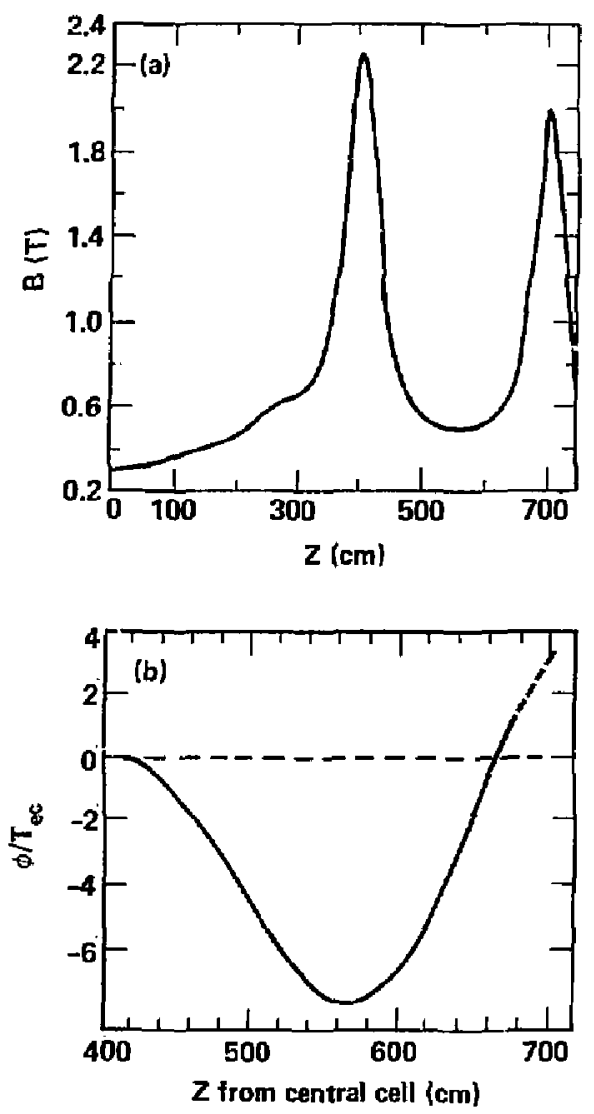

Figure 2-10. The normal TMX-U axial magnetic field profile (a) and a typical potential profile (b) for this case. 

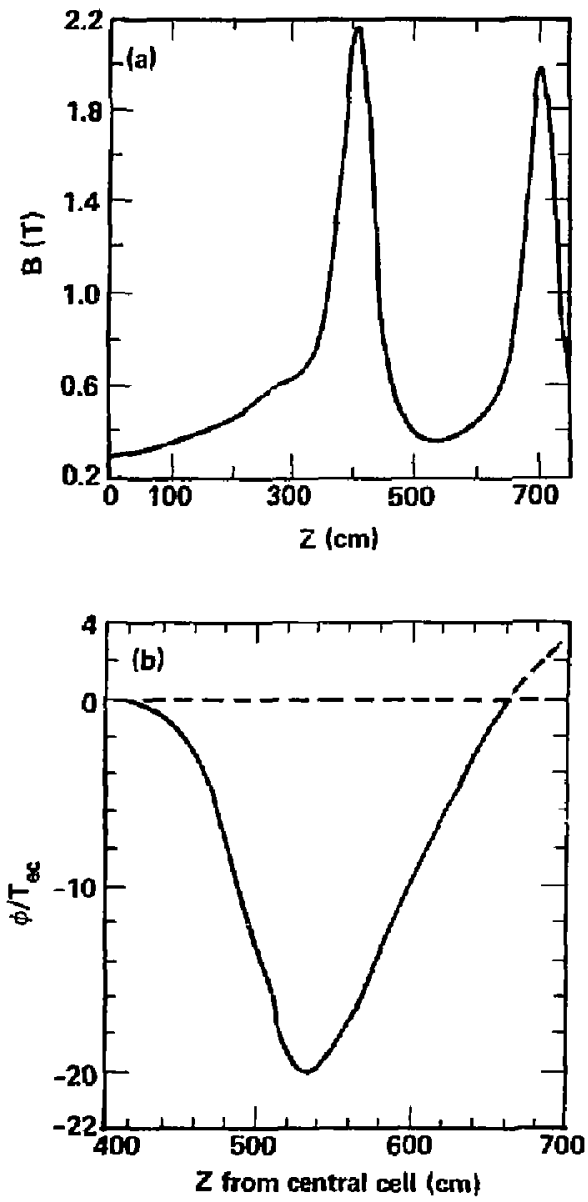

Figure 2-11. The axial magnetic field profile (a) for the case where the inner "belly band" coil has been turned off. The axial potential profile (b) for this case [a1] other parameters are the same as in Fig. 2-10(b)] shows a deeper potential well. 

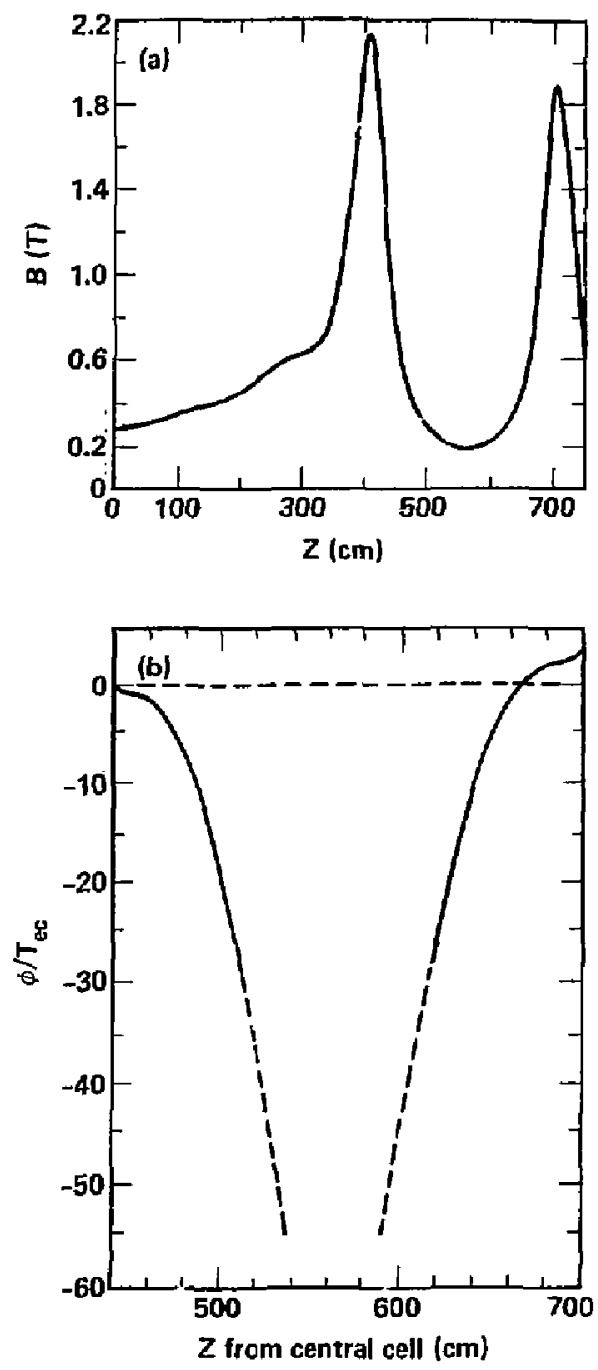

Figure 2-12. The axial magnetic field profile (a) for the case where both "belly band" coils have been turned off. The axial potential profile for this case [al] other parametrs are the same as in Fig. 2-10(b) and 2-11(b)] shows a very deep well. 


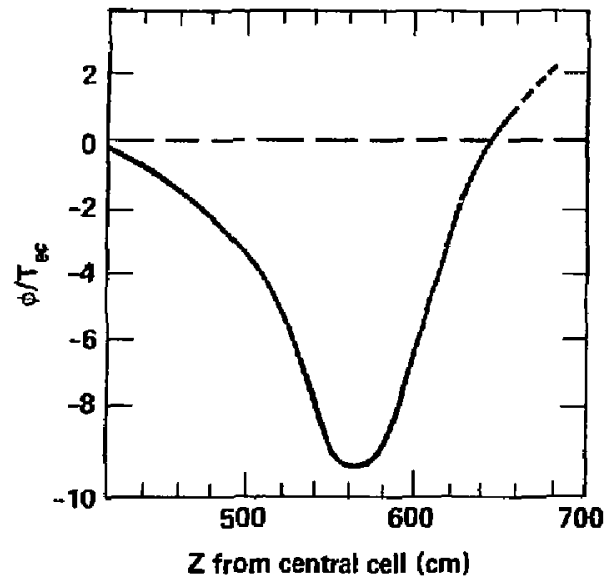

$F^{*}$ ure 2-13. The axial potential profile for the case where both "belly band" coils are off [Fig. 2-12(a)], but the ratio Rnhnc has been decreased by more than a factor of 2 compared to Figs. 2-10(b), 2-11(b), and 2-12(b). 
case 1 is $0.7 / 0.3=2.3$, which shows very close agreement. Hence, changes in the magnetic geometry can reduce the hot electron requirements (note that case 3 must also be investigated to make sure other requirements such as MHD stability are satisfied; this case was shown only to demonstrate the dependence on $R_{m}$ ). It should also be noted that this is only a point axial comparison. Volume integrals over the magnetic field profile would be required to determine the actual change in the ECRH power. Further work is being done to investigate the weaker dependence on $\left(T_{e c} / T_{j}\right)^{1 / 2}$ and $\left(\varphi_{\mathrm{e}} / \mathrm{T}_{\mathrm{ec}}\right)^{1 / 2}$

Yet another way to reduce the ECRH power is to decrease the axial extent of the hot electron density; this is controlled by the parameter Bh. In the above three cases, the hot electrons extended to the peak of the inner mirror $(B h \sim 2.2 \mathrm{~T})$. In Fig. 2-14(a) and $F i g .2-14(\mathrm{~b})$, Bh was varied in the normal TMX-(N) magnet configuration from $2.0 \mathrm{~T}$ to $1.4 \mathrm{~T}$. (Refer to Fig. 2-10(a) to see that $B=1.4 \mathrm{~T}$ approximately halfway up the inner magnetic field peak). The value of the peak potential is about the same in each case, and the profile is only a little flatter near the inner mirror peak. As Bh was decreased below 1.4 T, however, the hot electron density was not sufficient to maintain a smooth profile. These preliminary results indicate, at least from the standpoint of potential, that the hot electron density should extend about halfway up the mirror peak.

\subsection{SUMMARY}

In conclusion, the computer code for the potential model has shown that $n_{i t}$ and $n_{e h}$ are very important parameters and must have the proper values to achieve potentials of $I \varphi_{e} / T_{e c} \mid>5$. The trapped ion density is a result of pumping and will be discussed in Section 3 . The power required to sustain the hot electrons can bu minimized by increasing the mirror ratio $\left(R_{m}\right)$ and decreasing the axial extent of the density $(B h)$ without substantially changing the potential profile. Only modest changes are possible in $T M X(N)$ but future magnet designs can capitalize on these characteristics. Extensions of this model are continuing and will include the influence of the background neutrals on the potential profile and a study of the sensitivity to the parameter "s" (hot-electron distribution function) parameter. Finally, the details of the profile from the outer mirror peak to the wall will be worked out. 

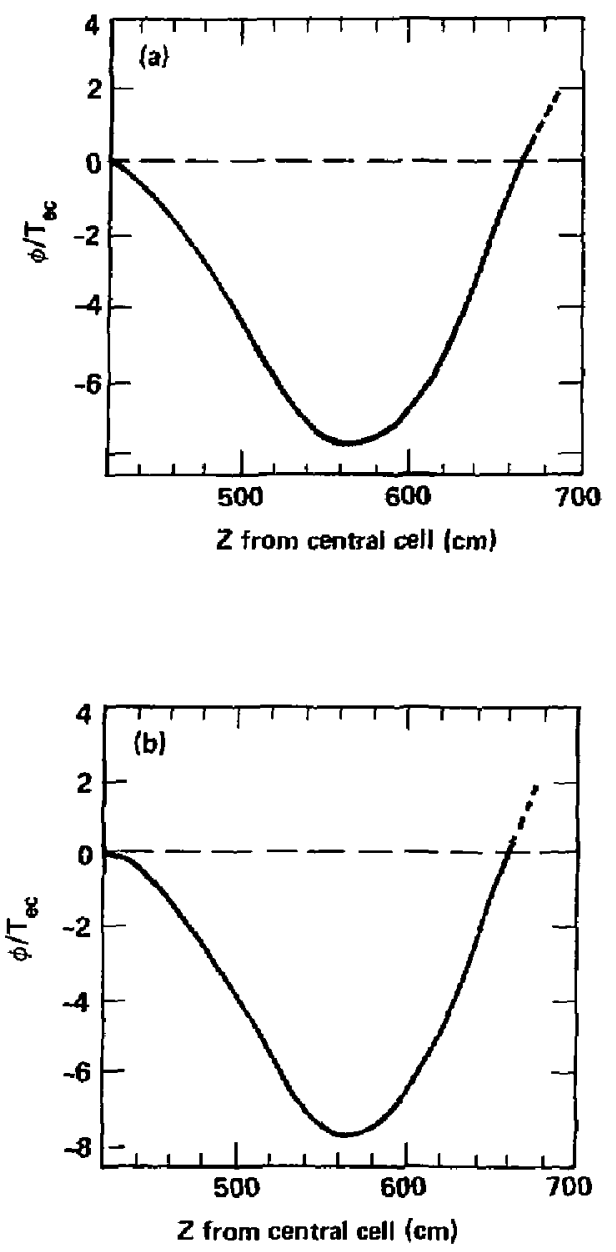

Figure 2-14. A comparison of the axial potential profile for the cases: (a) the hot-electron densjty extends to $B=2.0 \mathrm{~T}$ and $(\mathrm{b})$ the hot-electron density extend to $\theta=1.4 \mathrm{~T}$. 
SECTION 3

PUMPING OF NEGATIVE POTENTIAL WELL

(P. Poulsen)

The large negative potentials needed for confinement of central-cell electrons are created by removing trapped ions from the potential well. The method of pumping ions from the low potential regio: is charge exchange of the trapped ions with neutral beams aimed along the field lines. The calculation of the trapping rate for a square magnetic well has been performed by LoDestro. ' The potential is computed self-consistently. Using a Fokker-planck code, Futch has recently investigated the effect of more realistic magnetic field geometries, such as parabolic shapes and the TMX-Upgrade field configuration. These shapes tend to increase the irapping rate as compared to the rate for the square magnetic we11. For example, Futch has found an increase in the trapping rate by a factor of 22 for the TKX-Upgrade magnetic geometry. Logan, Futch, and Porter have each produced analytic fits to the results of the Fokker-Planck calculation. Logan's fit, good for square weils with the trapped ion density $n_{j t}$ about equal to the passing ion density $n_{\text {ip, is, }}$

$$
J_{\text {trap }} \frac{n_{i p}^{2}}{2.3 \times 10^{9} T_{i}^{3 / 2}}\left(1+0.2 R_{n}\right) \text {, }
$$

where $T_{j}$ is the central-cell ion temperature (in KeV). The fits of Porter and Futch are applicable for $n_{i t}>n_{i p}$, i.e. values of $g \equiv\left(n_{i t}+n_{i p}\right) / n_{i p}$ being greater than two. As an example, Porter's fit for mirror ratios of 2, 5,10 and 20 are shown in Fig. 3-1, transcribed from his original memo. 2 The yariable that expresses the pumping rate relative to the filling rate is $v_{p} T_{i}^{3 / 2} / n_{i p}$, where $v_{p} \equiv n_{\text {beam }}<\sigma v>$ for $n_{i p}\left(\mathrm{~cm}^{-3}\right)$ the density of passing ions at the bottom of the potential well.

The curves of $v_{p} T_{i}^{3 / 2} / n_{i p}$ vs $g$ can be convented to a relation between the filling parameter $g$ and the required neutral-beam current density for a specific magnetic geometry and plasma parameters. The pumping requirements for a square magnetic well are calculated first. The factors that account for effect of the actual magnetic geometry, the access of the pump beams to the 


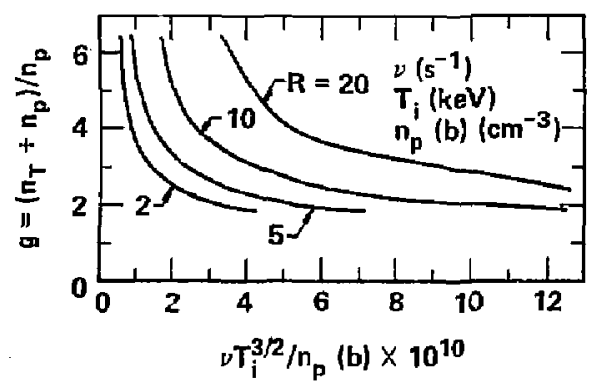

Figure 3-1. Density ratio $g$ vs neutral beam pumping parameter $u T_{i}^{3 / 2} / n_{p}(b)$. 
trapped ions in the plasma, and the mirror trapping of ions from the pump beam neutrals are then computed. The final result should then be a resanable estimate of the actual beam current that is needed to remove ions from the law-potential region.

The required neutral-beam pumping for a given value of $\mathrm{g}$ is computed as follow: From Fig. 3-1, the value of $v_{p} F_{i}^{3 / 2} / n_{i p}$ is determined. The pumping frequency $v_{p}$ is then computed if the values of the central-cell ion temperature $T_{i}$ and the total plug ion density $n_{T O T}=g n_{i p}$ is known. The neutral-beam current density $\mathrm{J}$ is then, for a square magnetic well,

$J=e v / o_{C X}$.

The effect of a real magnetic geometry is to increase the required pumping by a factor of 2 .

The neutral beams do not pump the p?asma uniformly cuer the whole low-potential region. Due to beam access requirements, the beams enter the plasma at an angle of $18^{\circ}$ to the magnetic axis. As a consequence, the beam current density varies throughout the pumping region. Furthermore, the field lines curve and produce the result that a fraction of the ions created by the pumping process are mirror confined in the low-potential region. These effects have been discussed by simonen ${ }^{3}$ for the TMX(P) configuration. The $f$ inite size of the pump beams and the effect of the geometry of the beam injection result in an effective path length of $70 \mathrm{~cm}$ through the plug plasma for each of four pump beams. The nonuniform pumping over the plug volume is not madeled by the Fokker-Planck code. This code assumes an axially symmetric potential profile in the end cell extending from mirror to mirror. A gross correction is effected by using:

$$
v_{p}=\left(\frac{70}{300}\right) J_{\text {ave }}{ }^{\sigma} c x
$$

where (70/300) is the ratio of the the effective pumping length to the mirror-to-mirror distance. The average neutral beam current derisity required to pump a potential well is then calculated for a given value of $\mathrm{g}$ by obtaining $v_{p} T_{i}^{3 / 2} / n_{i p}$ from $F i g .3-1$ and correcting for the magnetic field and the beam geometry. Thus, 


$$
J_{\text {ave }}=\left(\frac{300}{70}\right) * 2 *\left(\frac{v_{p} T^{3 / 2}}{n_{i p}}\right) \frac{{ }^{n} T 0 T}{g T_{i}^{3 / 2} \sigma_{c x}}
$$

The average current density is plotted in Fig. 3-2 for a mirror ratio of five. The number of neutral-beam modules corresponding to the indicated current density may be estimated by using an average current density of $0.1 \mathrm{amp} / \mathrm{cm}^{2}$ per bearm.

For successful pumping, the ion created as a result of charge exchange must be in the loss-region of the ion velocity space. The equation for the intersection of the ion loss boundary with the beam injection geometry is,

$$
\sin ^{2} \theta(z)=\frac{\sqrt{T-\beta(z)}}{R(z)}\left\{1-\frac{e\left[\phi(z)-\varphi_{e c}\right]}{E}\right\},
$$

$$
\text { where } \begin{aligned}
z & =\text { local axial coordinate } \\
\Theta & =\text { angle of injection } \\
R & =B_{M} / B(z) ; B_{m}=\text { inner mirror field } \\
\Phi_{C C} & =\text { central-cell potential } \\
\Phi(z) & =10 c a l \text { potential } \\
E & =\text { ion energy injected } \\
B(z) & =\text { axial beta profile. }
\end{aligned}
$$

For a $21^{\circ}$ injection angle, (average angle plus beam divergence) the mirror field greatly enhances the energy of ions that can be trapped by the low potential. Fig. 3-1 shows the mirror ratio that will result in trapping for an ion created at the bottom of the magnetic well. As an example, at the potential minimum $\varphi_{e}, B=0.30, R=4$, and $e \varphi_{e^{\prime}} / E=-0.39$. Herice, if e. ${ }_{\mathrm{e}} / \top_{\mathrm{ec}}=-7$ with $\mathrm{T}_{\mathrm{ec}}=500 \mathrm{eV}$, the beam energy that will result in trapping is $9.1 \mathrm{keV}$. Thus, boith the low energy components of a $17 \mathrm{keV}$ beam can te trapped in the potential region. Hence a substantial fraction of the charge exchange events result in no net pumping, but rather cause a redistribution of the trapped ions in both physical space and velocity space. The decrease in pumping effectiveness and the consequence of the alteration of the energy dependence of the trapped ions on the shape and depth of the potential is still to be investigated in detail. 


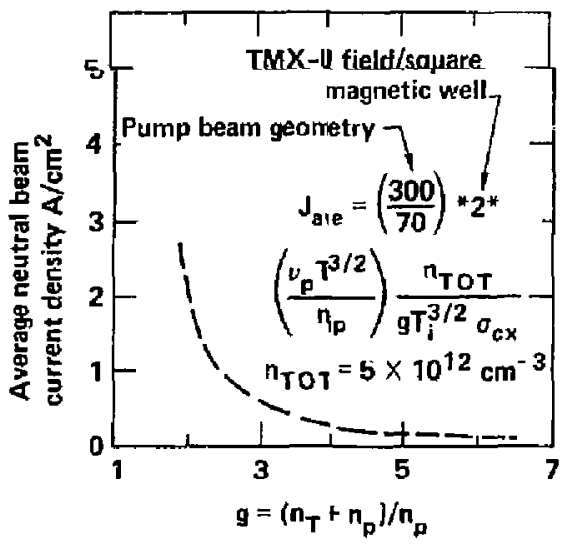

Figure 3-2. Average neutral-beam current density vs density ratio g. 
It is advantageous for negative michines to have a large plug mirror ratio. From Fig. 3-3 it is noted that this requirement does not appear to be compatible with the non-trapping requirements of beam pumping. However, both requirements can be satisfied if the plug mirror ratio to the central cell is large and the mirror field to the outside of the plug is sufficiently sma11:0 allow escape of the pump ions. For the preceding example above, an outside mirror ratio of 3.0 is sufficiently low to allow escape of the pump ions from a nominal 17-keV beam after a single bounce from the inside mirror. 


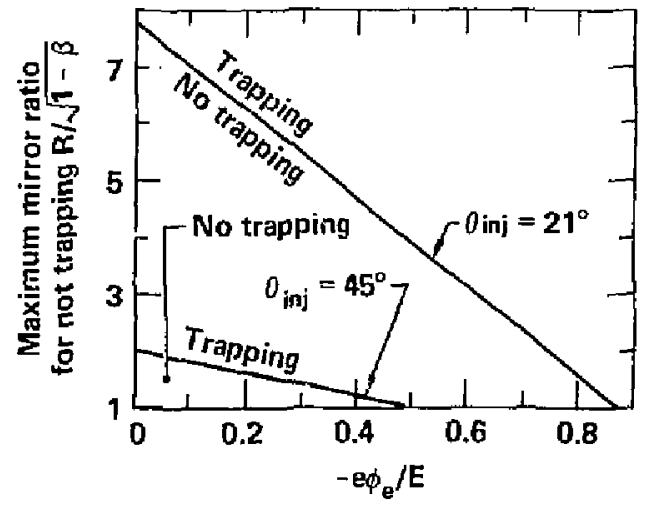

Figure 3-3. Maximum mirror ratio for non-trapping of charge exchanged pump beam neutrals. 
$-44-$ 
SECTION 4

FEEN OF HOT ELECTRON POPULATION

(W. E. Nexsen, B. H. Stallard)

A continuous feed of electrons to the mirror-trapped hot-electron population in the plugs is necessary to balance losses by scattering and $\mathrm{rf}$ diffusion. In principle such a feed can be provided in several different ways:

- Electron beam injection along field lines from ends

- Central-cell thermal electrons.

A feed system using electron beam injection from the ends would require a nonadiabatic mechanism such as a beam-plasma instability for trapping. It could replace expensive ECRH power with relatively cheap accelerator power and alter restrictions on plug densities which are presently set by available gyrotron frequencies. This is a high risk approach with potentially high gain which should be pursued. For the purposes of this report, however, we will consider an alternate approach of feeding from the central-cell thermal electrons.

In the negative potential mode of ipeiation, the central-cell thermal electrons are cut off at the potential minimum. This condition is to be contrasted with the positive mode where thermal electrons reach the outer mirrors and the feed can be located anywhere in the plug. When the negative mode of operation was first suggested at the $t$ ime of the development of the idea of a thermal barrier, a major objection was raised that the maximum hot-electron density would occur wherever the resonant feed was located, resulting in the potential minimum also accurring at this point and choking off the feed. Fokker-Planck runs have since shown that due to pitch angle scattering this will not occur if the feed is not located at the magnetic field minimum. These runs yield a distribution whose density is peaked at the field minimum and drops monotonically to zero at the field value of the peak of the lowest mirror. Thus, even though the hot electron density and, consequent $1 y$, the potential minimum peak at the field minimum, we still can locate the electron feed on the central-cell side of the field minimum. Since the potential at the feed point is $\Delta \phi$ greater than the value at the minimum, some density of thermal electrons will be present which may be used to provide the electron feed. Figure 4-1 sketches the magnetic field and the required potential and density variation. 


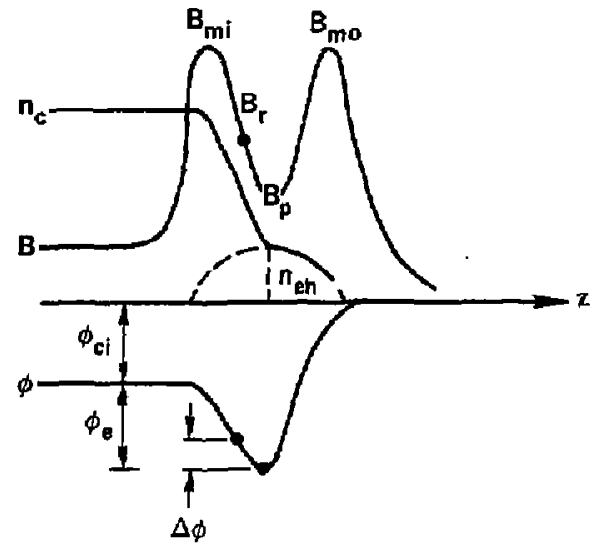

Figure 4-1. Typical profiles of density, magnetic field, and electrostatic potential showing the resonance point $B_{r}$. 
That the presence of such thermal electrons does not necessarily guarantee the ability co feed the hot electron population is illustrated in Fig. 4-2 where we sketch the electron velocity space diagram at the feed point for two possible cases. In the sketches, the region labeled $P$ is occupied by electrons confined by the potential $\Delta \phi$ and passing to the central cell, $Y$ is the region of Yushmanov electrons trapped between the inner mirror and the potential $\Delta \phi, M$ is the region of hot, mirror-trapped electrons, and $L$ is the region of electron loss. The intercepts of these various regions with the $V_{1}$ and $V_{I I}$ axis are given by

$$
\begin{aligned}
& v_{a}=\left[\frac{e 2 \Delta \phi}{m\left(1-B_{p} / B_{r}\right)}\right]^{1 / 2}=\left(\frac{2 E_{a}}{m}\right)^{1 / 2}, \\
& v_{b}=\left[\frac{e 2\left(\phi_{e}+\phi_{i}-\Delta \phi\right)}{m\left(\frac{m o}{B_{r}}-1\right)}\right]^{1 / 2}=\left(\frac{2 E_{b}}{m}\right)^{1 / 2}, \\
& v_{c}=\left[\frac{e 2\left(\phi_{e}-\Delta \phi\right)}{m\left(B_{m i} / B_{r}-1\right)}\right]^{1 / 2}=\left(\frac{2 E_{c}}{m}\right)^{1 / 2},
\end{aligned}
$$

and

$$
v_{L}=\left(\frac{e 2 \Delta \phi}{m}\right)^{1 / 2},
$$

where $B_{r}$ is the magnetic field at the resonance point for Ecluh.

in the feed process thermal electrons passing from the central cell encounter an ECRH region at $B_{r}$ (see :ig. 4-1) and, as a result, there is a net flux of electrons diffusing towards higher $V_{1}$ across the boundary between passing and Yushmanov particles and on out of the top of the Yushmanov region. Figure 4-2(a) illustrates a possible condition where a gap exists between the top of the Yushmanov region and the bottom of the mirror-trapped region which occurs if 
(a) Possible condition

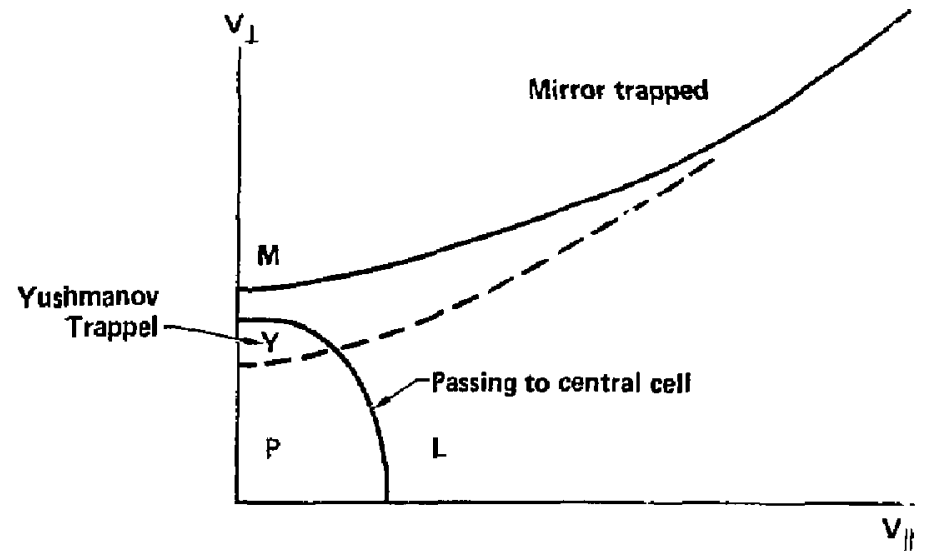

(b) Required condition of Yushmanov bridge

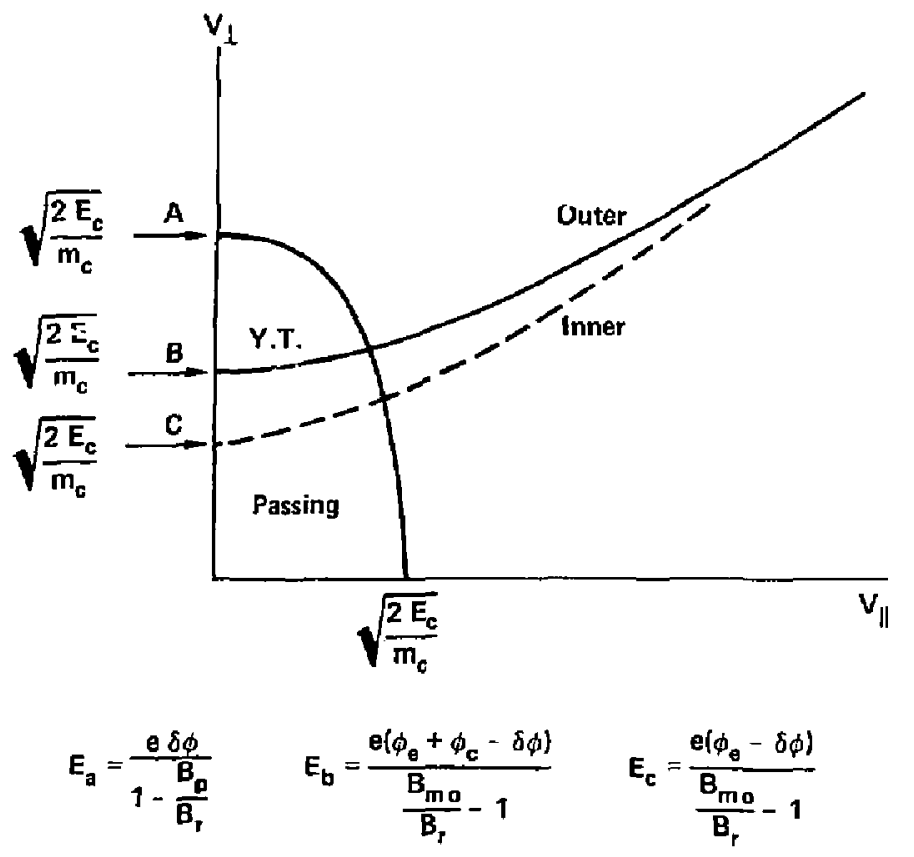

Require: $E_{z}>E_{b}$

Figure 4-2. Feed condition of inner $B_{r}$. (a) Possible condition. (b) Required condition of Yushmanov brjige. 


$$
v_{a}=\left[\frac{e 2 \Delta \phi}{m\left(T-B_{p} / B_{r}\right.}\right]^{1 / 2}<\left[\frac{e 2\left(\phi_{e}+\phi_{i}-\Delta \phi\right)}{m\left(\frac{m o}{B_{r}}-1\right)}\right]^{1 / 2}=v_{b} .
$$

For this condition feed from the thermal electrons is not possible since they will be last before they can be mirror trapped. Figure 4-2(b) the condition that is required to feed the hot electrons from the thermal electrons, that is,

$$
v_{a}>v_{b}
$$

so that an overlap, a Yushmanov "bridge", exists between the thermal electrons and the hot electrons.

For the particular case of TMX(N) with an existing magnetic field design, $B_{r}$ set to $10 \mathrm{kG}$ by the 28-GHz gyrotron frequency and $\phi_{e}$ and $\phi_{\mathbf{i}}$ determined for the base set of parameters, we wish to know whether we will satisfy the conditions for a Yushmanov bridge. To do this we have constructed a crude analytic model from which we estimate $\Delta \phi$ to determine the conditions for which a bridge will be obtained.

To minimize sheath formation, the potential minimum must be located at the minimum of the end-cell magnetic field. This requires that the ion-ciensity distribution decrease monotonically from the peak of the inner mirror to the minimum of the end-cell field. It is assumed that such a distribution can be produced by adjusting the magnitude and rocation of the trapped-ion pumping. We model the variation in ion density in this region by

$$
n_{i t}(B)=n_{c}\left(\frac{B}{B_{M i}}\right)^{k} \text {. }
$$

where, as discussed in Section $x, k \approx 0.6$.

To determine $\Delta \phi$ we invoke the quasi-neutrality condition at the feed position and at the field minimum 


$$
\begin{aligned}
& n_{j}\left(B_{r}\right)=n_{c}\left(\frac{B_{r}}{B_{M i}}\right)^{k}=n_{e c}\left(B_{r}\right)+n_{e y}\left(B_{r}\right)+n_{e h}\left(B_{r}\right) \\
& n_{i}\left(B_{p}\right)=n_{E}\left(\frac{B_{P}}{B_{M T}}\right)^{k}=n_{e h}\left(B_{p}\right)
\end{aligned}
$$

He:e $n_{e c}$ is the density of thermal electrons, $n_{e y}$ is the density of Yushmanoy trapped electrons and $n_{e h}$ is the density of the mirror-trapped ho: electrons. He introduce the parameter

$$
\alpha=n_{e h}\left(B_{r}\right) / n_{e h}\left(B_{p}\right)
$$

whose value may be obtained from Fokker-Planck runs or may be est imated fron

$$
\alpha \approx \frac{B_{m i}-B_{r}}{B_{m i}-B_{p}} \text {. }
$$

Using $c$ o eliminate $n_{\mathrm{eh}}$ from (7) and (8) we obtain

$$
\left.\frac{n_{c}}{R_{i}^{k}}[]-\alpha\left(\frac{B_{p}}{B_{r}^{k}}\right)^{k}\right]=n_{e c}\left(B_{r}\right)+n_{e y}\left(B_{r}\right),
$$

where $R_{i}=B_{m i} / B_{r}$

We mode? the variation of $n_{e c}$ and $n_{e y} w:$ th $\Delta \phi$ and solve (9) to obtain $\Delta \phi$. Figure 4-3(a) sketches the variation of the distribution function with $V_{1}$ for $V_{11}=0$. The parameter $E$ represents the effect of the ECRH electric field strengt's and $v_{m}$ is determined by the maximum energy $\left(E_{m}=i / 2 m v_{m}^{2}\right)$ above which the relativistic increase in mass results in the electron no longer being in resonance with the applied frequency within the feed zone as discussed later in this section.

In $F i g .4-3(b)$ we plot $f\left(V_{1}\right)$, the velacity distribution integraied over $v_{11}$, as a function of $v_{1}$. The increase of $f_{1}\left(v_{1}\right)$ with $v_{1}$ for the mirror-trapped group reflects the increase of available phase space volume filled by angle scattering. The densities of the various electron components is given by 


\section{Model density distributions}

(a)

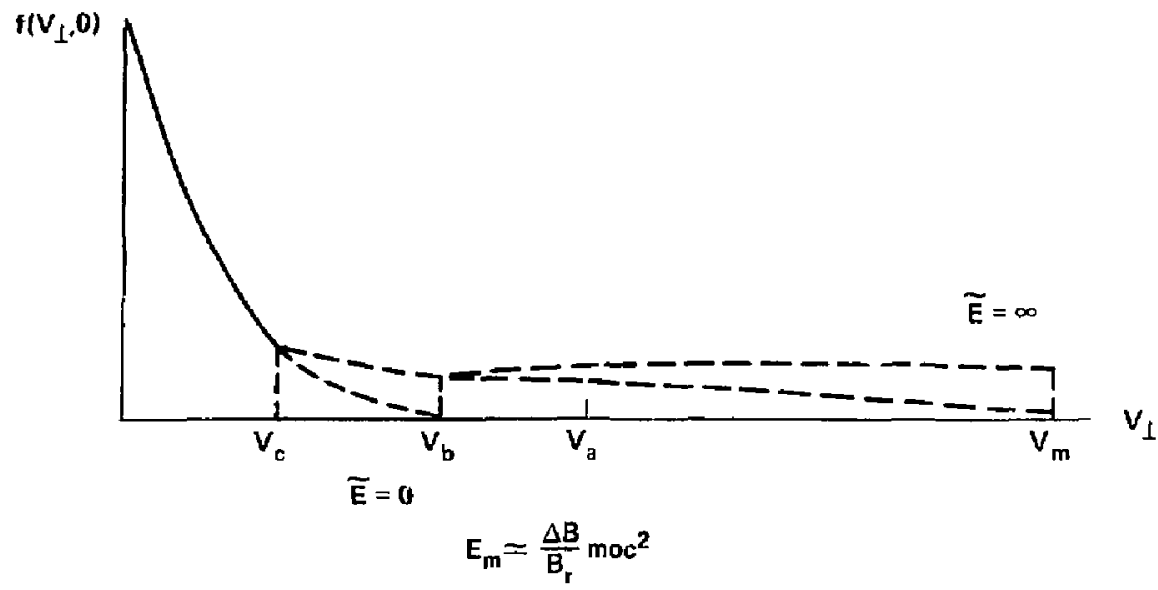

(b)

$f_{\perp}\left(V_{\perp}\right)=\int f\left(v_{\perp}, V_{\|}\right) \delta v_{\|}$

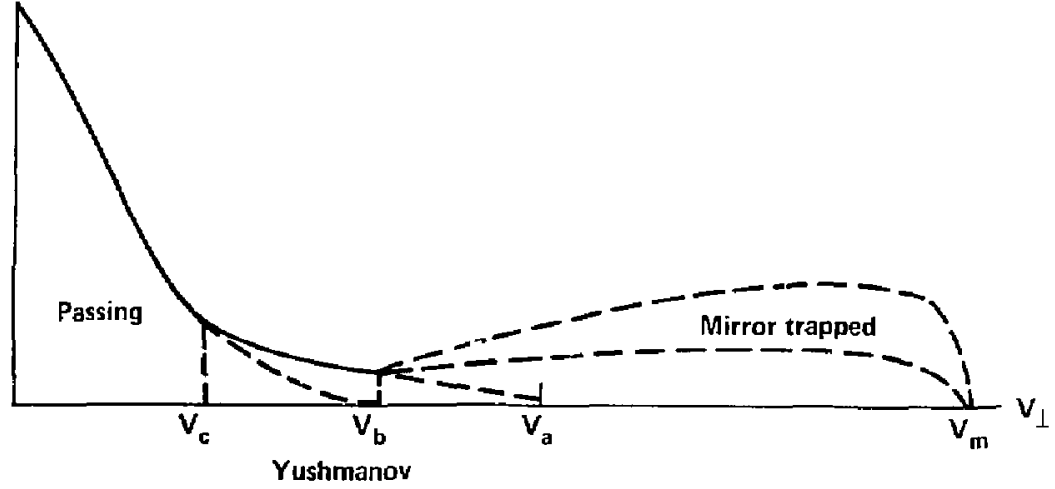

$$
\begin{aligned}
& n_{e c}=2 \pi \int_{0}^{v_{c} f_{\perp}}\left(v_{\perp}\right) v_{\perp} \delta v_{\perp} \\
& n_{e v}=2 \pi \int_{v_{c}}^{v_{2}} f_{\perp}\left(v_{\perp}\right) v_{\perp} \delta v_{\perp} \\
& n_{e h}=2 \pi \int_{v_{b}}^{v_{m}} f_{\perp}\left(v_{\perp}\right) v_{\perp} \delta v_{\perp}
\end{aligned}
$$

Figure 4-3. Model density distributions. 


$$
\begin{aligned}
& n_{e c} \approx 2 \pi \int_{0}^{v_{c}} f_{1}\left(v_{1}\right) v_{1} d v_{1}, \\
& n_{e y} \approx 2 \pi \int_{v_{c}}^{v_{a}} f_{1}\left(v_{1}\right) v_{1} d v_{1},
\end{aligned}
$$

and

$$
n_{e h} \approx 2 \pi \int_{v_{a}}^{v_{m}} f_{1}\left(v_{1}\right) v_{1} d v_{1} .
$$

For the passing (cold) electrons we use a distribution which is cut off at $\Delta \phi=0$ but approaches a Maxwellian for $\Delta \phi$ large

$$
f_{1}\left(v_{1}\right)=\frac{{ }^{\prime \prime} c}{T_{e c}}\left(\frac{m}{2 \pi}\right)\left\{\frac{\exp \left[-e\left(\varphi_{e}-\Delta \varphi\right) / k T_{e c}\right]-\exp \left[-e \varphi_{e} / k T e c\right]}{T-\exp \left(-e \phi_{e} / k T_{e c}\right)}\right\} \exp \left(-m v_{\perp}^{2} / 2 k T_{e c}\right) .
$$

Setting $x=\frac{e\left(\phi_{e}-\Delta \phi\right)}{k T_{e c}}$ and $y=e \phi_{e} / k T_{e c}$ we obtain

$$
n_{e c} \simeq n_{c} \frac{e^{-x}-e^{-y}}{1-e^{-y}}\left[1-\exp \left(-E_{c} / k T_{e c}\right)\right],
$$

where

$$
\frac{E_{C}}{K T_{E Z}}=\frac{e\left(\phi_{e}-\Delta \phi\right)}{K T_{e c}\left(R_{i}-T\right)}=\frac{x}{R_{i}-T} .
$$

For the ECR-heated case we approximate the distribution of the Yushmanov confined electrons at $\mathrm{B}_{r}$ by

$$
\left.f_{1}\left(v_{1}\right)=\frac{n_{c}}{k T e c}\left(\frac{m}{2 \pi}\right)\left(\frac{e^{-x}-e^{-y}}{1-e^{-y}}\right) \exp :-E_{c} / k T_{e c}\right)\left[1-\rho_{1} \frac{\left(v_{1} / V_{c}-1\right)}{\left(V_{a} N_{c}-1\right)}\right]
$$


for $v_{1}$ in the range $v_{c} \leq v_{1} \leq v_{a}$. Here, $\rho_{1}$ is a parameter which is a function of the ECRH field and approaches zero as $E_{c}$ becomes very large. Solving Eq. (11) for this distribution gives for the density of the Yushmanov electrons

$$
\begin{aligned}
n_{e y}= & n_{c}\left(\frac{e^{-x}-e^{-y}}{1-e^{-y}}\right) \exp \left(-E_{c} / k T_{e c}\right)\left[\left(\frac{E_{a}}{k T_{e c}}-\frac{E_{c}}{k T_{e c}}\right)\left(1+c_{1}\right)\right. \\
& \left.-\frac{2}{3}\left(\frac{\left(E_{a} / k T_{e c}\right)^{3 / 2}-\left(E_{c} / k T_{e c}\right)^{3 / 2}}{\left(E_{c} / k T_{e c}\right)^{1 / 2}}\right) c_{1}\right],
\end{aligned}
$$

where

$$
\frac{E_{a}}{K T_{e}}=\frac{e A \phi}{R T_{e} e^{\left(1-B_{p} / B_{r}\right)}}=\frac{y-x}{\left(1-B_{p} / B_{r}\right)}
$$

and

$$
c_{1}=\frac{\rho_{1}}{\left(E_{a} / E_{c}\right)^{1 / 2}-1} .
$$

Substituting (14) and (16) into (9) yields

$$
\begin{aligned}
& F\left(x, \rho_{i}\right) \equiv\left(B_{p} / B_{r}\right)^{k}-\frac{1}{\alpha} 1-R_{i}^{k}\left(\frac{e^{-x}-e^{-y}}{1-e^{y}}\right)\left[1-\exp \left(-E_{c} / k T_{e c}\right)\right] \\
& +e^{-E_{c} / k T_{e c}}\left[\left(\frac{E_{a}}{k T_{e c}}-\frac{E_{c}}{k T_{e c}}\right)\left(1+c_{1}\right)-2 / 3\left(\frac{\left[\left(E_{a} / k T_{e c}\right)^{3 / 2}-\left(E_{c} / k T_{e c}\right)^{3 / 2}\right]}{\left(E_{c} / k T_{e c}\right)^{1 / 2}}\right) c_{1}\right] \\
& =0
\end{aligned}
$$


For the Yushmanov bridge we require

$$
E_{a}>E_{b}=\frac{e\left(\phi_{e}+\phi_{i}-\Delta \phi\right)}{B_{m o} / B_{r}-T}
$$

Solution of (17) yields the conditions for which the Yushmanov bridge wili be obtained. Figure 4-4 gives the variation of $E_{a} / E_{b}$ with $B_{p} / B_{r}$ with $\rho_{1}$ as a parameter for Case A (see Section ig) operating parameters. For the base case magnetic field configuration with $B_{p} / B_{r}=0.5$ for the vacuum field which is beta depressed to $B_{p} / B_{r} \approx 0.4$, the existence of a bridge is marginal. The existence of a bridge appears much more certain if $B_{p} / B_{r}$ is increased. In this mode of operation we conclude that the fiug magnetic field should be increased from the base case value to a vacuum field ratio $B_{p} / B_{r}>0.6$.

We turn now to the modeling of the hot electron behavior. In addition to satisfying the quasineutrality condition, given by Eq. (8) for our model, there are certain constraints on the average energy of the mirror-trapped electrons imposed by MHD stability conditions and by ECRH power considerations. For the positive model of operation an average hot electron energy of $50 \mathrm{keV}$ was chosen as the best compromise. We adopt this value as a goal for negative operation to see how our parameters are affected. To obtain this electron temperature, we must avoid hot electron runaway to much higher energy. To accomplish this, electron resonance at high energy ( $E>E_{m}$ as shown in Fig. 4-3) is reduced by limiting the $r f$ inwer to magnetic field region

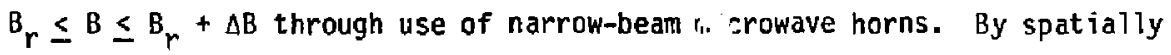
limiting the power, the resorance at high-energy shifts to regions of weak $r f$ fields, thereby limiting heating.

The resonance condition is given by

$$
\frac{n \omega_{c e}}{\gamma}=\omega_{r f} \pm k_{\| l} v_{\|}
$$

for harmonic number $n$ and $\gamma=\left[1-(v / c)^{2}\right]^{-1 / 2}$. For the simple case $k_{11}=0$, the maximum resonant energy is given by

$$
\frac{E_{\max }}{m_{e c}^{2}}=r_{\max }-1=\frac{\Delta B}{B_{r}} \text {, }
$$




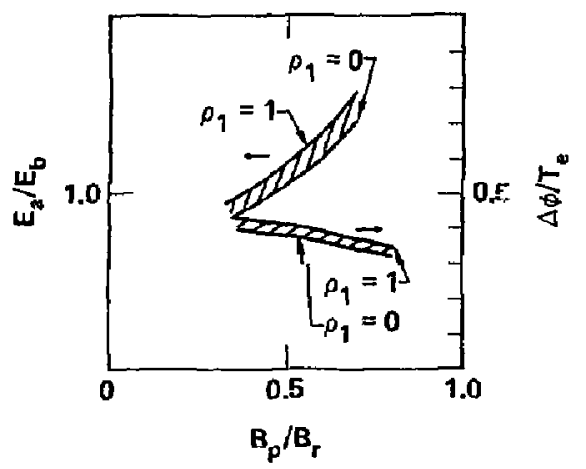

Figure 4-4. Condition for satisfying the Yushmanov bridge condition as a function of the magnetic fjeld magnitude at resonance. 
where $B_{r}$ is the magnetic field for "cold plasma" resonance at hamonic number $n$. The maximum energy is, in addition, subject to the restriction $\gamma_{\max }<n+1 / n$ which is the condition that the next higher harmonic $(n+1)$ resonance does noi overlap the $n^{\text {th }}$ harmonic due to the relativistic broadening of the absorption. For nonzerr, $k_{\|}$, Doppler effects broaden the resonance and the situation is mor: complicated. However, for realizable experimental conditions, it still ar.pears feasible to limit the heating for energies of interest for TMX-Upgrade. The distribution of mirror trapped electrons at $B=B_{r}$ is modeled by

$$
f_{\perp}\left(V_{\perp}\right) \approx f_{\perp}\left(E_{b}\right)\left[1+\left(\frac{V_{\perp}}{V_{b}}-1\right)\left(R_{0}-1\right)^{1 / 2}\right]\left[1-\frac{\rho_{2}\left(V_{\perp} / V_{b}-1\right)}{\left(V_{m} / V_{b}-\eta\right)}\right] \text {. }
$$

where $R_{0}=B_{m o} / B_{r}$ and $\rho_{2}$ a fres parameter. (We introduce a small error by counting the volume between $v_{\perp}$ and $v_{\mathrm{d}}$ twice). The first term in square brackets approximates the increase in phase-space volume with $v_{\perp}$ assuming the distribution spreads to the loss-cone toundary while the second term models the effect of ECRH on the distribution function. In introducing models for the Yushmanov distribution and the mirror distribution with their parameters $\rho_{1}$ and $\rho_{2}$, we have assumed that trapping and heating of the mirmo-trapped e lectrons can, to a degree, be decoupled by ECRH heating at more than one resonant zone, i.e., trapping in thr resonant zone on the central-cell side of the nlug minimum and heating in the resonant zone between the plug minimum and the outer mirror where no thermal electrons are present. Defining the carameters $c_{2}$ and $c_{3}$, Eq. (18) reduces to

$$
f\left(v_{1}\right)=f\left(E_{c}\right)\left(1-\rho_{1}\right)\left[\left(1-c_{3}\right)+c_{3} v_{1} / v_{b}\right]\left[\left(1+c_{2}\right)-c_{2} v_{1} / v_{b}\right]
$$

with

$$
C_{2}=\frac{\rho_{2}}{\left(E_{m} / E_{b}\right)^{1 / 2}-1}
$$

and

$$
c_{3}=\left(R_{0}-1\right)^{1 / 2}=\left(\frac{B_{0}}{B_{r}}-1\right)^{1 / 2} .
$$


Substituting (19) into (12) and integrating gives

$$
\begin{aligned}
n_{e h}\left(B_{r}\right) \approx & n_{c}\left(\frac{e^{-x}-e^{-y}}{1-e^{-y}}\right) \exp \left(-E_{c} / k T_{e c}\right)\left(1-\rho_{1}\right)\left(1 \cdot c_{3}+c_{2}-c_{3} c_{2}\right) \\
& \cdot\left(\frac{E_{m}}{k T_{e c}}-\frac{E_{b}}{k T_{e c}}\right)+\frac{2}{3}\left(c_{3}-c_{2}+c_{3} c_{2}\right) \frac{\left[\left(\frac{E_{m}}{k T_{e c}}\right)^{3 / 2}-\left(\frac{E_{b}}{k T_{e c}}\right)^{3 / 2}\right]}{\left(E_{b} / k T_{e c}\right)^{T / 2}} \\
& +\frac{1}{2} c_{3} c_{2} \frac{\left[\left(\frac{E_{m}}{k T_{e c}}\right)^{2}-\left(\frac{E_{b}}{k T_{e c}}\right)^{2}\right]}{\left(E_{b} / k T_{e c}\right)^{2}} .
\end{aligned}
$$

The quasi-neutrality condition at $B_{p}$ then requires that

$$
\begin{aligned}
G\left(\rho_{1}, \rho_{2}, \frac{E_{m}}{t_{e c}}\right) \equiv\left(B_{p} / B_{r}\right)^{k}-\left(R_{i}^{k} / \alpha\right)\left(\frac{e^{-x}-e^{-y}}{1-e^{-y}}\right) \exp \left(-E_{c} / k T_{e c}\right)\left(1-\rho_{1}\right) \\
\cdot\left\{\left(1-C_{3}+C_{2}-C_{3} C_{2}\right)\left(\frac{E_{m}}{k T_{e c}}-\frac{E_{b}}{k T_{e c}}\right)+\frac{2}{3}\left(C_{3}-C_{2}+C_{3} C_{2}\right)\right. \\
\cdot \frac{\left[\left(E_{m} / k T_{e c}\right)^{3 / 2}-\left(E_{b} / k T_{e c}\right)^{3 / 2}\right]}{\left(E_{b} / k T_{e c}\right)^{1 / 2}} \\
\left.-\frac{1}{2} C_{3} C_{2} \frac{\left[\left(E_{h} / k T_{e c}\right)^{2}-\left(E_{b} / k T_{e c}\right)^{2}\right]}{\left(E_{b} / k T_{e c}\right)^{2}}\right\}=0 .
\end{aligned}
$$

Since $k$ and $\alpha$ are more or less determined by pumping and scattering and $E_{m}$ is determined by placement of the ECRH feed (in the simplest model $E_{m} \approx\left(\Delta B / B_{r}\right) m_{0} C^{2}$ where the feed horn illuminates the zone $\left.B_{r}<B<B_{r}+\Delta B\right)$, the free parameters are mainly $\rho_{1}$ and $\rho_{2}$.

$-37-$ 
The average energy of the mirror trapped electrons, $\bar{E}$, is given by

$$
\bar{E} / T_{\text {ec }}=\frac{N}{D}
$$

where

$$
\begin{aligned}
N \equiv & 1 / 2\left(1-c_{3}+c_{2}-c_{3} C_{2}\right)\left[\left(E_{m} / k T_{e c}\right)^{2}-\left(E_{b} / k T_{e c}\right)^{2}\right] \\
& +\frac{2}{5}\left(c_{3}-c_{2}+2 c_{3} C_{2}\right) \frac{\left[\left(E_{m} / k T_{e c}\right)^{5 / 2}-\left(E_{b} / k T_{e c}\right)^{5 / 2}\right]}{\left(E_{b} / T_{e c}\right)^{1 / 2}} \\
& -\frac{c_{3} C_{2}}{3} \frac{\left.\left[E_{m} / k T_{e c}\right)^{3}-\left(E_{b} / k T_{e c}\right)^{3}\right]}{\left(E_{b} / k T_{e c}\right)}
\end{aligned}
$$

and

$$
\begin{aligned}
D \equiv & \left(1-c_{3}+c_{2}-C_{3} C_{2}\right)\left[\left(E_{m} / k T_{e c}\right)-\left(E_{b} / k T_{e c}\right)\right] \\
& +\frac{2}{3}\left(C_{3}-c_{2}+2 c_{3} C_{2}\right) \frac{\left[\left(E_{m} / k T_{e c}\right)^{3 / 2}-\left(E_{b} / k T_{e c}\right)^{3 / 2}\right]}{\left(E_{b} / k T_{e c}\right)^{1 / 2}} \\
& -\frac{c_{3} C_{2}}{2} \frac{\left.\left[E_{m} / k T_{e c}\right)^{2}-\left(E_{b} / k T_{e c}\right)^{2}\right]}{\left(E_{b} / k T_{e c}\right)} .
\end{aligned}
$$

The object of this modeling of the hot electrons is to estimate the values of $p_{1}$ and $P_{2}$ necessary to satisfy $E q$. (21) when $E_{m} / T_{\text {ec }}$ is such that $\bar{E}=50 \mathrm{keV}$ for the TMX-(N) conditions. The future goal is to relate these values of $\rho_{1}$ and $\rho_{2}$ to the $i n i$ : dent ECRH power, but for now we assume that enough ECRH power is available to give the required values of $\rho_{1}$ and $\rho_{2}$.

Fig. 4-5 gives the variation of $E_{m} / T_{e c}$ vs $\rho_{1}$ with $\rho_{2}$ as a parameter from the solution of (17) and (21) for the input parameters of case $A$ and a beta depressed value of $B_{p} / B_{r} \approx 0.5$. Fig. 4-6 gives the variation of $\bar{E} / E_{m}$ with $\rho_{2}$ from (22), (23), and (24). 


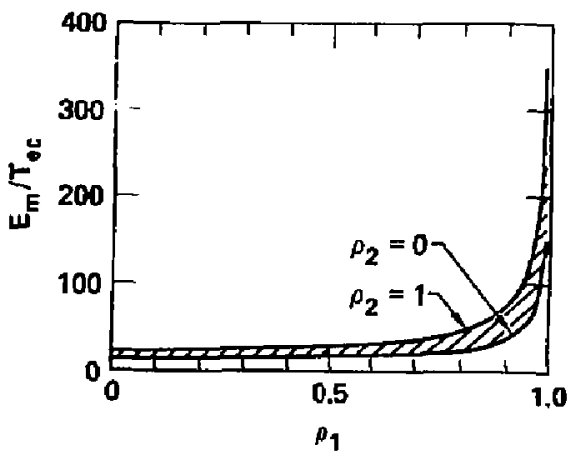

Figure 4-5. Maximum normalized energy $E_{m} / T_{e c} v 5$ the distribution function parameter $\rho_{j}$. 


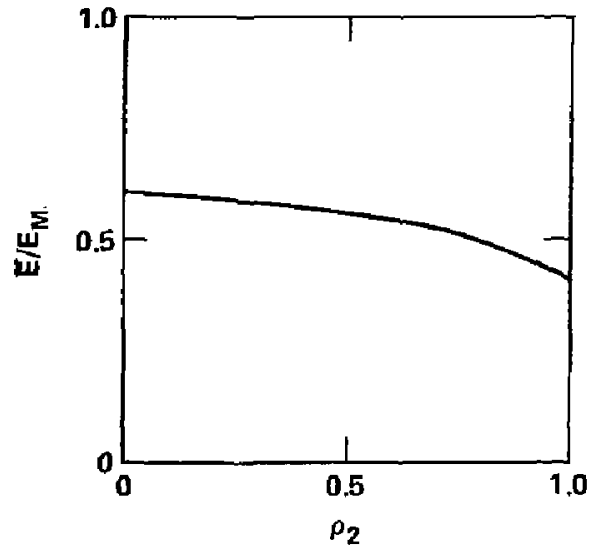

Figure 4-6. Average normalized energy $\bar{E} / E_{m}$ vs distribution function parameter $\rho_{2}$. 
From Fig. A-6 we see that $E / E_{m}$ lies in the range of $0.4-0.6$ depending on the value of $\rho_{2}$. Therafore, for $E=50 \mathrm{keV}$ we require that the feed be designed for $E_{m} \approx 100 \mathrm{keV}$ and since $T_{e c} \cong 0.5 \mathrm{keV}$ we require $E_{m} / T_{e c} \approx 200$. This means $\rho_{1}$ must have a value in the range of 0.98 to 0.99 or that $f_{1}\left(E_{a}\right) / f_{1}\left(E_{c}\right) \approx 0.01$ to 0.02 . While this value may seem smail, the value would be $0.001-0.004$ for a pureily Maxwellian tail, almost an order of magnitude lower. This enhancement of the tail by the ECRH is what is required to feed the hot electron plasma.

for a fixed $E_{m}$, it appears from Fig. $4-5$ that for $p_{1}$ in the range described above, large changes in $T_{e c}$ can be compensated for by small changes in $\rho_{1}$ and a large range of plasma parameters can be handled with the $\rho_{1}$ and $\rho_{2}$ knobs.

This model, while crude, gives hope that the proper conditions for feeding a negative plug and adjusting its energy will not be too difficult to obtain. Further refinement of the analytic model could be made, but quantitative answers to question of ECRH power requirements probably await the completion of codes that are now under development. 
$-62-$ 
SECTION 5

ECRH POWER REQUIREMENTS

(T. C. Simonen)

The purpose of this section is to compare the heating efficiencies of previous ECRH experiments as a bas is for comparison of TMK-Upgrade projections. Previous hot electron experiments have been carried out in minimum-B mirrors, EBT's, simple mirrors, and internal ring machines.

Microwave absorption efficiency can of ten be very high. However, it is not always clear how the power is ultimately absorbed. Individual experiments utilize ECRH power differentiy. Cur aim is to attempt to evaluate the efficiency with which microwave power is absorbed into the energetic electrons. Besides sustaining the hot electron density, ECRH power is required for ionization of gas, generation and heating of any colu plasma component, feer $\iota$ ing of tha hot component, sustaining nonadiabatic losses, overcoming electron microinstabilities, heating edge plasma, supplying waveguide and cavity losses. etc. The objective is to maximize the power absorbed in the hat electron group forming the negative potential end cell while minimizing the ECRH powe flow into these other channels. The expected perfor unance per; and of the ECRh system is:

TMX-Upgrade tube output ( 2 gyrotrons at $200 \mathrm{kl}:: \quad 400 \mathrm{~kW}$ Dutput of 8 arm couplers (20\% loss) $320 \mathrm{~kW}$ Output of waveguides (40\% loss) $190 \mathrm{~kW}$

Incident of resonant surface (20\% loss) $150 \mathrm{~kW}$

\subsection{PRESENT BEST ESTIMATES OF REQUIRED ECRH POWER}

Fokker-Planck runs by Stallard ${ }^{1}$ using Matsuda's code have indicated that the ECRH power required to maintain a hot-electron plasma against scattering into the loss cone is

$$
P=\frac{2.8 n_{e h}\left(0.46 T_{e h}\right)}{0.7 \tau_{e e} \log R}, W / c^{3},
$$


where $n_{e h}$ is the hot electron density, $T_{e h}$ is the hot electron temperature, $\tau_{e e}$ is the electron-electron $90^{\circ}$ scattering time, and $R$ is the mirr,$r$ ratio. The factor 2.8 accounts for angle scatter of botin electrons and ions. The code indicates the average energy loss is $0.46 \mathrm{~T}_{\mathrm{eh}}$. The factor 0.7 is due to the enhanced ECRH diffusion. Converting to practical units,

$$
P\left(\mathrm{H} / \mathrm{cm}^{3}\right)=5.7 \times 10^{-25} \pi_{\mathrm{eT}} \pi_{\mathrm{eh}}\left(\mathrm{cm}^{-3}\right) \mathrm{T}_{\mathrm{eh}}^{-1 / 2}(\mathrm{keV}) / \log \mathrm{R} .
$$

Here, $n_{e}$ is the total electron density (cold + hot, $\left.n_{e t}=n_{e c}+n_{e h}\right)$. Using ine above formula we calculate for $\operatorname{TMX}(\mathrm{N})$

$$
P=\frac{5.7 \times 10^{-25}\left(4 \times 10^{12} \mathrm{~cm}^{-3}\right)^{2}}{\left(\frac{2}{3} \cdot 50\right)^{1 / 2} \log 4} \frac{\left(0.33 \pi(20)^{2}\right.}{4} \underline{150)}=170 \mathrm{~kW},
$$

assuming a cylindrical plasma of $20-\mathrm{cm}$ radius and $150-\mathrm{cm}$ length with $\mathrm{n}_{\mathrm{ec}}=0$. The factor 0.33 is the $n^{2}$ volume weighting factor assuming a parabolic density profile. These calculations are somewhat optimistic since they do not include the effects of plasma potential, power absorbed by potential-confined electrons, or scattering off the passing and penetrating central-cell ions and electrons. This estimate is just under the anticipated 190-kW incident on the plasma. We expect some reduction in the power incident on the resonant surface depending on the antenna aiming, ray bending, and edge absorption. Small reductions in plasma radius or density cou id accommodate any difference, however, by comparing with previous experimental performance, more significant discrepancies could emerge.

But first, let's review how Stallard's present Fokker-Planck power estimates differ from those in the TMX(P) proposa]. ${ }^{2}$ The 170-kW power level differs from the $50 \mathrm{~kW}$ calculated in the proposal ${ }^{2}$ [Eq. (35), page A-40] for the reasons summarized in Table 5-1. 
Table 5-1. Increases in calculated microwave power due to improved theoretical madei ing.

\begin{tabular}{|c|c|c|c|c|}
\hline Parameter & $\begin{array}{l}\text { Present } \\
\text { estimate }\end{array}$ & $\begin{array}{l}\text { Proposal } \\
\text { estimate }\end{array}$ & $\begin{array}{c}\text { Reason for } \\
\text { change }\end{array}$ & $\begin{array}{c}\text { Change in } \\
\text { power } \\
\text { requirement }\end{array}$ \\
\hline$n_{e h}, c^{-3}$ & $4 \times 1012$ & $5 \times 1012$ & $\begin{array}{l}\text { Ray tracing } \\
\text { code (microwave } \\
\text { penetration) }\end{array}$ & 0.64 \\
\hline $\begin{array}{l}\text { Hot } \\
\text { electron } \\
\text { length, cm }\end{array}$ & 150 & 60 & $\begin{array}{l}\text { Fokker-planck } \\
\text { code }\end{array}$ & 2.5 \\
\hline$E_{\text {Loss }}$ & $0.3 E_{h}$ & $0.5 E_{h}$ & $\begin{array}{l}\text { Fokker-Planck } \\
\text { cade }\end{array}$ & 0.0 \\
\hline RF diffusion & $1.5 / \tau_{\mathrm{ee}}$ & $1 / \tau_{e e}$ & Fokker Planck & 3.4 \\
\hline \multirow[t]{2}{*}{$\begin{array}{l}\text { Electron- } \\
\text { ion } \\
\text { scattering }\end{array}$} & Included & Neglected & $-\infty$ & 2.8 \\
\hline & & Tatal chang & n power requirement & $=3.76$ \\
\hline
\end{tabular}

\subsection{COMPARISON OF THEORY WITH PREVIOUS ECRH EXPERIMENTS}

Table 5-2 shows a comparison of parameters and the applied power of several earlier experiments with the power requ, rements predicted by Ea. (2) for (a) CW experiments and (b) pulsed experiments. In addition, for each experiment the theoretic $\exists$ \} not electron lifetime is given;

$$
\tau_{\mathrm{e}}=0.7 \tau_{\mathrm{ee}} / 2.8=1.7 \times 10^{8} \mathrm{~T}_{\mathrm{e}}^{3 / 2}(\mathrm{keV}) \mathrm{n}_{\mathrm{er}}^{-1}\left(\mathrm{~cm}^{-3}\right) / \log \mathrm{R} .
$$

In making this comparison some uncertzinty exists regarding experimental parameters. Nevertheless several general trends emerge. The highest efficiency for microwave absorption is $10 x$ in FBT. This is the quoted fraction that goes into the hot electron ring. Eighty percent of the remaining power is accounted for as absorption into the toroidal and edge 
Table 5-2. Comparison of ECRH experiments.

a. CH Experiments

\begin{tabular}{|c|c|c|c|c|c|c|c|c|c|c|c|}
\hline Expertment & Ref. & $\begin{array}{c}n_{\text {eh }} \\
\left(\mathrm{cm}^{-3}\right)\end{array}$ & $\begin{array}{c}\mathrm{n}_{\mathrm{e}} \\
\left(\mathrm{cm}^{-3}\right)\end{array}$ & $\begin{array}{c}\mathrm{T}_{\text {eh }} \\
(\mathrm{keV})\end{array}$ & $\begin{array}{c}v \\
\text { (1iters) }\end{array}$ & $\mathrm{R}$ & $\begin{array}{c}P_{\text {applied }} \\
(\mathrm{kH})\end{array}$ & $\begin{array}{c}P_{\text {theory }} \\
(\mathrm{kW})\end{array}$ & $\frac{P_{\text {applied }}}{P_{\text {theory }}}$ & $\begin{array}{l}e \\
(5) \\
\end{array}$ & $\begin{array}{c}\text { Limiting hot } \\
\text { electron } 1055 \\
\text { process }\end{array}$ \\
\hline $\begin{array}{l}\text { Tandem mirror } \\
\text { TMX-Upgrade }\end{array}$ & 1,2 & $4 \times 10^{12}$ & a & 33 & 20 & 4 & 190 & 170 & 1.1 & 0.008 & Coutanb \\
\hline $\begin{array}{l}\text { Nintmum-B mirrors } \\
\text { CIRCE } \\
\text { Interm } \\
\text { Elmo }\end{array}$ & $\begin{array}{l}3 \\
4 \\
5\end{array}$ & $\begin{array}{r}3.5 \times 10^{11} \\
4 \times 10^{11} \\
1 \times 10^{12}\end{array}$ & $\begin{array}{c}2.2 \times 10^{11} \\
a \\
a\end{array}$ & $\begin{array}{r}100 \\
320 \\
1000\end{array}$ & $\begin{array}{l}? .5 \\
1.5 \\
1.0\end{array}$ & $?^{2}, 6$ & $\begin{array}{l}-- \\
0.9 \\
1.88\end{array}$ & $\begin{array}{l}-- \\
0.04 \\
0.06\end{array}$ & $\begin{array}{l}\overline{21} \\
30\end{array}$ & $\begin{array}{l}0.48 \\
2.43 \\
5.4\end{array}$ & $\begin{array}{l}\text { ? } \\
\text { Nonadiabatic } \\
\text { Nonadiabatic }\end{array}$ \\
\hline $\begin{array}{l}\text { Toroidal mirrors } \\
\text { EBT }\end{array}$ & 6 & - & $2-$ & -- & -- & -- & -- & -- & 10 & -- & Monadiabatic \\
\hline $\begin{array}{l}\text { Simple mirrors } \\
\text { STH } \\
\text { PTF } \\
\text { Interm } \\
\text { TPH }\end{array}$ & $\begin{array}{l}7 \\
5 \\
4 \\
5\end{array}$ & $\begin{array}{l}1 \times 10^{11} \\
3 \times 10^{11} \\
5 \times 10^{11} \\
? \times 10^{10}\end{array}$ & $\begin{array}{l}1 \times 10^{i 1} \\
a \\
a \\
1 \times 10^{12}\end{array}$ & $\begin{array}{l}370 \\
100 \\
200 \\
100\end{array}$ & $\begin{array}{c}? .5 \\
3 \\
50 ? \\
1\end{array}$ & $\begin{array}{l}2 \\
2 \\
2 \\
2\end{array}$ & $\begin{array}{l}1.0 \\
1.0 \\
50 \\
5\end{array}$ & $\begin{array}{l}0.005 \\
0.024 \\
1.77 \\
-.\end{array}$ & $\begin{array}{r}200 \\
208 \\
28 \\
--\end{array}$ & $\begin{array}{l}5 \\
0.57 \\
1.0 \\
1.6\end{array}$ & $\begin{array}{l}\quad b \\
\text { Nonadiabatic } \\
\text { Nonadiabat ic } \\
\text { Microinstabil ity }\end{array}$ \\
\hline $\begin{array}{l}\text { Internal rings } \\
\text { SCL }\end{array}$ & 8 & $1 \times 10^{10}$ & $2 \times 10^{11}$ & 250 & 50 & 10 & 1 & $0.000^{\circ}$ & 175 & 5.7 & 1 \\
\hline
\end{tabular}

asssumed equal to the hot-eTectron density.

$b \star \star$ Loss processes identified as $800 \mathrm{~W}$ to cold eiectrons, fon $\mathrm{W}$ to ionization by electrons and $100 \mathrm{~W}$ hot-electron nonadiabatic loss. 
Table 5-2. (continued)

b. Pulsed Experiments

\begin{tabular}{|c|c|c|c|c|c|c|c|c|c|c|c|c|}
\hline Experiment & $\begin{array}{c}n_{\mathrm{eh}} \\
\left(\mathrm{cm}^{-3}\right) \\
\end{array}$ & $\begin{array}{c}n_{\mathrm{e}} \\
\left(\mathrm{cm}^{-3}\right) \\
\end{array}$ & $\begin{array}{c}\mathrm{T}_{\text {eh }} \\
(\mathrm{keV})\end{array}$ & $\begin{array}{c}V \\
\text { (1iters) }\end{array}$ & $\mathrm{R}$ & $\begin{array}{c}P \\
(\mathrm{~kW})\end{array}$ & $\begin{array}{l}\Delta t \\
15:\end{array}$ & $\begin{array}{l}P \Delta t \\
\text { (j) } \\
\end{array}$ & $\begin{array}{l}\text { "eh } \\
(j)\end{array}$ & $\frac{P \Delta t}{T_{\text {eh }}}$ & $\begin{array}{l}T_{e} \\
(5)\end{array}$ & $\begin{array}{c}\text { Limiting hot } \\
\text { electron loss } \\
\text { orocess }\end{array}$ \\
\hline $\begin{array}{l}\text { Tandem mirru. } \\
\text { TMX-Upgrade }\end{array}$ & $4 \times 10^{12}$ & $a$ & 33 & री & a & 100 & 0.075 & $1.0 \times 10^{4}$ & 600 & 27 & O.OกA & Coulonib \\
\hline $\begin{array}{l}\text { Minimum-B mirrors } \\
\text { CIRCE }\end{array}$ & $3.5 \times 10^{11}$ & $2.2 \times 10^{17}$ & 100 & 2.5 & $?$ & 14 & $n . n ?$ & 780 & 21 & 13 & 0.48 & $?$ \\
\hline $\begin{array}{l}\text { Simple mirrors } \\
\text { TPM }\end{array}$ & $1 \times 10^{10}$ & $1 \times 10^{12}$ & 100 & 1 & "2" & 5 & 0.02 & 100 & 0.16 & 525 & 1.6 & Microinstabijity \\
\hline $\begin{array}{l}\text { Internal rings } \\
\text { SCL }\end{array}$ & $1 \times 10^{10}$ & $7 \times 10^{11}$ & 250 & 50 & "7ก" & 1 & 0.5 & 0.5 & 50 & 10 & 6.7 & $?$ \\
\hline
\end{tabular}

of iassumed equal to the hot-electron density. 
plasma. Next in efficiency is CIRCE with $8 \%$ of the applied energy stored in the plasma. This provides a benchmark regarding startup efficiency. Other minimum-B and simple mirrors were less efficient.

\subsection{DISCUSSION}

The big question is how to extend these conclusions to TMX(N). On one hand, TMX(N) has more control (neutral beams, ICRH, and gas box feed away from che ECRH power, etc.) so it does not rely on ECRH alone to produce the plasma. Ili uddition, TMX-Upgrade antennas only illuminate the plasma locally near cyclotron resonarice. On the other hand, any power not absorbed on one pass is not confined within a microwave cavity for subsequent absorption as in most previous experiments. Presentiy, ray tracing calculations point out the opposite concern, that the power may be absorbed at the plasma edge.

A second noteworthy comparison is that the $\operatorname{TMX}(N)$ hot-electron lifetimes are only $8 \mathrm{~ms}$, relative to several second theoretical values of other experiments. This means some loss processes may be less severe in $\operatorname{TMX}(\mathrm{N})$. For example, hot electrons need to be confined for $10^{6}$ bounces in $\operatorname{TMX}(\mathrm{N})$. Other experiments (typically shorter length machines and higher energy electrons) theoretically should have held the electrons for $10^{9}$ or more bounces. While previous experiments did not achieve the theoretically predicted electron confinf:ment, they did, however, confine electrons for more than the $10^{6}$ bounces required in $\operatorname{TM} \times(N)$.

Nonadiabaticity was a major loss process in several experiments. Nonadiabatic losses are not expected in $T M X(N)$ due to the higher magnetic field strength, larger magnet size, and lower projected mean-electron energy.

Several possibilities to either decrease the power requirements or increase the available heating power are listed in Table 5-3. The first two have no major cost impact but will reduce the operating parameters. The others would maintain performance with additional cost. 
Table 5-3. Possible options to reduce ECRH power requirements.

Option Consequence $\quad \begin{gathered}\text { Possible } \\ \text { gain }\end{gathered}$

Best suited for negative plug. gain

1. Reduce plasma radius in half Reduces central-cell radial confinement time

2. Reduce gperating density to Reduces radial nt. P1asma 4 $2 \times 10^{12} \mathrm{~cm}^{-3}$ more vulnerable to charge exchange loss

3. $5.0 \mathrm{~T}$ axicoil insert

May also help reduce required pump and central-cell beam current

4. Improve microwave transmission efficiency using large waveguide

No practical design available. Coupling efficiency may decrease

5. Double microwave power

Additional gyrotrons are 2 expensive

6. Supf ement heating with

Physics uncertainties $\underline{3}$ electron beans

Total possible gain $=576$ 
............

$-70-$ 


\section{SECTION 6 \\ MICROINSTABILITY}

(T. A. Casper, G. R. Smith, W. M. Nevins)

Success of the negative tandem mirror relies on our ability to generate stable, hot, mirror-confined electron distributions. Assuming MHD stability, we must still contend with electron miurostability since past hot-electron experiments have experienced very rapid (microsecond time scale) loss of confinement in the presence of instabilities near the electron-cyclatron frequency. Since this may result in a limit to the total energy and density of the hot electrons, it is imperative that we understand the nature of electron modes to which $\operatorname{TMX}(N)$ is susceptible, their stability, and their effects on confinement.

By electron microinstability, we refer to those modes which are spontaneously unstable near the electran-cyclotron frequency or its harmonics. Three such instabilities have been considered to date with varying degrees of detail; the upper-hybrid loss-cone (UHLC) mode, the whist 7er mode, and the anisotropy-driven mode. Since the naming coivention for these modes is not standard throughout the literature, we supply the following list of synonyms:

\begin{tabular}{|c|c|}
\hline Th is report & also used in literature \\
\hline $\begin{array}{l}\text { Upper hybrid loss cone } \\
\text { (electrostatic) }\end{array}$ & $\begin{array}{l}\text { 2-component (sometimes with } k_{\| 1} \neq 0 \\
\text { double-humped electron }\left(k_{\|}=0\right) \\
\text { double distribution }\end{array}$ \\
\hline Whistler (electromagnetic) & Electron cyclotron \\
\hline Timofeer (electrostatic) & $\begin{array}{l}\text { Harris } \\
\text { anisotropy-driven } \\
\text { half-harmonic }\end{array}$ \\
\hline
\end{tabular}

Note that both the whistler mode and the Timofeev flode are driven by the hot-electron anisotropy. To the present date, we have considered the UHLC-mode in greatest detail. After a brief initial study, we concluded it was the most likely to exist. Based on work presented here, and specifically, upon past experimental evijence, we now feel the whistler mode should be given more attention. We are still of the opinion that the Tim. feev node will not be a problem. 


\subsection{THEORETICAL REVIEW}

An early review of microinstabilities of ECRH plasmas was provided by Guest and Sigmar. 'The instabilities that led to the most stringent constraints or plasma parameters were the electromagnetic whistler instability and the electrostatic UHLC instability. Later work on the whistler instability has shown that relativistic effects are significantly stabilizing even for mean electron energies much less than the rest energy. ${ }^{2-4}$ The UHLC instability was the subject of a detailed study for plasma parameters appropriate to the earth's magnetosphere. 5

In this previous work ${ }^{1-5}$ it is found that the wavelengths associated with these high-frequency eiectron modes are short in comparison to typical macroscopic scale lengths of the plasma. Hence, requiring stability to these modes tends to constrain the electron distribution function rather than the configuration of an experimental plasma. Theoretical work naturally begins with the uniform-plasma approximation. Hot electrons have been treated using separable distributions such as a two-temperature Maxwellian to madel anisotropy and a distribution that vanishes at zero perpendicular velocity to model a loss cone. Cold electrons have been included in some work. We are presently developing a code ${ }^{6}$ (Section 6.4) to determine the stability boundaries for velocity distribution functions more relevant to tandem-mirror experiments.

From the review article by Guest and Sigmar 1 , we obtain the stability boundaries shown in Fig. 6-1 as a function of cold-electron density, $\omega_{p c}^{2} / \Omega^{2}$ where $\omega_{p}$ is the electron plasma frequency for the species indicated and $\Omega$ is the electron-cyclotron frequency, versis inverse anisotropy, $T_{\| l} / T_{1}$ of the hot-electron distribution (isotropy implies $T_{\|}=T_{\perp}=T_{\text {eh }}$. It is readily apparent that a stable band of cold-electron density $n_{e c}$ exists. At high $n_{e c}$ the plasma is unstable to the anisotropy driven mode if $T_{I I} / T_{L} \leq 0.3$ whereas it is unstable to the UHLC-mode at low nec. Note that the UHLC-mode is stabilized by increasing the anisctropy (decreasing $T_{\| 1}\left(T_{1}\right.$ ). The analys is used was valid only in the high $n_{\mathrm{ec}}$ limit $\left(\omega_{\mathrm{pc}}^{2} \gg \omega_{\mathrm{ph}}^{2}\right)$ where wave properties are determined by the cold electrons. 


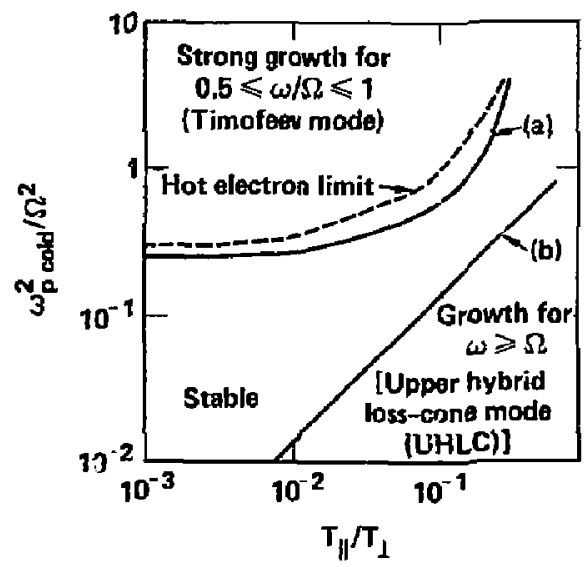

Figure 6-1. Critical cold plasma densities vs temperature anisotropy; from G. E. Guest and D. J. Signar, Nuc\}, Fusion 11, 151 (1971). 
C.ncentrating now on the UHLC instability in the high $\mathrm{n}_{\mathrm{ec}} 1$ imit where the wave properties are determined from the cold plasma Jispersion rotation, i.e., the cold electrons merely serve to support the upper hybrid irave. The instability is driven by the loss cone dist-ribution of the hot mirror-confined electrons. Thus, at sufficiently high values of $n_{e c}$, marginal stability is achieved by filling the loss cone. This transition is represented by the stability boundary (b) in Fig. 6-1. At very low cold electron density, i.e., beyond the range of the Guest and Sigmar analysis, 1 stability again be achieved when the cold electron density becomes insufficient to support the wave. An analysis by Young et al. ${ }^{7}$ shows that stability is achieved at $\mathrm{n}_{\mathrm{ec}}=0$ provided the loss-cone distributions are not too severe. For very steep loss-cone distributions (large af/av $)$ instability occurs even in limit $n_{\text {ec }}=0$.

A boundary for the convective-absolute transition of the UHLC instability in the limit of isotropic hot electrons is provided by Guest and Sigmar, Fig. 6-2, as a function of $n_{\mathrm{ec}}$ versus $\mathrm{n}_{\mathrm{eh}}\left(\omega_{\mathrm{pc}}^{2} / \Omega^{2}\right.$ versus $\left.\omega_{\mathrm{ph}}^{2} / \Omega^{2}\right)$. Using the TMX(N) density profiles, Fig. 6-3, we are able to display in Fig. 6-2 a stability trajectory of the axial density variation assuming $T_{\mid I} / T_{1}=0.3$. The conclusion drawn from this data is that $T M X(N)$ is absolutely unstable over most its axial extent towards the central cell. This conclusion, however, is overly pessimistic since increased anisotropy aids stability of the UHLC-mode. Plotting this axial variation at $T_{11} / T_{1}=0.3$ in Fig. 6-1 indicates that $\operatorname{TMX}(N)$ lies close to the stability boundary; a result of the stabilizing effect of anisotropy. Additional stabilizing effects will result from nonzero temperature cold electrons and finite geometry effects (Section 6.3). These results do, however, indicate that caution must be exercised in regards to the contial of the density and temperature of cold electrons.

A numerical study 5 of the UHLC-mode has found it to be unstable (temporal growth) over a wide range of density and temperature ratios, $n_{\mathrm{ec}} / \mathrm{n}_{\mathrm{eh}}$ and $\mathrm{T}_{\text {ec }} / \mathrm{T}_{\text {eh }}$ Again, this analysis was completed for an isotropic hot-electron distribution and therefore tends to present a pessimistic view of UHLC stability. On the other hand, the loss cone was weak and this tends to be optimistic. Reference 5 indicates the most important result is that the cold electron temperature controls spatial amplification. For $T_{e c}=0$ the instability is convective with a very large growth rate. In the range $0<T_{y c} / T_{\text {eh }}<0.01$, finite $T_{e c}$ induces a nonconvective (absolute) instability 


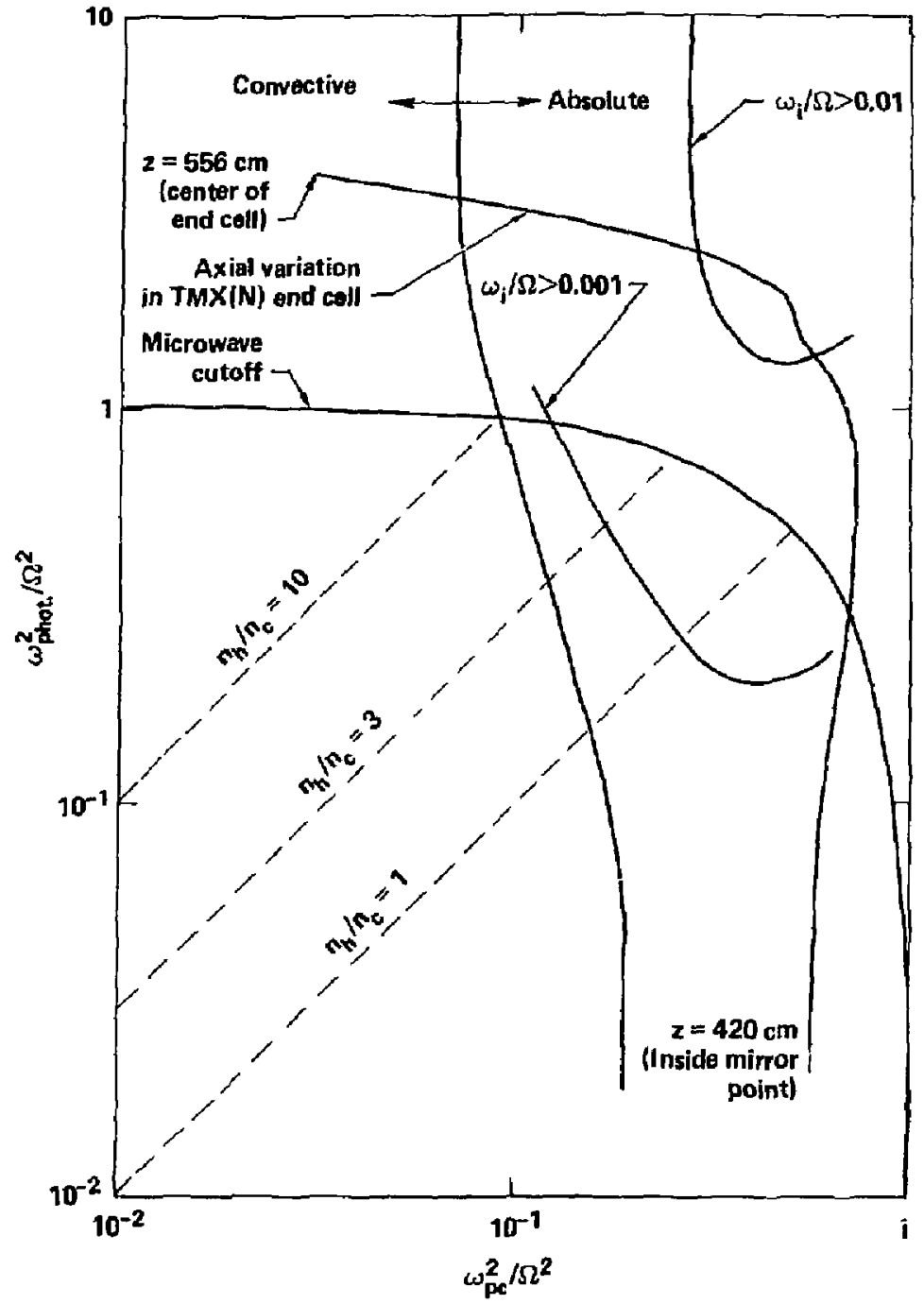

Figure 6-2. Axial density variation of $T M X(N)$ end cell flatbed relative to the loss-cone mode growth characteristics from G. E. Guest and D. J. Signar, Nucl. Fusion 11. 151 (1971). 


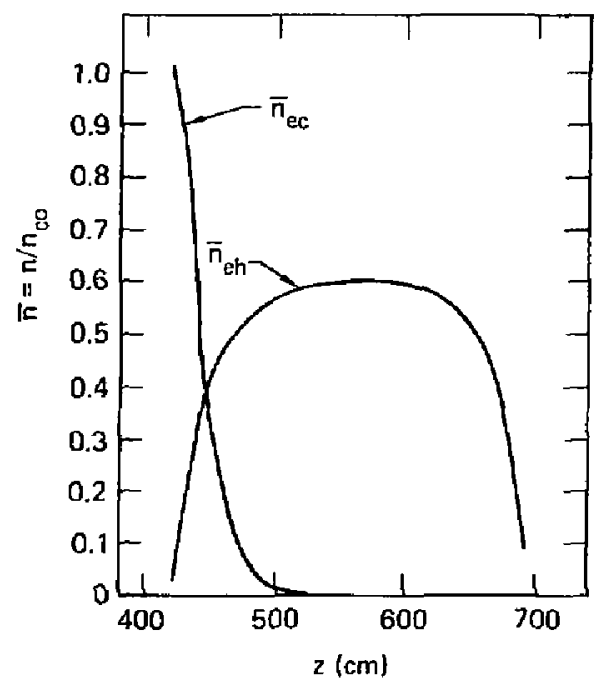

Figure 6-3. Normalized axial density variation. 
which persists until $T_{e c} / T_{e h}=0.05$. At this temperature ratio, the nonconvective instability disappears. These results may be substantially altered if the cold electrons are allowed to be spatialiy nonuniform as is the case in $T M X(N)$. Additional results of this numerical study pertinent to TMK(N) operation are listed here:

- $n_{e c}$ controls the unstable frequency (agrees with Young ${ }^{7}$ et al., where the wave frequency $w$ approaches $\Omega$ when $n_{\mathrm{ec}}+0$ with a severe loss cone and $\omega$ appraaches $2 \Omega$ for a gentle loss cone in the presence of culd electrons).

- Stability results for large $n_{\mathrm{ec}} / \mathrm{n}_{\text {eh }}$ which may be significant near the inner-mirror location.

- Filling tine hot-electron loss cone reduces $T_{e c}$ for the absolute-colvective transition but does not remove the absolute property for sinall $\mathrm{T}_{\mathrm{ec}} / \mathrm{T}_{\mathrm{eh}}$.

- Perpendicular heating of cold electrons can change the instability from absolute to convective.

- Limitation of the convective growth rate $c$ an be achieved by a combination of cold-electron diffusion and hot-electron loss. Without some other control on the loss cone, stabilization by hot-electron loss would place additional demands on the ECRH and electron feed systems.

These results indicate that if the UHLC instability is present in, it will be detrimental to confinement. Since the instability searches for stability by heating cold electrons and expelling hat electrons (filling loss cone and reducing positive slope of the electron velocity distribution), either a density 1 imit would be reached or the loss rate would be enhanced. Additional ECRH power may be necessary to replenish the enhanced hot-electron loss or neat the cold electrons to achieve stability. It is entirely possible that $\operatorname{TMX}(N)$ may have to operate with a finite level of residuai fluctuations as would similarly be true of the thermal barrier in TMX(P). This is equivalent to the operation of single mirrors and simple tandem mirrors with a finite level of ion-cyclotron fluctuations as was done in the past.

The whistler mode has not yet been studied in as great detail as the UHLC mode. A few points are worthy of notice, however. The whistler mode 8 is intrinsically electromagnetic in character and is driven by the anisotropy of the hot electrons. It has been shown to be more unstable ${ }^{9}$ (larger growth 
rate) in the presence of cold electrons. The absolute-convective boundary, $10,1 T$ is given by $T_{\| 1} / T_{1}=\sqrt{9 B_{1} / 27}$ and $r c$ growth is possible if $T_{\|} / T_{\perp}>\sqrt{\beta_{1} / 2}$. At $T_{e h}=50 \mathrm{keV}$ and $\eta_{e h}=5 \times 10^{12}$, TMX(N) with $T_{\|} / T_{\perp}=0.3$ would appear to be unstable to the whistler mode. Axial variations in all parameters need to be studied in detail to determine the true stability to this mode.

\subsection{EXPERIMENTAL REVIEH}

The preceding discussion indicates that $T M X(N)$ siands a reasonable chance of being unstabia to electron modes since its parameters approach or exceed the theoretical estimates for marginal stahility. With this in mind, a review of past mirror-confined hot-electron experiments $12-21$ was jone to determine their agreement with the theory availahle and what affects on confinement have been observed. There is a fairly substantial number of past experiments susceptible to all three instabilities discussed. In Tabie 6-1 we present a short sumary of experimental conditions present during instability. From the published 1iterature, it was generally impossible to determine the shape of the plasma, i.e., ring versus disc. Both ELMO ${ }^{12}$ and TPM ${ }^{13}$ were ring-shaped and Table Top $\mathrm{IV}^{14}$ was peaked on axis (probabl $j$ true for a11 pulsed-field compression experiments). Three techniques were used to create and heat the electrons; beam plasma interaction ${ }^{15}, \mathrm{ECRH}^{12,73,16-18,21}$, and pulsed-field compression 14,19,20. The gross parameter ranges of operation have been plotted on the stability didgram for the uniform cold electron model. Fig. 6-4. In most experiments, the cold electron density was indirectly determined from ionization by hat electrons. The anisotropy range generally has been calculated from the mirror ratio and plasma length. He aiso plot the whistler unstable experiments $13,18-20$ for comparison (the stability curve really has nothing to do with the whistler mode).

There seems to be fairly good agreement between the theoretical stability boundaries and the location of the unstable experiments. For ail data presented, except for CIRCE ${ }^{21}, T_{e c} / T_{e h}<i \%$ and the assumption $T_{e c} \approx 0$ is reasonable. For the CIRCE experiment, $T_{e c}<5 \mathrm{keV}$ was reported. The UHL: boundary plotted for $T_{\mathrm{ec}}=0$ will shift to lower $\mathrm{wpc}_{\mathrm{pc}}^{2} / \Omega^{2}$ values for $T_{e c}>0$ and therefore enhance the stable region. The "stabie" CIRCE experiment 
Table 6-1. Sumlary of conditions present during instabitity.

\begin{tabular}{|c|c|c|c|c|c|c|c|c|c|c|c|c|}
\hline No. & $\begin{array}{c}\text { inst abtisty } \\
\text { name }\end{array}$ & $\begin{array}{l}\text { Ref. } \\
\text { nn. }\end{array}$ & $\omega_{p h}^{2} n^{2}$ & $w_{\mathrm{pr}}^{?} n^{?}$ & $r=T_{\operatorname{lnh}} /{ }^{\top} \mathrm{h}$ & $\frac{B_{0}}{(k-5)}$ & $\frac{B_{m}}{n}$ & ${ }^{\circ} \mathrm{ceo}$ & $f_{z t} \operatorname{cen}$ & $\left(\mathrm{cm}^{\mathrm{n}^{-3}}\right)$ & $\begin{array}{c}n_{e c} \\
\left(\mathrm{~cm}^{-3}\right)\end{array}$ & $n_{e c} / n_{e a}$ \\
\hline 1 & $\pi$ & 15 & $\begin{array}{l}0.47 \\
0.14\end{array}$ & $\begin{array}{l}33.5 \\
10.1\end{array}$ & $0.1-0.7$ & 0.0 & 0.7 & 1.1 & 2.0 & $7 \times 10^{9}$ & $5 \times 10^{11}$ & 71 \\
\hline 2 & T2 (ELMO) & 12 & $\begin{array}{l}0.76 \\
0.03\end{array}$ & $\begin{array}{l}0.16 \\
0 . n 3\end{array}$ & - & 9 & 17.2 & $72 . a$ & ¿e.? & $1 \times 1017$ & $1 \times 101 ?$ & 1 \\
\hline 3 & $T 3$ & 16 & $\begin{array}{l}n . ? \\
0.8\end{array}$ & $\begin{array}{l}n .2 n \\
0.16\end{array}$ & 0.167 & 0.7 & 1.1 & 2.0 & 3.1 & $9 \times$ & $2 \times 10^{10}$ & 2 \\
\hline 4 & $\begin{array}{l}\text { UHLC-1 } \\
\text { (INTEREM) }\end{array}$ & 17 & $\begin{array}{l}0.11 \\
0.07\end{array}$ & $\begin{array}{l}0.199-n .16 \\
0.06-n .10\end{array}$ & 0.1 & 3 & 3.3 & 8.4 & $: 0 . \hbar$ & ו 1 & $0.8-1.4 \times 10^{11}$ & 1 \\
\hline 5 & $\begin{array}{l}\text { UHLC-2 } \\
\text { (TT IV) }\end{array}$ & 14 & $2.5^{0.01} \times 10^{-3}$ & - & n.? & in & 20 & $28 . \Gamma$ & 56.0 & $1 \times 1 \times 1$ & - & - \\
\hline 6 & (5ame as 5 ) & (14) & $2.5 \times 10 t$ & $\begin{array}{l}4 \times 10-3-0,01 \\
I=7.5 \times 10^{3}\end{array}$ & 0.2 & in & 20 & $28, n$ & 56.0 & $1 \times 10^{11}$ & $0.4=1 \times 10^{1} 1_{0.4}$ & $4-1$ \\
\hline$?$ & WI (TFM) & 13 & $\begin{array}{l}0.81-8.07 \\
6.5 \times 10^{-4}-0.01\end{array}$ & $\begin{array}{l}0.7-7.01 \\
0.06-0.65\end{array}$ & - & 1.8 & 4.0 & 3.4 & 11.2 & $0.1-1 \times 10^{18}$ & $0.7-7 \times 0_{0} 0^{12}$ & $10-1000$ \\
\hline 8 & (Same as 7 ) & (13) & $\begin{array}{l}0.07 \\
0.07\end{array}$ & $\begin{array}{l}0.17 \\
0.01\end{array}$ & 0.07 & 1.7 & 4.0 & 3.4 & 11.2 & $1 \times 10^{10}$ & $1 \times 10^{10}$ & $1-10$ \\
\hline 9 & W? & 18 & $a_{\times 10-3}^{0.01}$ & $\begin{array}{l}0.06 \\
0.04\end{array}$ & 0.2 & $3 . n$ & 3.6 & 8.4 & $10 . ?$ & $5 \times 10^{9}$ & $5 \times 10^{10}$ & 10 \\
\hline in & W3 and $T 4$ & 19 & $\begin{array}{l}0.03-1.6 \\
0.01=0.72\end{array}$ & $\begin{array}{l}0.13=6.5 \\
0.03=1.4\end{array}$ & $0.1-0.7$ & $8-18$ & 12.77 & $\begin{array}{l}3.4= \\
33.6=\end{array}$ & $\begin{array}{l}-50.4 \\
-75.5\end{array}$ & $0.1-1 \times 10^{13}$ & $\begin{array}{l}0.4-4 \times 10^{13} \\
0.2=3 \times 10^{13}\end{array}$ & 4.3 \\
\hline 11 & (Same as 7 & (13) & $\begin{array}{l}0.07 \\
0.01\end{array}$ & $\begin{array}{l}0.70 \\
0.0 \mathrm{~K}\end{array}$ & - & $1 . ?$ & 4.0 & 3.0 & 11.2 & $1 \times 10^{10}$ & $1 \times 10^{11}$ & 10 \\
\hline$! 2$ & 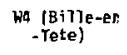 & 20 & $\begin{array}{l}0.05-0.37 \\
0.07-0.12\end{array}$ & $\begin{array}{l}0.25-3.7 \\
0.09-1.22\end{array}$ & 0.73 & $\begin{array}{l}3.5= \\
2.5=\end{array}$ & $\begin{array}{l}2.2 \\
3.7\end{array}$ & $3.2=$ & 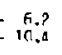 & $2.5-8 \times 10^{10}$ & $1.25-8 \times 10^{17}$ & $5-10$ \\
\hline 3 & $\begin{array}{l}\text { "Stable" } \\
\text { CIRCE }\end{array}$ & ? ? & $\begin{array}{l}0.49 \\
0.09\end{array}$ & $\begin{array}{l}0.31 \\
0.06\end{array}$ & - & 2.7 & $6 . ?$ & 7.6 & 17.4 & $3.5 \times 10^{31}$ & $7.2 \times 10^{11}$ & 0.37 \\
\hline
\end{tabular}




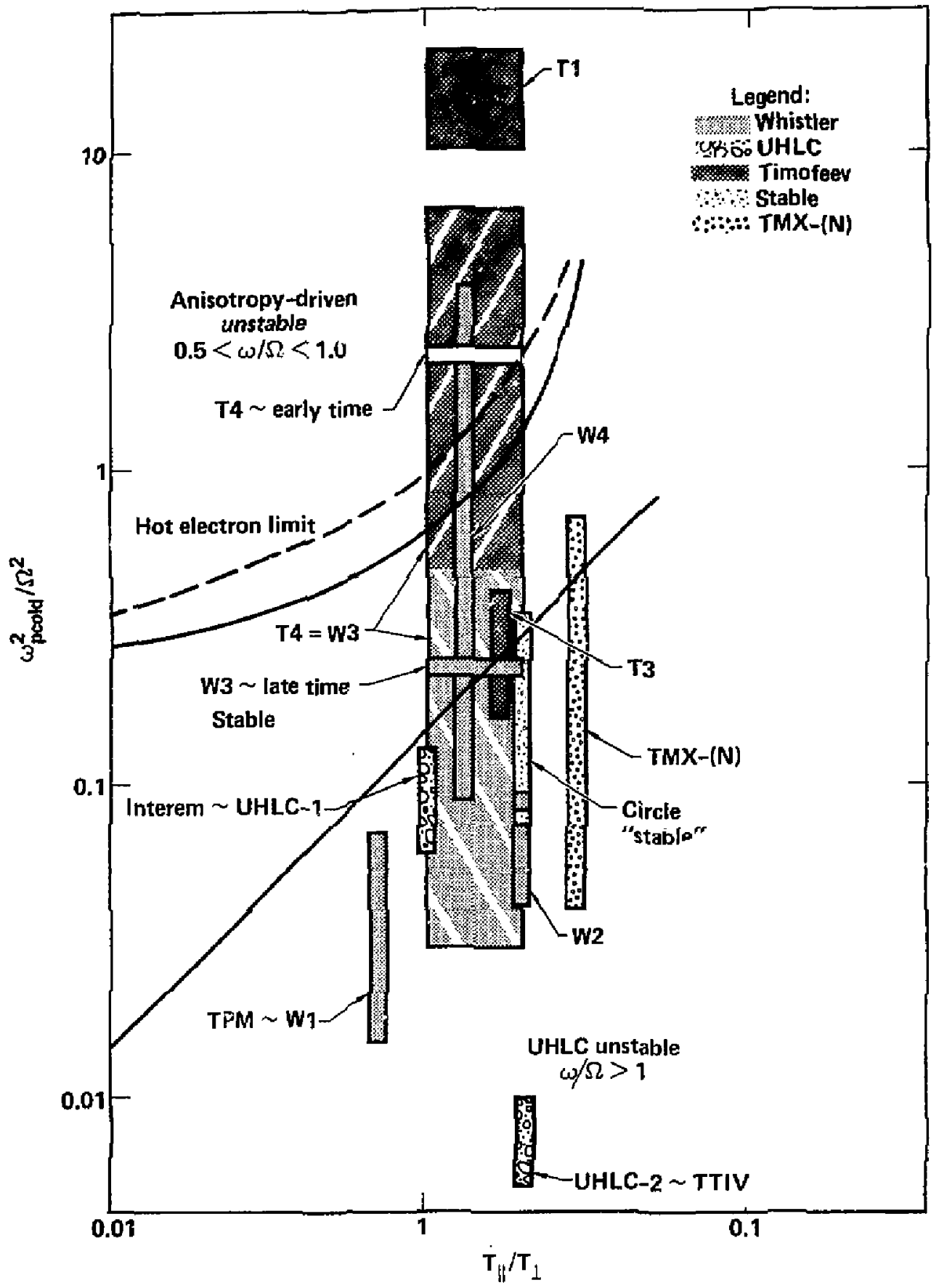

Figure 6-4. Operating ranges of past experiments for electron microstability. 
may very well lie within the stable band. It is intere:iting to note that for CIRCE at $T_{e c}=5 \mathrm{keV}, \mathrm{T}_{e c} / \mathrm{T}_{\mathrm{eh}}=5 \%$ which is the ratio predicted for stability by the previously discussed numerical study ${ }^{5}$. While no evidence for gross microinstability was reported on CIRCE, the experimenta!ly determined electron loss rate was 23 times "classical" and no explanation was given. The classical calculation included only scattering from a single electron species in a square-well magnetic field. No effects were included to model either the two electron species, cold and hat, or the spatially varying density and magnetic field profiles. These effects can significantly increase the classical loss processes.

In all cases, the instability identity was determined by the frequency of microwave emission detected. For most experiments, the microwave emission correlated we1T with axial bursts of hot electrons and in a few cases radial losses were also observed. The rapid loss of confinement was evidenced by a drop in plasma diamagnetism with up to $80 \%$ loss of hot electrons reported. In instances where the plasma is being driven (pulse compression) during the instabjlity, multiple bursts were observed indicating a quasi-steady state may be achieved similar to that experienced in hot-ion positive tandems. The important lesson to be learned is that a drastic reduction in hot-electron conf inement can occur over a very short time scale, $\approx 1 \mu \mathrm{sec}$.

In past experiments, stabjlity has been achieved in two ways. One is by altering the velocity distribution of electrons either self-consistently by the instability creating and heating cold electrons or diffusing hot electrons or both or by supplying additional cold electrons. "Upper off-resonant heating" has also been used to provide stability ${ }^{17}$ though no detailed explanation for how it works has been presented. Note that the expe:inentally observed effects of the instability are qualitatively consistent witi the numerically predicted quasilinear s aturation effects,

\subsection{FINITE GEOMETRY STABILIZING EFFECTS ON UHLC INSTABILITY}

After reviewing stability in a uniform plasma, the effects of spatial inhomogeneity was studied. ${ }^{22}$ We again use the 1 imit where wave propagation is determined by the cold plasma dispersion, $w(k)$. Since the parallel group velocity is generaliy larger than the perpendicular group velocity, the axial eigenmode problem was considered. Fron the assumed axial profiles of magnetic 
field and cold electron density in the end cell, the axial variation of $k_{11}^{2}$ is obtained, Fig. 6-5. Wave packets can be born at $\omega=\Omega$ in the unstable region, $k_{11}^{2}<0$. At worst, an unstable wave packet can undergo one reflection before being absorbed at $\omega=\Omega$. Thus, no axial eigenmodes can exist and the instability can only be convective. This implies that the more serious absolute UHLC instability should not occur. The reason for the enhanced stability of the end cell stems from the monotonically decreasing nature of the axial cold electron density profile. Should this variation be altered, i.e., if the cold electron density peaks at the center of the end cell, then TMX(N) would be expected to be UHLC absolutely unstable and perhaps exhibit. those unstable characteristics of past experiments.

It is evident that much work needs to be done concerning both the theoretical stability of the TMX(N) plasma and its relation to past experiments. To improve our understanding of hot-electror stability pertaining to negative tandems (and thermal barriers) we are developing a code (similar to that used for hot-ion distributions) which will determine stability boundaries for distributions not constrained to be separab?e in $\left(v_{1}, v_{11}\right)$-space and therefore more representative of Fokks.-Planck calculations. Initially, only the spatially uniform case will be considered.

\subsection{STABILITY CODE}

Fokker-Planck studies ${ }^{23}$ have fou d distributions like the one shown in Fig. 6-6. From the contour plot [Fig. 6-6(a)] we see, first, that the anisotropy (disparity between mean perpendicular and parallel energies) is moderate. Therefore, instabilities requiring extreme anisotropy are not expected; the whistler and UHLC instabilities may occur, however, in a p?asila with such a distribution. Also, we notice that the distribution is not separable in $v_{1}$ and $v_{11}$, i.e., $f(\underline{v})=f_{1}\left(v_{1}\right) f_{11}\left(v_{11}\right)$. Separable distributions, like those used in Ref. 1, have constant-density contours in velocity space that are generally elliptical with the major and minor axes aligned with the $v_{\perp}$ and $v_{\| l}$ axes. It is clear from $;$ ig. 6-6(a) that the Fokker-Planck distribution is not separable. Separable distributions have been used in most previous studies; exceptions are the whistler-instability studies of Scharer ${ }^{8}$ and Jacquinot and Leloup. 2 


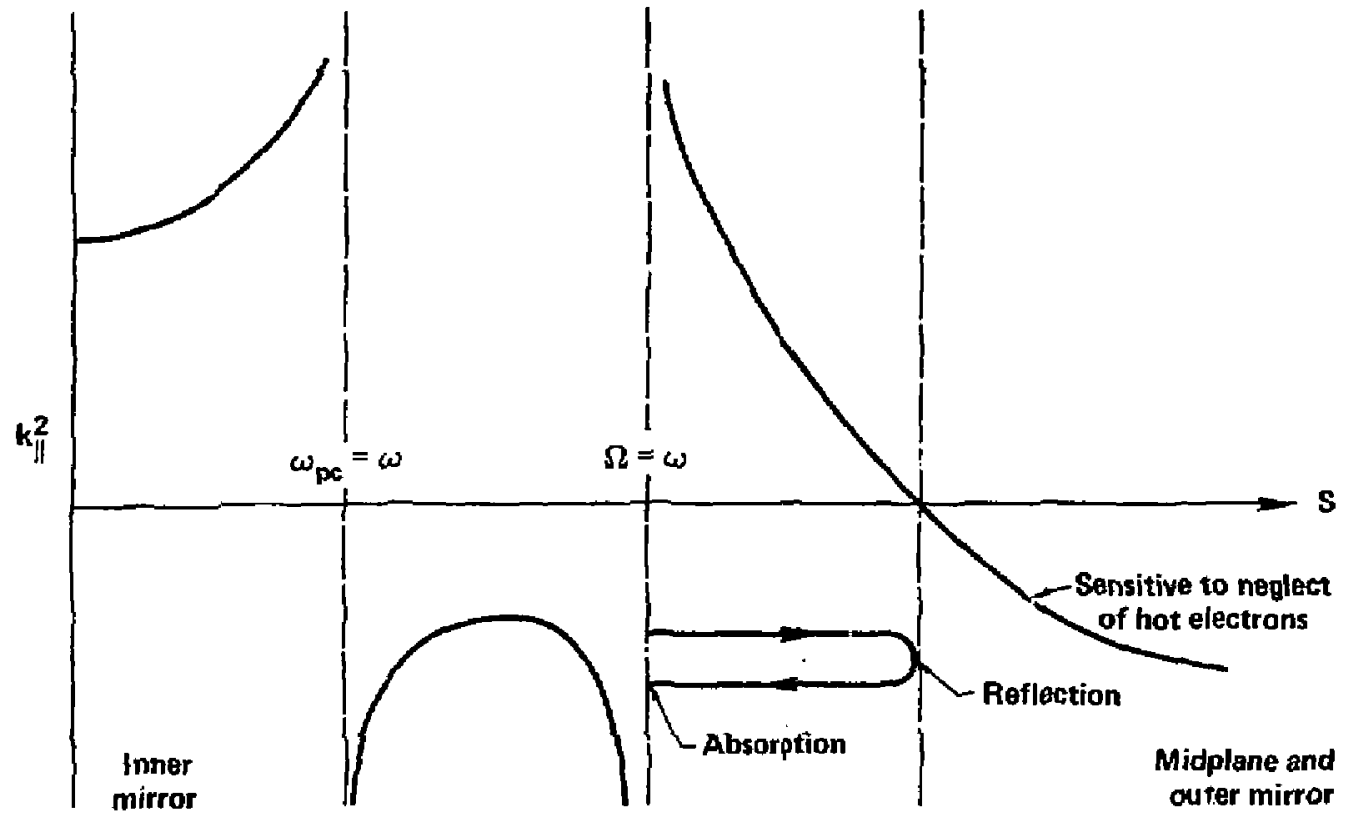

Figure 6-5. Parallel wave number vs axial position for typical TMX(N) profiles. 

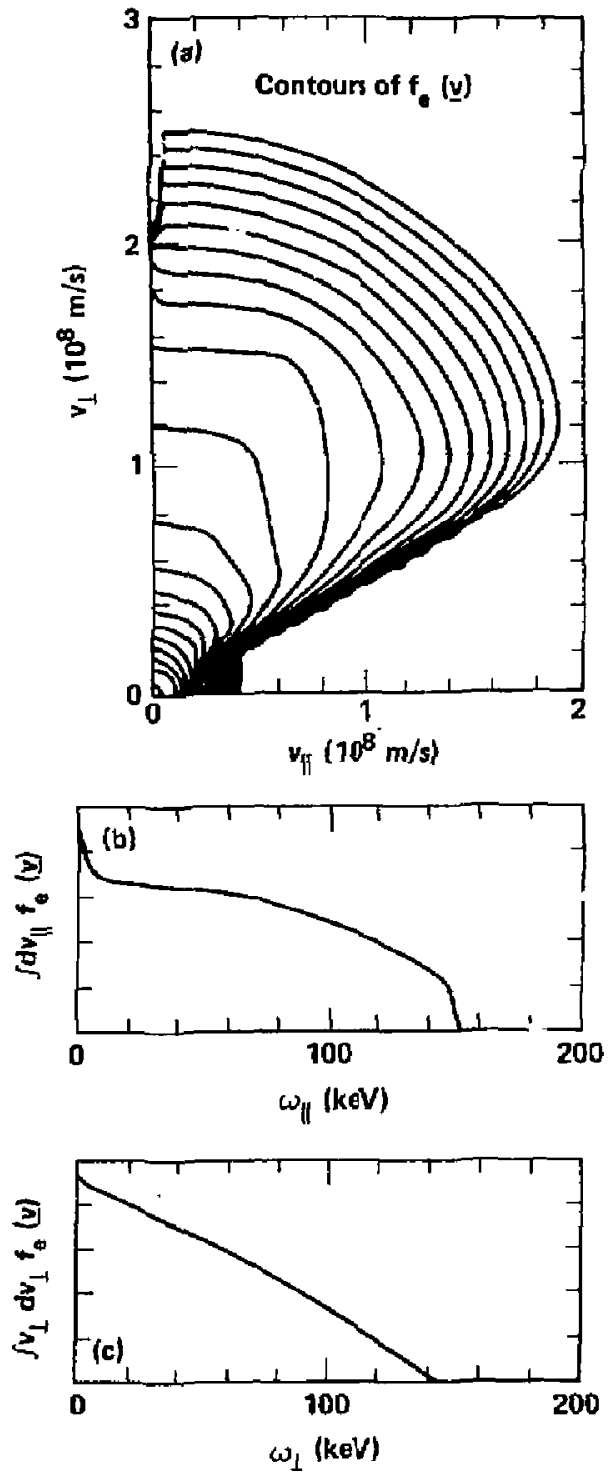

Figure 6-6. Electron distribution found in Fokker-Planck studies of the FCRH : lasma in the TMX-Upgrade Experiment. The total electron density is given py $\omega_{p}^{2} / \Omega^{2}=1.55$. (a) Contcurs of constant distribution function. Contours are logarithmically spaced with a factor of two separating adjacent contours. (b) and (c) Distributions integrated over paralie? and perpendicular velocity, respecti tiy. The vertical scale is logarithmic, covering five decades. 
Modeling our Fokker-Planck distribution by a sr parable distribution would lead to erroneous conclusions about microstability. This is apparent when we compare Figs. 6-6(b) and 6-6(c) with Fig. 6-6(a). If $f_{\perp}\left(v_{1}\right)$ is chosen to approximate the distribution shown in Fig. 6-6(b) and $f_{\| 1}\left(v_{\| l}\right)$ is chosen to model the distribution of Fig. 6-6(c), then the separable model distribution, $\tilde{f}_{1}(\underline{v})=f_{1}\left(v_{1}\right) f_{\| f}\left(v_{\|}\right)$would not have a loss cone. Hence, only anisotropy-driven modes, like the whistler, would appear with positive growth rates in our stability analys is. Clearly such a mr-s1 would greatly overestimate the stability of the actual electron distribution to modes, like the UHLC mode, that are driven by the loss cone.

The Fokker-Planck distribution in Fig. 6-6(a) cleariy has a loss cone. Since the average of $f(\underline{v})$ over parallel velocity is a monotonically decreasing function oi perpendicular energy, $W_{1}$, this loss cone cannot drive modes with $k_{\|}=0$. However, modes with finite $k_{\|}$can be driven by this loss cone since the resanant particles [i.e. those particles with $v_{11}=(\omega-n \Omega) / k_{n} ; n$ is the harmonic number] can be localized at a parallel velocity where there is an inverted distribution in perpendicular velocity. Hence, we expect that distribution functions like the Fokker-Planck distribution shown in Fig. 6-6(a) can drive the UHLC mode.

We model fokker-Planck distributions like that in Fig. 6-6 by superposing a number $N$ of electron "species" with various parameters:

$$
f(\underline{v})={ }_{s=1}^{N} f_{s}(\underline{v}) \text {. }
$$

Each species has a separable distribution

$$
f_{s}(\underline{v})=\frac{n_{s}}{\pi^{3 / 2} \alpha^{2}, s \ell_{s} ! \alpha_{11, s}}\left(\frac{v_{1}}{\alpha_{1, s}}\right)^{2 l_{s}} \exp \left(-\frac{v_{1}^{2}}{\alpha_{1, s}^{2}}-\frac{v_{11}^{2}}{\alpha_{11, s}^{2}}\right) .
$$

By appropriate choices of the densities $n_{s}$, thermal speeds $\alpha_{1, s}$ and $\alpha_{11, s}$, and indices $\ell_{s}$, we achieve a good reproduction of Fig. $6-6$ as shown in Fig. 6-7.

Superposing separable distributions to model a nonseparable distribution allows us to avoid numerical velocity-space integrations, a considerable computational advantage. Distribution (2) is precisely that used by Callen and Guest, ${ }^{24}$ who provided a 1$]$ tre formulae neces:ary for numerical 

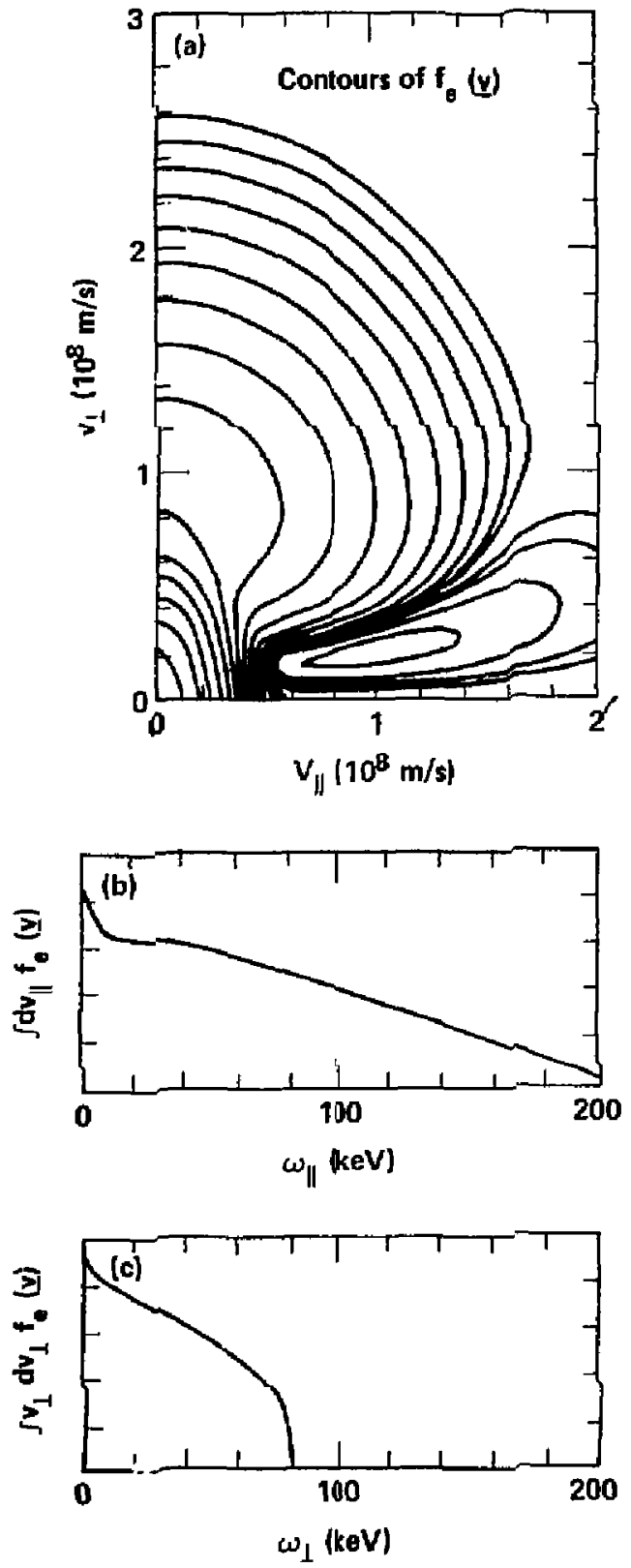

Figure 6-7. Model electron distribution formed by superposing five separable distributions (2). The piasma frequencies $\mu_{p}$, perpendicular temperatures

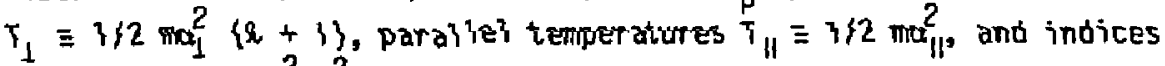
$\ell$ are given by $\omega_{p}^{2} / \Omega^{2}=0.35,0.5,-0.02,0.025$, and $-0.01, T_{1}=3,40,12,12$, and $4 \mathrm{keV}, T_{11} / T_{\perp}=0.3,0.35,4,2$, and 6 , and $\ell=0,1,1,1$, and $i$. 
calculation of the dielectric function $D(\omega, \underline{k})=\operatorname{det}[D(\omega, \underline{k})]$, whose roots describe the small amplitude waves of a collisionless plasma in a uniform magnetic field, $\underline{B}_{0}$.

We have written a code which solves this dispersion relation numerically. we allow for an electron distribution function in the form of Eq. (1), i.e., an arbitrary superposition of separable distributions as given by Eq. (2). For results presented here, the index $\&$ is limited to the values 0 (a bi-Maxwellian) and $f$ (a "loss-cone" distribution). We solve the full electromagnetic dispersion relation for wayes that propagate in an arbitrary direction with respect to $\theta_{0}$ in a uniform, non-relativistic plasma. Some results from this code will now be described.

ror the model distribution shown in Fig. 6-7 we have plotted contours in the $k$ plane of the temporal growth rates, Im $\omega$, of the whistler and UHLC instabilities. For the whistler (Fig. 6-8) the maximum growth rate occurs at $k_{1}=0$ and $k_{\| 1} c / \Omega=1.18$ where $\omega / \Omega=0.57+i 0.013$. Doubling the total electron density without changing the shape of the distribution (Fig. 6-7) shifts the maximum growth rate to $k_{||} c / \Omega=1.45$, where $\omega / \Omega=0.55+i 0.027$. This increase in $k_{\|}$is not unexpected since, in the $T_{\|}=0$ approximation, the whistler dispersion relation depends on $k_{11}$ only through the combination $\mathrm{k}_{\mathrm{II}} \mathrm{c} / \omega_{\mathrm{pe}}$.

The :emporal growth rates of the UHLC mode are shown in Fig. 6-9. We find that the maximum growth rate occurs at $k_{1} c / \Omega=9.2$ and $k_{11} / k_{1}=0.048$, where $\omega / \Omega=1.09+j 0.0046$. These values of $k_{1}$, and $k_{1}$ for the most unstable mode are in line with estimates presented by Guest and Sigmar.' Note that the growth rate of the UHLC mode goes to zero as $k_{\|}$goes to zero. This behavior follows from our earlier discussion of the Fokker-planck distribution.

When we double the total electron density, we find that the wave yector that maximizes the growth rate shifts to $k c / \Omega=10.04$ and $k_{\| /} / k_{1}=0.072$. where the frequency is given by $\omega / \Omega=1.16+i 0.0066$. 


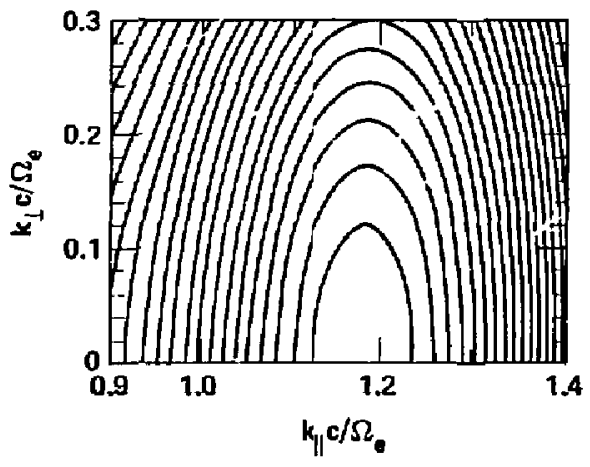

Figure 6-8. Temporal growth rate of the whistler instability for the electron distribution shown in Fig. 6-2. The interval between adjacent contours is $0.00063 \Omega$. 


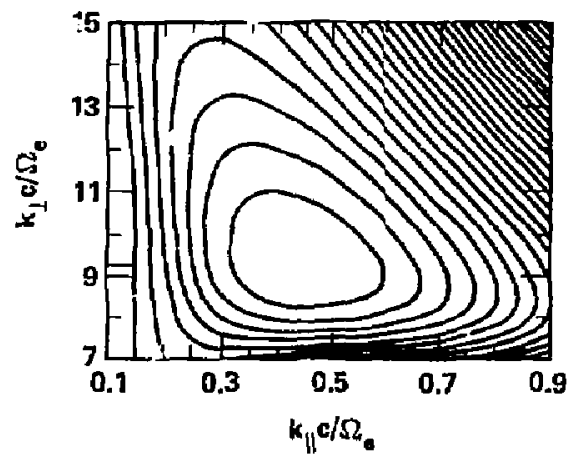

Figure 6-9. Temporal a tn rate of the upper-hybrid loss-cone instability for the electron distri. ion shown in Fig. 6-2. Solid contours give Im $\omega \geq 0$, dotted contours given im $\omega<0$. The interval between adjacent contours is $5.76 \times 10^{-4} \Omega$. 


\subsection{SUMMARY}

The negative tanden configuration for TMYX-Upgrade may be susceptible to microinstabilities near the electron-cyclotron frequency. The upper-hybrid loss-cone mode at $\omega \geq \Omega$ is driven by the loss-cone feature of the mirror-confined hot electrons whereas the whistler mode at $\omega \leq \Omega$ is driven by the anisotropy. Both are expected to be more unstable in the presence of a 1 imited supply of cold electrons and may result in an unstable band of cold electron density. Should $\operatorname{TMX}(N)$ be unstable to these modes, rapid, transient loss of confinement can be expected on microsecond time scales as evidenced by bursts of microwave emission correlated with axial and perheps radial dumps of hot electrons and a reduction in plasma diamagnetism. Since these instabilities are driven by the velocity-space distribution, further analysis using a stabiliiy code employing the more representative distributions produced by Fokker-Planck results is required to better assess hot-electron microstability in the presence of a uniform cold electron backgraund. Finite geometry effects, i.e., axial magnet ic field and density variations may render the upper hybrid loss-cone mode merely convectively unstable.

It should be recognized that, even in the presence of unstable oscillation, past experiments have reached densities and temperatures close to our present design objectives. It is believed that we can reach the required parameters, possibly at the experıses of additional ECRH power to overcome enhanced instability-produced losses. It should silso be recognized that some hot-electron experiments appear to be stable, notably EBT-like rings, 12 CIRCE, ${ }^{21}$ and the mirror experiment at the University of Wisconsin ${ }^{18}$ with heating off the midplane. 
SECTION 7

LOW FREQUENCY STABILITY

(O. E. Baldwin)

The stability to low frenuency, MHD-like modes under the condition of negative plug operation might be different from that under posit.: plug operation. At issue is the manner and degres to which the fast orifting, magnetically conf ined hot electrons can participate in the low frequency wave. The pressure in the minimum-B plug due to sloshing ions that are present in positive plug operation has a stabilizing contribution that is not present in negative plug operation. However, changes in other plug parameter: roughly compensate for this by increasing the pressure of trapped ions, so that at least interchange stability is predicted.

The physics underlying the response of the hot eiectrons is very similar to that of the hot electrons in EBT, particularly in the presence of a finite core $\beta$ that has not been achieved experimentally. At sufficientily low core $B$, here defined as $\beta_{w}$ (for $\beta$-warm), the hot electrons are rigid. The core plasma maves in the magnetic well enhanced by the hot-electron beta, $\beta_{h}$. Provided $\beta_{h} \gg 2 r_{p} / R_{c}$, which is aiways satisfied, with $r_{p}=$ plug rarius and $R_{c}=p$ ?. $g$ radius of curvature, the appropriate contribution of the plug to the interchange weighting is

$$
\int_{p l u g} d 1 \frac{{ }^{B}{ }^{\beta} h}{2 r_{p}^{2}} .
$$

If the hot electrons are treated as an MHD fluid, the corresponding weighting would be

$$
\int_{p 1 \text { ug }} d \frac{B_{h}}{r_{p} R_{c}} \text {. }
$$

Achieving an equivalent plug weighting for the warm ions in the self well of the hot electrons of at least as much as would have been obtained for the hot e lectrons counted as an MHD fluid requires

$$
\beta_{W} \geq \frac{2 r_{p}}{R_{c}} \text {. }
$$


Vaiues of $3^{3}$ in this range have not been achieved in EBT. This value, $B_{w}=2 r_{p} / R_{c}$, is the critical core $B$, predicted by Van Bam and Lee $l$ above wich the hot electrons begin to behave as an Miad fluid in spite of their $f a s t$ magnetic drifts. In EBT, the hat electrons would then be unstable in the negative magnetic curvature. In TMX-U, they would add their pressura to the favorable presslire weighting in the positive curvature of the plug.

A functional form that bridges these two limits is given by

$$
\int_{p \text { 1ug }} \frac{d y}{r_{p}} \frac{\beta_{w}{ }^{\beta} h}{2 r_{p}+\beta_{w} R_{c}}
$$

This contributicn remains positive in $T M X(N)$ for a $11 B_{W}$ because $R_{c}>0$; in EBT it can change sign due to $R_{C}<0$. Our code which calculates interchange stability assumes a plug contribution given by

$$
\int_{p l u g} d 1 \frac{B_{t g t}}{r_{p} R_{c}} \text {. }
$$

When hot electrons are present, the code results can be used provided the total $\beta$ is replaced by an effective $\beta$-value

$$
B_{\text {tot }}+B_{\text {eff }} \equiv \frac{B_{w} B_{h}}{B_{w}+\left(2 r_{p} / R_{c}\right)}
$$

For the nominal TMX(N) operating Farameters (Section 10), we calculate a range of effective betas of $0.03<B_{\text {eff }}<0.3$ available to provide MHD stability. The lower 1 imit corresponds to $T_{e h}=20 \mathrm{keV}$ and the upper 1 imit to $\mathrm{T}_{\text {eh }}=50 \mathrm{keV}$ with $n_{i}=n_{\text {eh }}=4 \times 10^{12} \mathrm{~cm}^{-3}, T_{i}=1 \mathrm{keV}, r_{p}=10 \mathrm{~cm}$, and $R_{c}=953 \mathrm{~cm} \mathrm{also}$ used. It is noted here that $B_{\text {eff }}$ is considerably less than the hot beta range, $0.13<B_{h}<0.32$, which would be available to provide stability if the hot electrons were to behave as an MHD fluid. In the flute limit, assuming FLR stabilization of ballooning modes, the central-cell beta can be three times $B_{\text {eff }}{ }^{2}$ Therefore, we expect the maximum central-cell beta to be 1 imited by MHD to $0.09<B_{\mathrm{c}}<0.3$. 
SECTION 8

RADIAL TRANSPORT IN THE CENTRAL CELL

(S. L. Allen)

The previous sections of this report have been concerned prima iiy wich the axial confinement of the plasma and the potential profiles and plasma parameters required to achieve significant confinement times. Of equal importance is the radial confinement of the plasma which is also influericed by the plasma potentials. In particular, it is important to investigate the effects of the negative potential on radial transport and how this differs from radial transport in the positive configuration of TMX.

The so-called "classical diffusion" is present in the same form in TMX-N and TMX(P); that is, it does not depend on the plasma potential. The flux of an ion of charge $z_{i}$ due to collisions with a "background ion" of charge $z_{b}$ is given by:

$$
\Gamma_{i}(\text { classical })=n\left(n_{i} z_{i} \nabla n_{b}-n_{b} z_{b} 7 n_{i}\right)
$$

whers

$$
n \equiv \frac{4}{3}\left(\frac{2 \pi m_{b} c^{2}}{k T}\right)^{1 / 2} \frac{c e^{2}}{B^{2}} \ln \lambda
$$

with $n_{i}=$ ion density, $n_{b}=$ background density, $\ln \lambda=$ Coulomb logarithm, $B=$ magnetic field, and $m=$ background $i$ in mass. For plasma (deuterium or (uydrogen) transport, the "background" species are impuritoies. If bath the plasma and impurity radial density profilss are peaked on axis and have similar scale lengths (i.e., equal gradients), the first termi will be greater than the second by a factor of $z_{i} / Z_{b}$ and the net transport will be outward. If the impurity density profiles are peaked off axis, the situation is more complicated. In the case of impurity transport, the "background" species is the plasma; the first term is an inward fiux and the second term is the so-calied "spreading term", as it tends to broaden the radial profile (note that this is a combination of inward and outward transport terms for an impurity profile that is peaked off axis). 
Added to the classical transport is the so-called neoclassical transpart. In many cases, this transport can be significantly larger than classical diffusion. Neoclassical diffusion is caused by the quadrupole component of the non-axisymmetric magnetic field in the transition regions between the central cell and the end cells. These quadrupole fields cause a radial displacement which is proportional to $\cos 2 \psi$, where $\psi$ is the azimuthal rotation angle. In the TMX device, one end cell is rotated by $90^{\circ}$ with respect to the other and this radial disphacenent is cancelled [except for a residual cos $(4 \psi)$ component]. However, if the particle drifts azimuthally as it transits from one end to the other, these radial displacements do not cancel, and radiai transport results. One cause for such an azimuthal drift is an EXB drift caused by a radial electric field in the central cel1. Three classes of transport have been identified based on the parameter $\Delta \psi$, the ratio of the drift frequency $\nu_{d}$ to the bounce frequency $v_{b}$ (the bounce frequency is the reciprocal of the transit time between the end plugs):
1. $\Delta \psi \ll 1$
neoclassical transport
2. $\Delta \psi>1$
is
resonant neoclassical transport
3. $\Delta \psi \gg 1$
stochastic diffusion.

In both TMX configurations, resonant neoclassical transport is the dominant mechanism. The term "resonant" means that $\Delta \psi$ is on the order of ore and an ior drifts about an additional $90^{\circ}$ in azimuth per transit. This cancels the $90^{\circ}$ difference between the end cells and resuits in radial transport due to non-cancellation of the radial displacements. The detailed theory of resonant neoclassical transport has been derived by $R$. Cohen, et al. and is described in Refs. 1 and 2. It was found that the diffusion caefficient for resonant neoclassical transport depends on $v_{c} / v_{b}$ where $v_{c}$ is the collision frequency (see Fig. 8-1). In the collisional regime $\left.\left\langle u_{c} / \nu_{b}\right\rangle 1\right)$ the diffusion coefficient also depends on whether the ratio $v_{d} / v_{b}$ is greater or less than one (see Fig. 8-1). The background plasma is primarily in the intermediate or plateau regime, while the impurities are in the collisional regime. However, for the light impurities in a $T M X-(P)$ plasma, $v_{d} / v_{b} \gg 1$ (the solid line in Fig. 8-1) and $1 n\left(v_{c} / v_{b}\right)$ is greater than $\ln (1)$ but less than $\ln \left(v_{d} / v_{b}\right)$. As shown in Fig. $8-1$, this means 


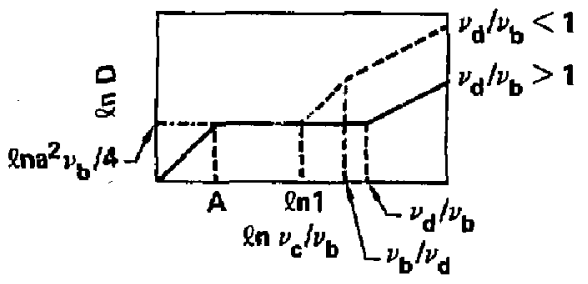

Figure 8-1. Diffusion coefficient vs collisionality paraneter. 
that the diffusion efficients for the background plasma and the impurities have the same form, $i . e . D$ is constant with $v_{c} / v_{b}$. The flux is given by:

$$
\underset{\text { neoclassical })}{\Gamma(\text { resonant }}=D_{0}\left(-\nabla n_{i}-\frac{n_{i} z_{i}}{T_{i}} \nabla_{\phi}\right)-\frac{3}{2} D_{0} \frac{n_{i}}{T_{i}} \nabla_{i},
$$

where

$$
D_{0}=1310 T_{i}^{3 / 2} r^{2} z^{-2}\left(\frac{x^{6}}{1+x^{6}}\right) \text { (oxygen) }
$$

and

$$
X=1828 T^{-1 / 2}\left(\frac{-1}{r} \frac{\partial \phi}{\partial T}\right) .
$$

Recall that this is for impurities in the regime

$$
\Delta \phi \approx \frac{v_{d}}{v_{b}} \gg 1 \text { and } \frac{v_{d}}{v_{c}} \gg 1
$$

for the parameters $B_{c}=1000 \mathrm{G}, m_{i}=16, m_{b}=2, L_{c}=315 \mathrm{~cm}$, and $L_{t r}=$ $100 \mathrm{~cm}$ ( $L_{c}$ is the length of the central cell and $L_{t r}$ is the length of the transition region between the solenoidal central-cell field and the minimum- $B$ end-cell field). The parameters given are for oxygen; the coefficients for a deuterium or nydrogen background are different, but the form is the same.

Note carefully the dependence of the flux on the radial potential profile. In the case of $T M X(P)$, the potential profile should be similar to that of TMX. That is, peaked on axis so that $\nabla_{\varphi}$ is negative and the resulting fiux due to resonant neoclassical transport is outward. For peaked plasma profiles, $\nabla n_{i}$ is also negative so the net flux is outward [see Eq. (2)]. However, in TMX(N), the radial potential may be negative with respect to the wall so $\nabla \varphi$ will be positive and the flux wil * - inward due to this term. For deuterium, $z_{i}=1$, the flux [ $N$ " corresponds to TMX(N) and "P" corresponds to $\operatorname{TMX}(P)]$,

$$
\Gamma\left(\begin{array}{l}
N \\
p
\end{array}\right)=-\nabla n_{i}+\frac{n_{i}}{T_{i}}|\nabla \psi|-\frac{3}{2} \frac{n_{i}}{T_{i}} \nabla T_{i}=\frac{n_{i}}{r_{n s}}+\frac{n_{i}}{T_{i}} \frac{\varphi_{i}}{r_{p s}}-\frac{3}{2} \frac{n_{i}}{r_{t s}},
$$


where $r_{n s}$ is the density scale length, $r_{p s}$ is the potential scale length, and $r_{t s}$ is the temperature scale length. If we assume that these are equal, then

$$
\Gamma\left(\begin{array}{l}
N \\
p
\end{array}\right)=\frac{n}{r}\left(+1 \mp \frac{\varphi_{i}}{T_{i}} \mp \frac{3}{2}\right)=\frac{n}{r}\left(-\frac{1}{2} \mp \frac{\varphi_{i}}{T_{i}}\right) .
$$

Hence for $T M X(P)$, as long as $\varphi_{i} / T_{i}>1 / 2$, the transport will be outward. The projected parameters for TMK(P) (Section 10) indicate $\varphi_{j} / T_{j}$ $\sim 2.2 / 0.9 \sim 2.5$ so the net outward flux will be $+2.5 \mathrm{n} / \mathrm{r}$ in this case. However, in the case of $\operatorname{TMX}(N)$, with $\varphi_{j} / T_{i}=2.5 / 1$. , the net flux will be $-2.0 \mathrm{n} / \mathrm{r}$ inward. Therefore, the radial transport may be very different in $\operatorname{TMX}(\mathrm{N})$.

The impurity radial transport may also be very different for $\operatorname{TMX}(N)$ as colpared with TMX(P). Fig. 8-2 shows the radial flux of OIII for the case of the previous (positive) TMX experiment. Note that in the case of impurity transport the classical and resonant neoclassical fluxes are ahout the same magnitude, however, the classical term is inward whereas the resonant neoclassical contribution is outward. This plot is for the case af peaked impurity and background-plasma profiles. In a negative configuration the resonant neoclassical term would be more negative than the classical term and thus the total would be inward. A similar result is obtained when the impurity profile is peaked off axjs, as shown in Fig. 8-3. Note that the classica? term changes sign and that the neoclassical term is dominated by the potential term. The fact that the flux is strictly inward could be harmful; however, the experimental results from the earlier positive TMX experiment indicate that an additional outward term may be present. As yet, this is not completely understood, but indicates that the net flux even in $\operatorname{TMX}(N)$ may be outward.

In summary, the presence of a negative potential in $T M X(N)$ will change the sign on the mobility term for resonant neoclassical transport. The deuterium transport may be inward and improves radial confinement. The resonant neoclassical theory predicts inward impurity transport, though other more important mechanisms may cause outward radial transport. 


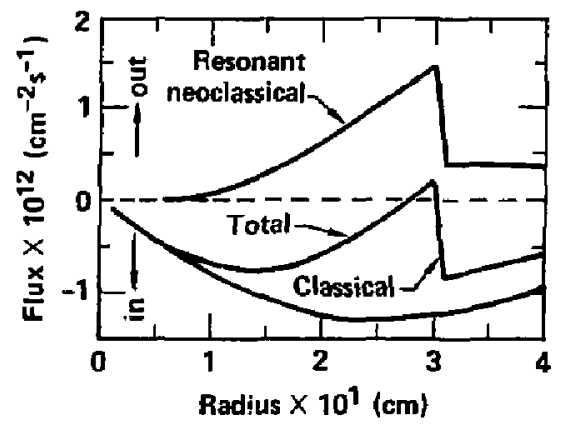

Figure 8-2. Theoretical fluxes - 0 III, gas box operation. 


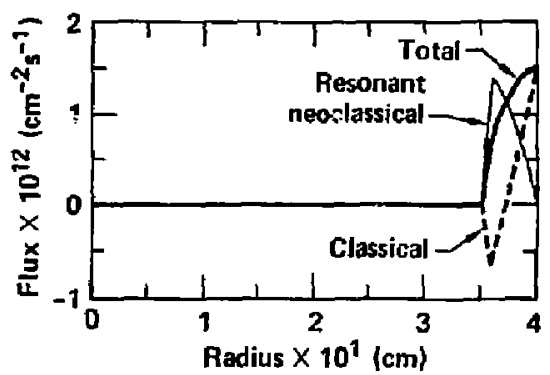

Figure 8-3. Theoretical fluxes - 0 II!, puffer operation - shell ion iensity model.

$-99-$ 
In this section we describe a start-up scenario (Fig. 9-1) for the negative aperation of TMX. In this scenario, plasma is initially injected axially by the washer-stack guns on each end of the device. These guns remain on until the central-cell density $\left(n_{c}\right)$ is of order $2 \times 10^{11} \mathrm{~cm}^{-3}$ and the end-cell density $\left(n_{p}\right)$ is of order $5 \times 10^{11} \mathrm{~cm}^{-3}$. At this time the plasma potentiai is positive with respect to the vacuum container walls (ground potential).

When the specified densities are reached,* the plasma guns are turned off ("crow-barred" off), thus breaking a strang electron-thermal-conduction path to the end walls and the ECRH at both $l \omega_{c e}$ and $2 \omega_{c e}$ in the eno cells is turned on. In addition, gas is introduced into the central cell to maintain the plasma. During this phase of the start-up, hot electron plasmas are formed in the end cells to provide an MHD anchor for the system. Since the anchoring depends upon the hot electron beta $\left[\beta_{\text {eh }}=n_{e h} T_{e h} /\left(B^{2} / 8 \pi\right)\right]$ we choose to achieve stability with high electron temperature. We keep the hot electron density low $\left(-5 \times 10^{11}\right)$ and allow their energy to increase to the limit set by the resonant frequency shift caused by the relativistic mass change $\left(T_{\text {eh }} \approx 20 \mathrm{keV}\right.$ to $\left.50 \mathrm{keV}\right)$. By doing so, we minimize the scattering lass current of the hot electrons ( $I_{\text {scatt }} \alpha n_{h}^{2} / T_{\text {eh }}^{3 / 2}$ ) while still establishing a hot-electron anchor. The plasma density and potential profiles for this stage of start-up are shown in Fig. 9-2. Figures 9-3 and 9-4 present schematic diagrams of the corresponding particla and power flows.

At this point the system is MHD stable, so we begin to sequence on the central-cel1 neutral beams to accomplish two very important effects: (1) Drag

\footnotetext{
* Since the electron energy confinement time is less than the particle confinement $t$ ime it is important to crow-bar the guns off when the desired densities are first reached, as opposed to letting the density overshoot and waiting for it to decay back to the desired values. 0therwise, $\mathrm{T}_{\mathrm{ec}}$ will fall too low to efficientily source the hot electrons.
} 


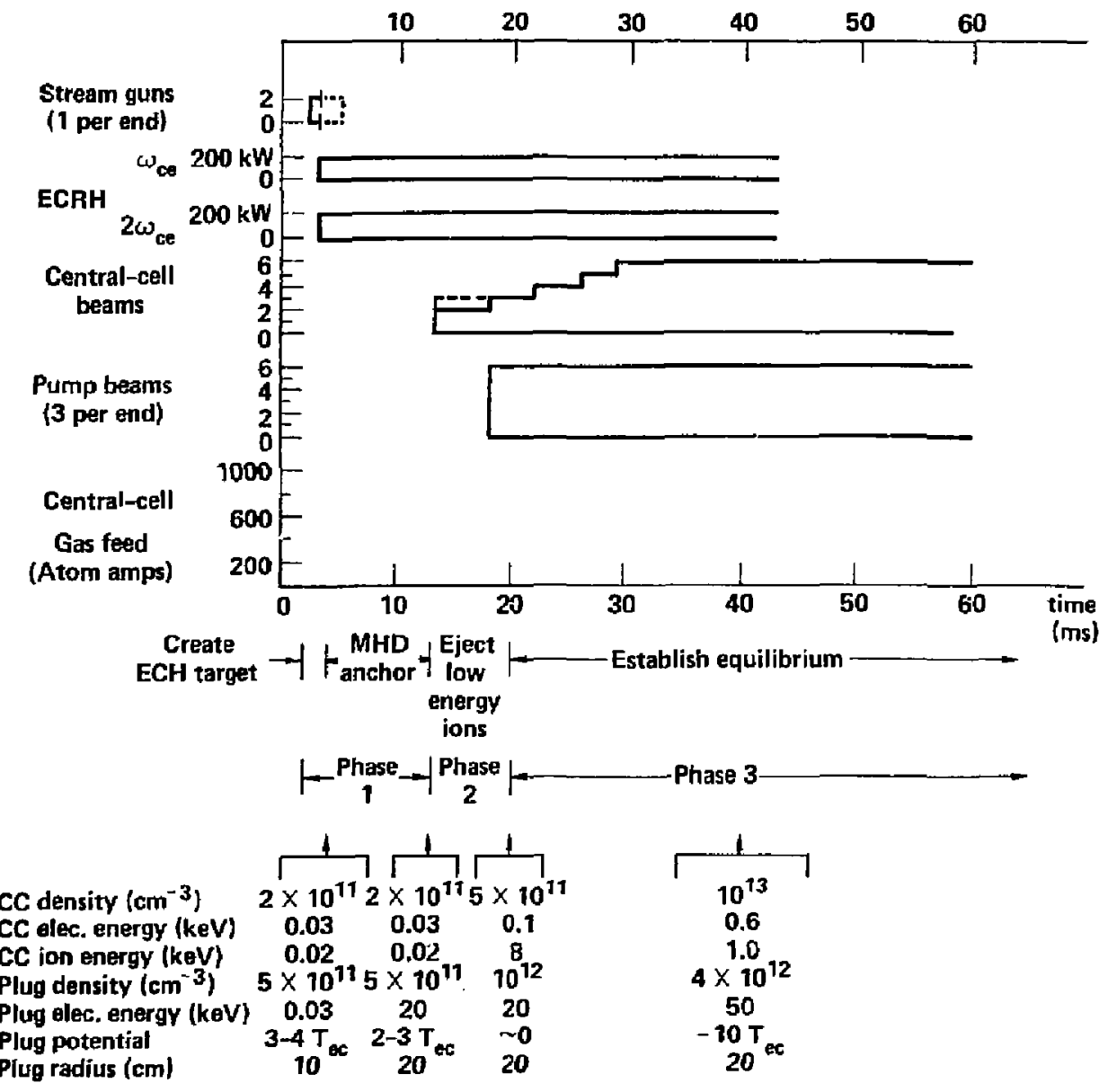

Figure 9-1. Start-up sequence. 


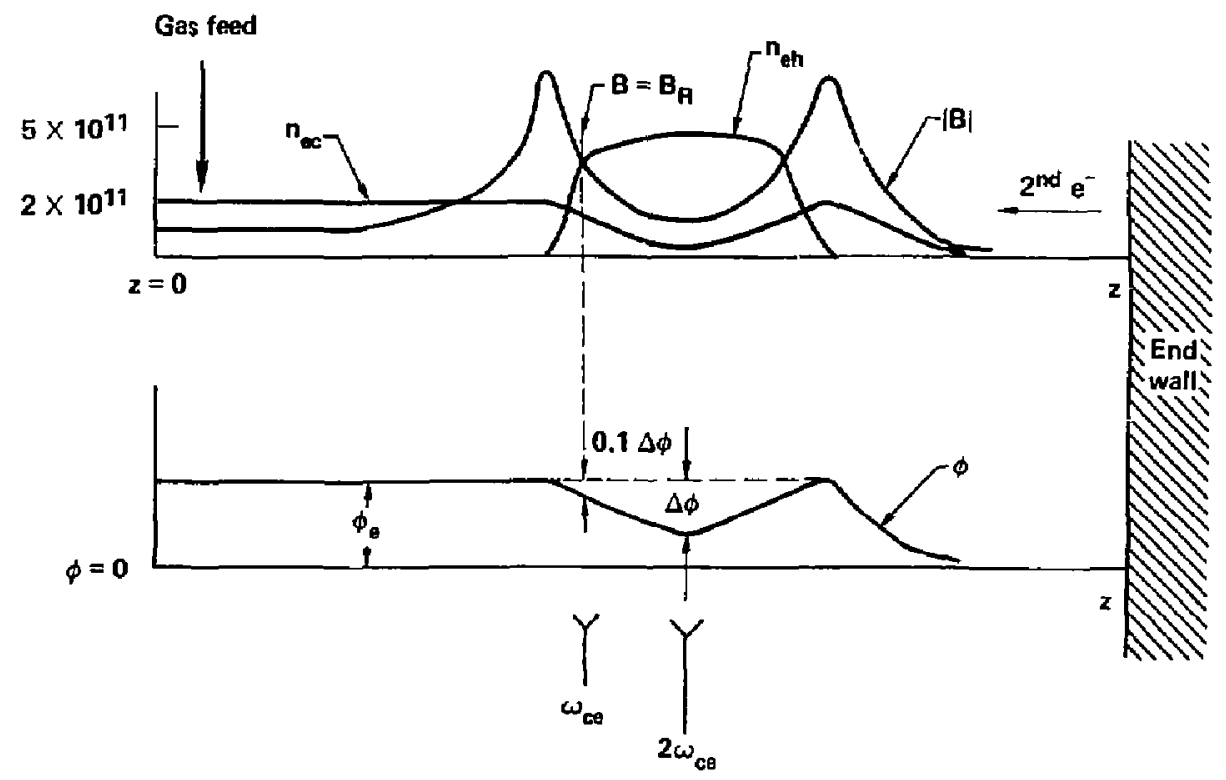

Figure 9-2. Phase 1 axial profiles. 


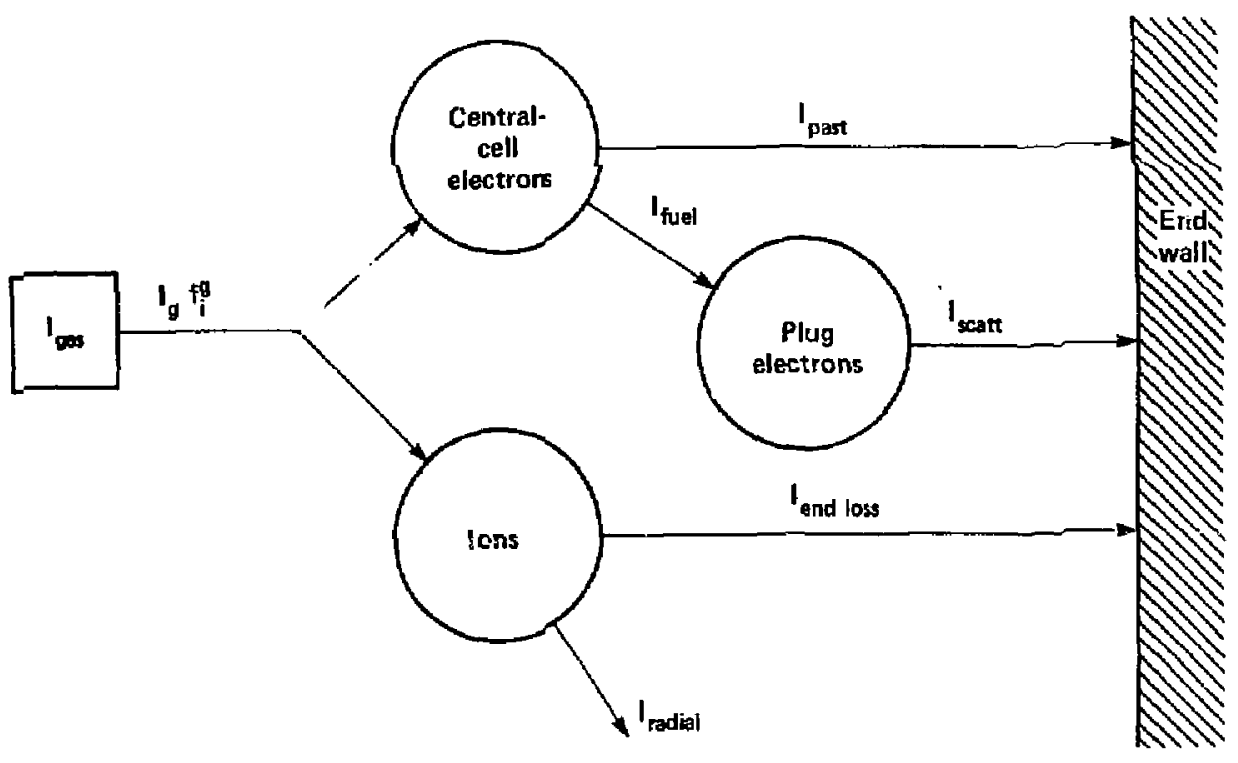

Figure 9-3. Phase 1 particle flow. 


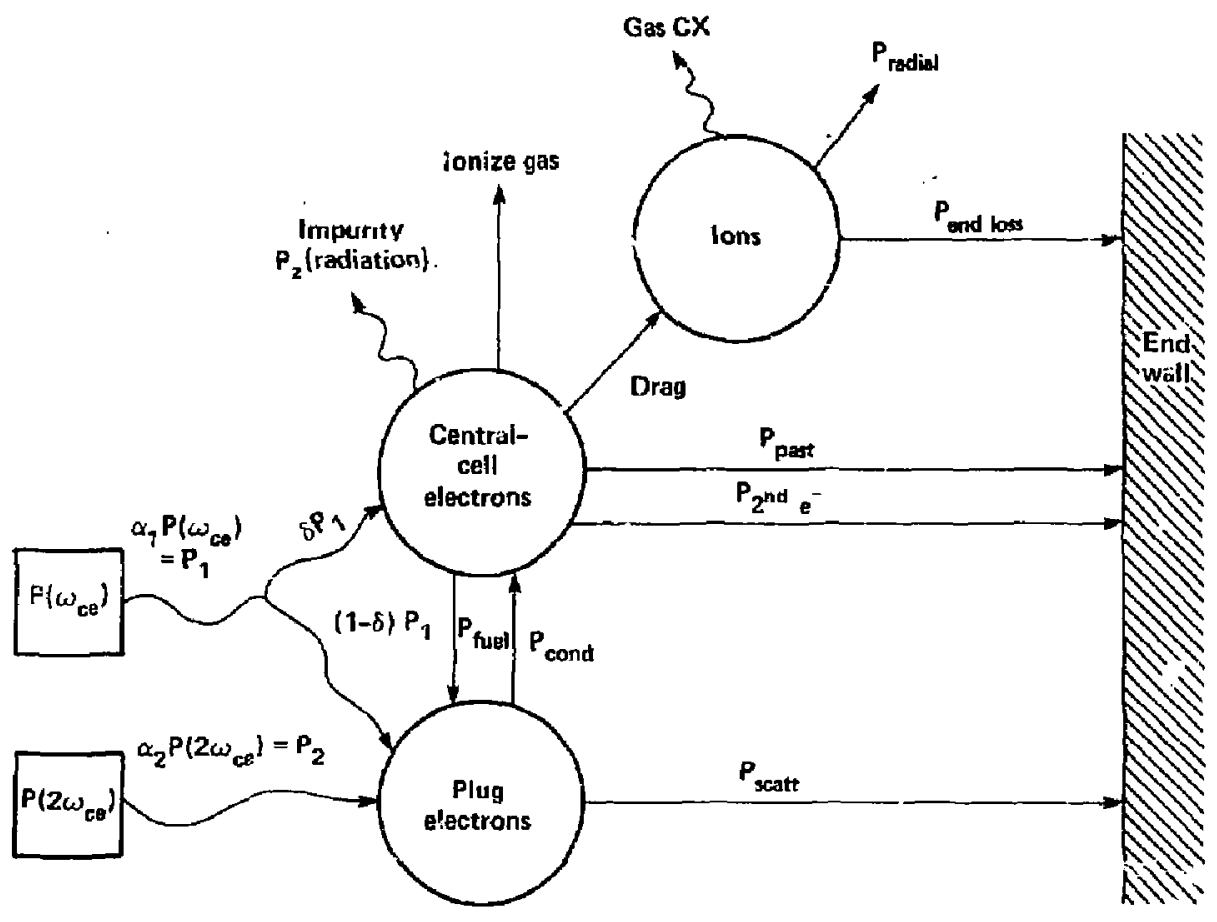

Figure 9-4. Phase 1 power flow. 
on the hot ions raises the central-cell electron temperature so that the build-up rate of the hot electrons in the end cells is increased and (2) A potentid drop between the midplane of the central cell and the inner mirror develops due ta the mirror-confined ions in the central cell. This positive ambipolar potential drop reduces the confinement of the low energy central-cell ions so that the barrier filling rate is significantly reduced. After the turn on of the central-cell beams, therefore, the pump beams are sequenced on and the potential of the entire plasma can now be driven-negative.

At this point the rest of the central-cell beams are sequenced on and the gas feed to the central cell is increased to fill the central cell to the final, desired density $\left(\approx 10^{13} \mathrm{~cm}^{-3}\right)$. However, the turning on of the additional beams and gas is constrained by the continuing build-up of tile hot end-cell electrons so that MHO stability is preserved. The total time required for build-up to the final steady-state is estimated to be 30 to $90 \mathrm{~ms}$.

In the rest of this section we detait the refevant equations and logic involved in the derivation of this scenario.

\subsection{Prase T - Establishing ail MHD Anchor}

The purpose of the first phase of the start-up is to provide a suitable initial plasma to serve as a source for electron-cyciotron-resonance heating (ECRH) so that we can establish an MHD anchor for the sysiem. Since the maximum experimental duration is $75 \mathrm{~ms}$, it is essential triat we get through this phase of start-up as quickly as possible.

Ta do this, we keep the plasma density low so that the absorption of the fundamerital ECRH power is maximized [Eq. (AT.5), all equations appoar in the appendix] and the scattering losses of the hot electroris are minimized $[\mathrm{Eq} .(A 1.6)]$. Under these conditions we expect the hot electron temperature to increase rapidly to a maximum vaTue which is Timited by the retativistio mass shift of the electrons. ' The expected temperatures correspond to an eler tron energy in the range of $20 \mathrm{keV}$ to $50 \mathrm{kcV}$. 
9.9.1. Plasma Initiation. The first step in the start-up procedure is to provide a suitable fuel plasma for ECRH. We require that this plasma have relatively low density, $\mathrm{n} \sim 2 \times 10^{11} \mathrm{~cm}^{-3}$, with a warm electron temperature of at least $T_{e} \geq 30 \mathrm{eV}$, so that fundamental $\left(\omega=\omega_{\mathrm{ce}}\right.$ ) ECRH effectively traps and heats the central-cell electrons [Eqs. (AT.2b) and (Al.5)].

We propose to use streaming plasma guns to produce the initial plasma for ECRH. Since we do not require that these guns also provide a target plasina for neutral beams, the negative operation of TMX is less demanding on the type and location of the plasma guns than is the positive machine. Any location which fills the machine with a plasma of radius 10 to $15 \mathrm{~cm}$ with the stated density and electron temperature in a reasonable amount of time ( 1 to 5 ms) will be acceptable.

The present mounting of the MFTF style guns ${ }^{2}$ meets these requirements. Furthermore, measurements of the gun plasma by 0 sher ${ }^{3}$ show that this type of gun is capable of producing tise desired plasmas. For the purpose of this study, therefore, we will assume that these guns will te used for the start-up of the negative machine.

The time required to $\mathrm{fill}$ an end cell by trapping of the stream-gun plasma using two of these guns (one per end) can be estimaias using the desired volume and density of the plasma and the output current of the gun,

$$
\Delta \mathrm{t} \simeq \frac{n V e}{\alpha_{p} l}=\frac{\left(5 \times 10^{11}\right) 6 \times 10^{4}\left(1.6 \times 10^{-19}\right)}{\alpha_{p} 500}=\frac{9 . \mu \mathrm{s}}{\alpha_{p}},
$$

where we have assumed a plasma radius of $r_{p} \approx 10 \mathrm{~cm}$ from the present stream-gun flux-tube mapping with $L_{\text {eff }}^{p}=213 \mathrm{~cm}$ the plasma length. In the above equation, $a_{p}$ is the fraction of the piasma current which is stopped in the plug. For a choice of $\alpha_{p} \simeq 0.01$ the required build-up time is $900 \mu 5$. In this same time the central cell would fill up to

$$
n_{c}=\left(\frac{r_{p}}{r_{r}}\right)^{2}\left(\frac{L_{\text {eff }}^{p}}{L_{e f f}^{c}}\right) n_{p}=\left(\frac{3}{5}\right)\left(\frac{213}{508}\right) n_{p}=\frac{1}{4} n_{p}
$$


if the stopping fraction is the same in the central cell and the plug, j.e., $\alpha_{c}=a_{p}$, and $r_{c}, L_{e f f}^{c}$ are the central-cell plasma radius and 1ength. Since the central cell is longer with a larger mirror ratio, we might expect that $\alpha_{c}>\alpha_{p}$ due to an increased probability of scattering into the trapped region of velocity space. Therefore, within the uncertainties in our model, we expect

$$
n_{c}=\frac{n_{p}}{4} \text { to } n_{p}
$$

9.1.2. ECRH Turn-On. When the desired source plasma has been formed, the ECRH gyrotrons are turned on. The absorption of the $2 \omega_{c e}$ power will initially be very low due to the low electron temperature [Eq. (A1.4)]. However, the fundamental heating should soon $(0.05 \mathrm{~ms})$ raise the plug electron temperature to a 100 to $200 \mathrm{eV}$ where the absorption of the second harmonic power is 10 to $20 \%$ efficient at $n \simeq 5 \times 10^{11}$. Therefore, we propose to turn on both the fundamental and second harmonic heating gyrotrons at the same time.

Ouring this phase of the start-up, neutral gas will be injected into the central cell with a programmed gas feed to replace the plasma losses due to both Pastukhov losses of the central-cell electrons and scattering losses of the plug eiectrons.

Figure $9-4$ is a schematic representation of the power flow dur in this phase of start-up. The ECRH is the only source of power to the system at this time. This power is absorbed, predominantly by the mirror-confined plug electrons. Some power, however, is absorbed by the central-cei i slectrons which pass through the fundamental resonance. This power is carried into the central cell where it is shared with the bulk of the central-cell electrons by drag and trapping of the passing electrons (by pitch-angle scattering).

The piug e iectrons lose most of their power due to pitch-ang iescattering particle loss. At equilibrium, almost all of the rest of their power is lost by thermal conduction to the central-cell electrons, anly a very small amount of power is transferred to the central-cell ions by drag on the hot electrons. 
The centra1-cell electrons gain energy by ECRH heating of the passing electrons and by drag on the hot electrons. They in turn lose energy by axial particle escape (Pastukhov losses), secondary electron emission from the end walls (the plasma potential is still positive), ionization of the fueling gas, and drag by the central-cell ions.

Finally, the central cell ions lose their power by axial particle escape and charge exchange with neutral gas (predominantly from fueling).

It seems unlikely that it will be desirable to allow this phase of the start-up to evolve to steady-state. We anticipate that the time-dependent start-up code now under development will show that we can begin the next phase of build-up without ever reaching the equilibrium of the first phase.

\subsection{PHASE 2 - TURNING ON THE CENTRAL-CELL NEUTRAL BEAMS}

After we have established an MHD anchor for the system, the next step in the build-up is to modify the existing central-cell plasma so that the available puming-beam current is sufficient to allow us to drive the plasma potential strongly negative by pumping ions out of the plugs. To accomplish this, we now sequence on the central-cell neutral beams.

At this point two important chat: res in the central-cell plasma take place. Firstly, the mirror-confined hot ions deposited in the plasma strongly heat the central-cell electrons by drag. This increases the fueling rate of the hot electrons [Eq. (AT-2b)] so that the hot electron build-up rate increases. Secondly, the hot, mirror-confined central-cell ions (fram beam injection) change the axial potential profile so that cold central-cell ions are not confined.

Up until the time that we turned on the central-cell beams, TMX was operating, essentially, in a kelly mode, ${ }^{4}$ i.e., there was no potential drop between the midplane of the centrat cell and the inboard mirror of the plug, Fig. 9-1. However, the mirror-confined ions from the beams cause a potential drop to form of order $T_{e c} \ln [n(z=0) / n$ (mirror)]. Since this is several times the cold ion temperature calculated for phase $1\left(T_{i c} \approx 10 \mathrm{eV}\right)$ most of the coid ions will be ejected from the central cell and lost to the end wall as the plasma potential builds up (Fig. 9-5). 


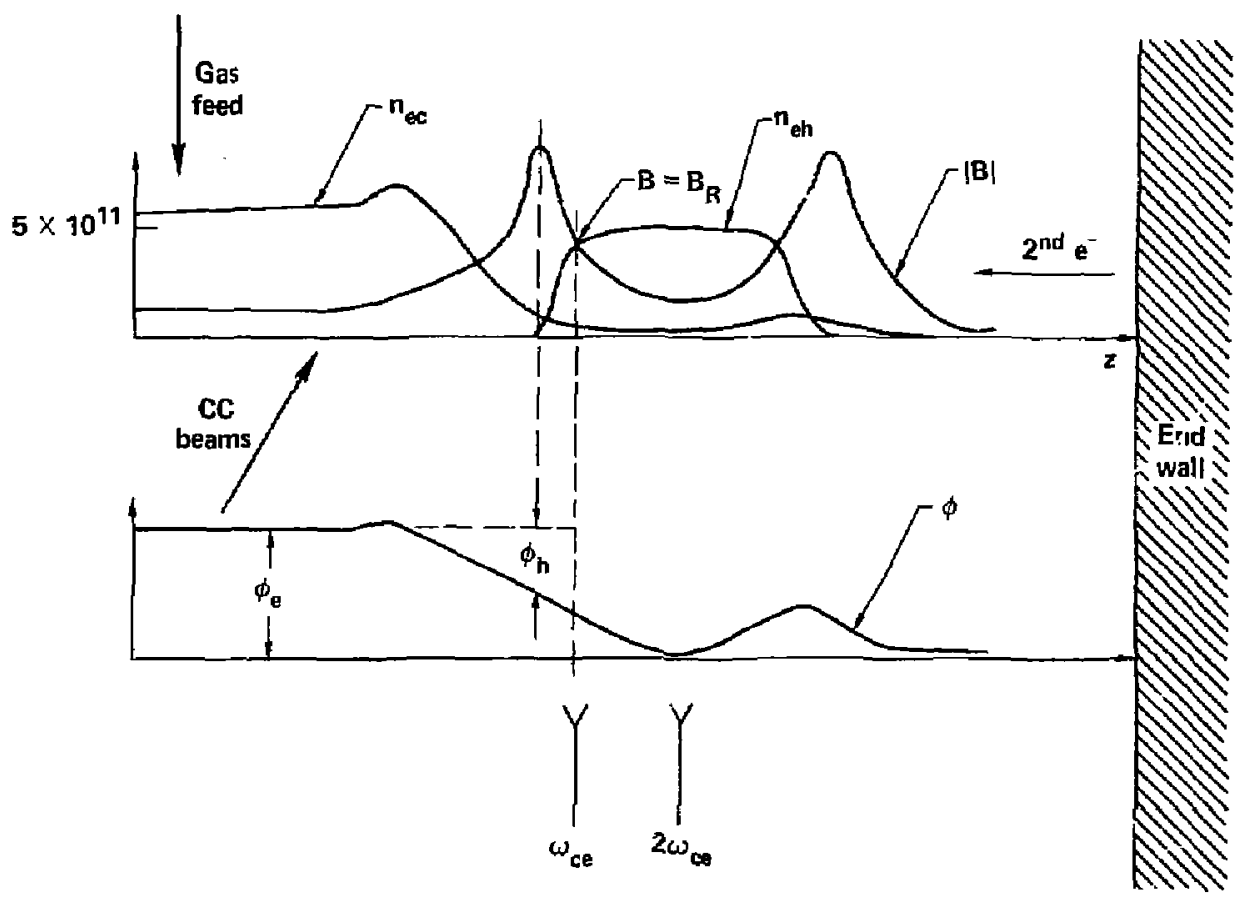

Figure 9-5. Phase 2 axial profiles. 
This ejection of the cold ions from the device is highly desirable because it significantly reduces the filling rate ${ }^{5}$ of potential minimum in the plug which is proportional to $1 / T_{i}^{3 / 2}$. At this point, therefore, the available pumping current $(\sim 120 \mathrm{~A} / \mathrm{plug})$ can pump out the plug potential minimum and drive the plasma prtential negative (see phase 3 ).

The proper sequencing on of the central-cell neutral beams at this time is the most critical aspect of this phase of start-up. In order to maintain central-cell stability--both microstability and MHD stability--we do not wish to over fill the central cell with hot ions.

The first stability issue, that of microstability, arises due to the loss cone nature of the central-cell ion distribution once the cold ions are ejected. This, however, does not appear to be a major constraint because the large central-cell mirror ratio and the low ion density leads to a small quasilinear stream current requirement [Eq. (A3.12)]: nominally, the calculated stream current requirements are less than the calculated trapped beam current, $I_{b} f_{j}$. In addition, the beam injected ions $\left(60^{\circ}\right.$ injection angle) form a s Toshing ion distribution which tends to be more stable to the OCLC instability 50 the stream requirements should be easity met.

The more serious constraint on the central-cell hot ion density is imposed by MHD stability. During phase 1 of start-up, we established a hotelectron anchor in the good curvature of the plugs. However, the effectiveness of this anchor is strongly coupled to the centrai-cell warm plasma beta that leans into the plug region [6]. In effect, the hot piug electruns (due to their finite beta) dig a radial magnetic well that is significantly deeper than that of the vacuum field. The warm plasma particles, both ions and electrons, which are in this well then provide the stabilizing contribution to the pressure wefighted $S \mathrm{dl} / 8$.

Mathematically, this relationship is expressed by*

$$
B_{\text {eff }}=\frac{\Lambda}{1+\Lambda} B_{p},
$$

where $\beta_{\text {eff }}$ is the effective plug beta for anchoring the system, $\beta_{p}$ is the

*We have assumed here that the mode frequency, $\omega$, is greater than either the curvature, or $\nabla B$ drift frequency of the warm plasma species and js less than the $\nabla B$ or curvature drift frequencies of the hot plasma species. 6 
total plug-beta $\left(B_{p}=B_{h}\right.$, where $B_{h}$ is the hot-electron beta) and $\Lambda$ is given by

$$
\Lambda \approx \frac{1}{2} B_{w}\left(1+\beta_{p}\right) /\left(1-\beta_{p}\right) R_{c} /\left|r_{p}\right|,
$$

where $\beta_{w}$ is the warm plasma beta (both ion and electron) in the plug, $R_{C}$ is the vacuum field radius of curvature and $r_{p}$ is the plasma radius. In the limit, therefore, that the warm plasma beta goes to zero, $\beta_{\text {eff }}+0$ and the system is probabiy MHD unstable.

In our analysis of the start-up, we have set

$$
\beta_{p}=\beta_{e h}=n_{e h} k T_{e h} /\left(B^{2} / 8 \pi\right)
$$

and

$$
\left.n_{w}=\left[n_{i p}+n_{i t}\right) k T_{i c}+n_{e p} k T_{e c}\right] /\left(B^{2} / 8 \pi\right),
$$

where the sibscripts $h$ stands for hot and $p$ and $t$ refer to passing and trapped particles, respectively. We have assumed that both the trapped and passing ions have the centra] cell ion temperature, $k T_{i c}$ and the passing electrons have the central cell electron temperature, $k T_{\text {ec. The destabilizing }}$ contributions to this integral come from the central-cell plasma pressure, especially in the regions of bad curvature in the transition region. Previous stability calculations over-estimated this contribution by assuming an isotropic central-cell axial pressure profile.

Fokker-Planck code calculations ${ }^{7}$ of the TMX equilibrium with centralcell neutral beam injection show that the axial pressure profile is strongly peaked at the magnetic field which corresponds to the mirror point for ions normally injected at $60^{\circ}$ (TMX central-cell beams inject at angles of $58.5^{\circ}$ and $70^{\circ}$ from the magnetic axis). This peaxing occurs because the ions first slow down in energy at a constant pitch-angle due to electron orag and then scatter in pitch-angle due to ion-ion collisions. For this reason, the pressure profile peaks at a B-field which iies inside of the region of bad curvature in the transition region (Fig. 9-6). Based on this picture, we expect to calculate higher allowed values of central cell $B, B_{c}$, in TMX than those previously predicted using the isotropic pressure profile model. 


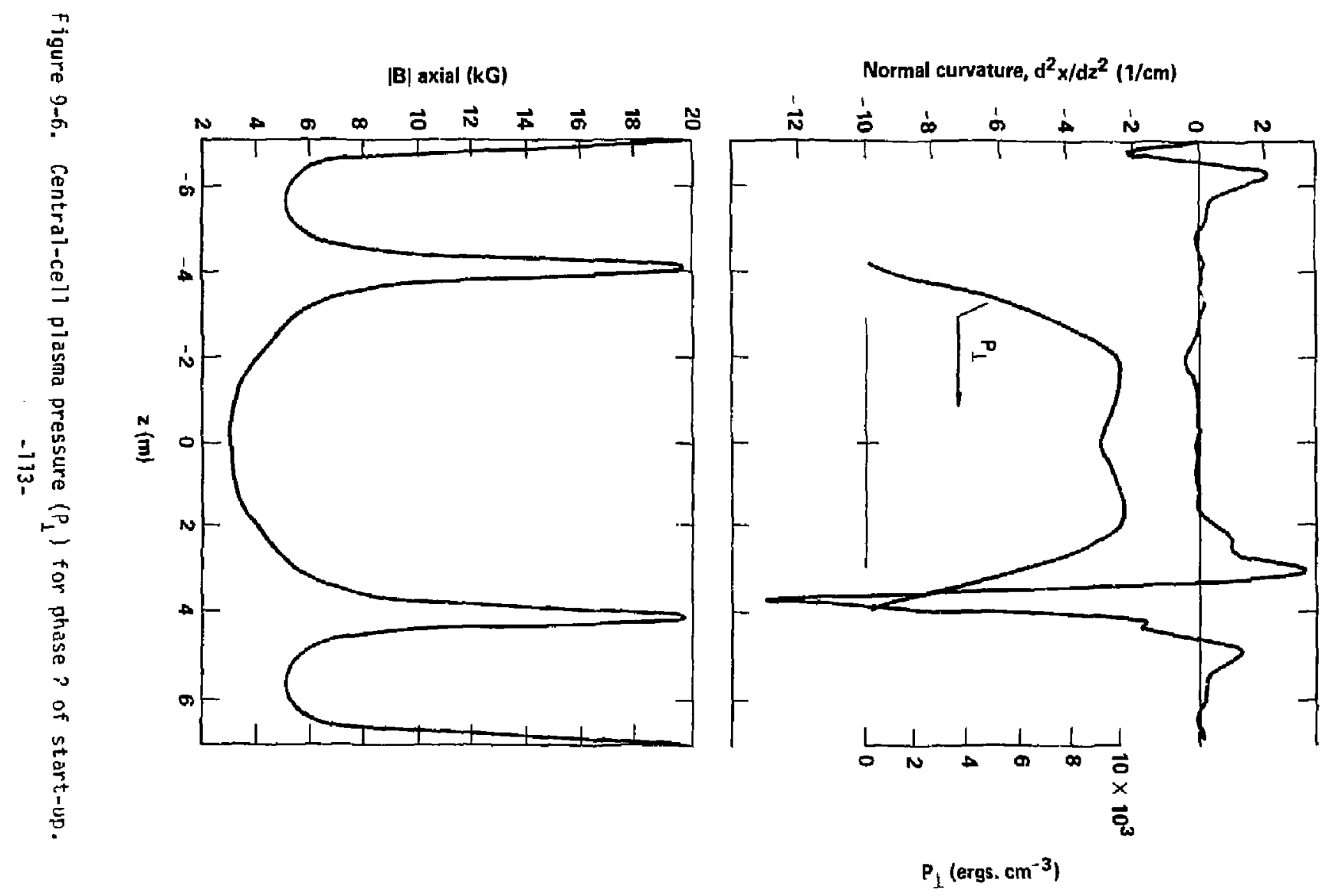


At this time $T$. Kaiser is modifying the Tebasco code ${ }^{8}$ so that it will calculate the marginal stability when the central-cell pressure is non-isotropic as it is during central-cell neutral beam heating. When the Tebasco code resu?ts are available they will be combined with the MHD analysis developed by Baldwin ${ }^{6}$ to create a more accurate picture of the beta 1 imit: in the negative mode of TMX operation. We will then be able to mora accurately predict the turn-on sequence of the central-cell neutral beams which will allow us to bring up both the central-cell hot-ion density and the plug hot-electron density together so that MHD stability is maintained. For the purpose of this report, we have mede the pessimistic assumption that the previously calculated ${ }^{9}$ ratio of $B_{c} / B_{p} \sim 1$ still holds.

As was the case in the previous phase of start-up, it is unljkely that we will need to wait until the plasma reaches equilibrium before we begin the next phase.

\subsection{FINAL PHASES - PUMP BEAM TURN-ON AND EVOLUTION TO EQUILIBRIUM.}

Soon after the central-cell beams were turned on during phase 2, the cold centrai-cel] ions were ejected from the machine by the plasma potentia]. At this time, the pump beams are turned on and the ions are pumped out of the minimum in the plug potential. This drives the end-cell poten'ial strongly negative and both increases the confinement of the central-cell electrons and stops the flow of the secondary electrons from the end wall (Fig. 9-7).

The first effect (increased central-cell electron confi rement) causes the central-cell potential to go negative to equalize the roni inement of ions and electrons by establishing electrostat ic confinement of the central-cell ions. The second effect (stopping the end-wall secondary electrons) reduces the electron power loss so $T_{\text {ec }}$ increases and, therefore, so does the fueling rate of the hot electrons.

At this point additional gas is introduced into the central cell to fuel the now confined low-energy central-cell ions and the remaining centralcell and punip beans can be sequenced on to complete the evalution to equilibrium (see Sec. 10). This completes the final step in start-up. 


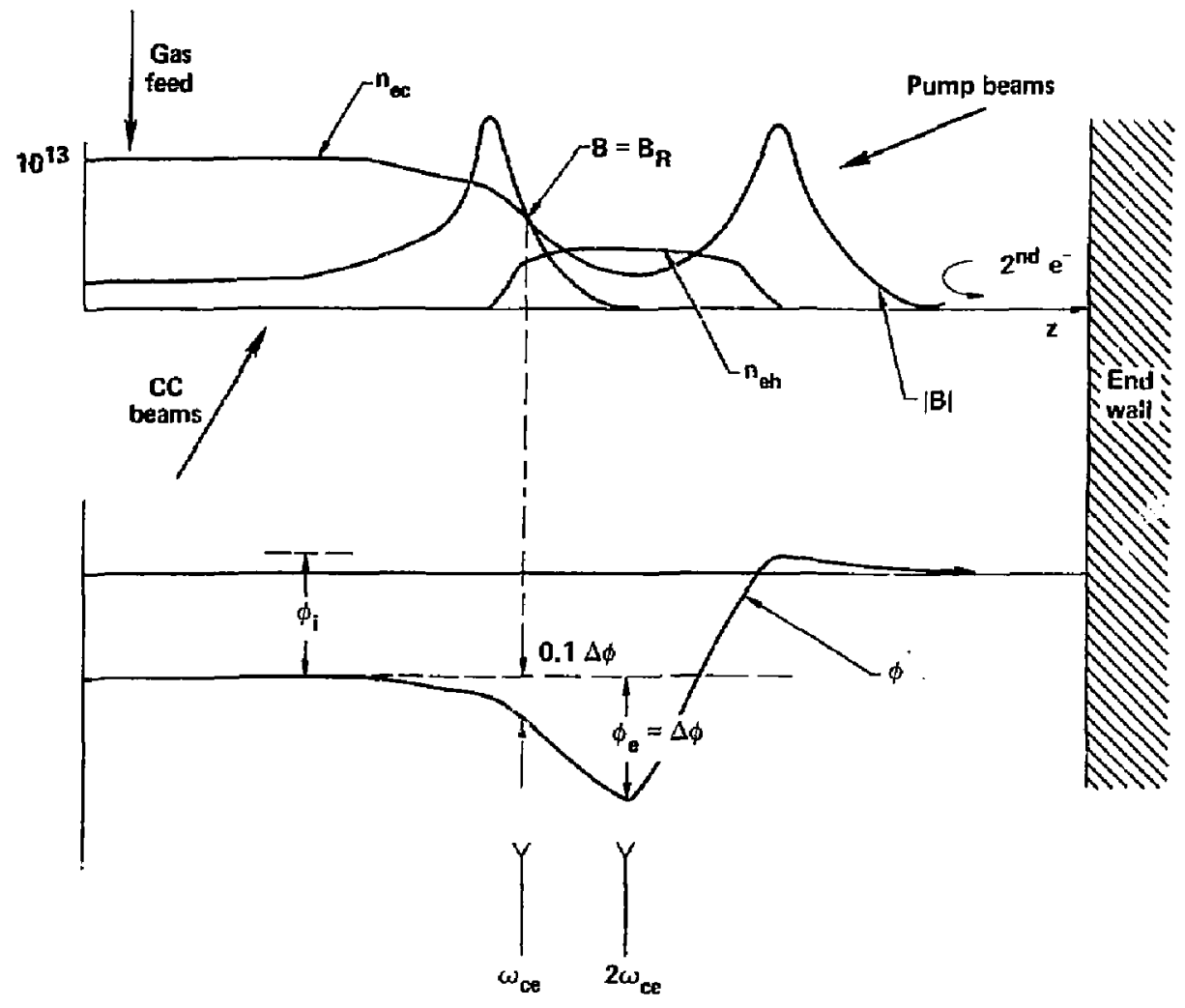

Figure 9-7. Phase 3 axial profiles. 
Throughout this start-up sequence, we have minimized the time required to reach the desired plasma states. The build-up code presently under development by the authors of this section will be used to further define the time history of the plasma during start-up to insure that the desired steady state can be reached during the allowed time. 


\section{SECTION 10}

DPERATING PARAMETERS

\section{(W. E. Nexsen)}

The particle and power balance equations are given in Appendix $B$. To compare the negative mode of operation with the positive mode outlinad in the TMX-U proposal, we have found the steady-state solutions of these equations using the proposal values for the central-cell Pastukhov confinement parameter $(n \tau)_{\| 1}=10^{12} \mathrm{~cm}^{-3} \cdot \mathrm{s}$ and a plug density at the electron feed point of $7 \times 10^{12} \mathrm{~cm}^{-3}$. The mirror-confined hot electron population in the plug will be fed from the central-cell electron population. Since these electrons do not extend past the negative potential minimum which, to minimize sheath fomation, must be located at the plug magnetic field minimum, the feed mist be located on the central-cell side of the minimum and at the $10 \mathrm{kilogauss}$ resonant field value (for resonance at the $28-\mathrm{GHz}$ gyrotron frecuency).

To avoid sheath formation in the region between the inser mirror peak and the field minimum, it is necessary that the density drop monotonically with decreasing magnetic field and that enough ions be trapped in the potentia] well so that

$$
g=\frac{n_{i t}+n_{i p}}{n_{i p}}=6 .
$$

We model the density behavior in the regian between the inner mirrar and the field mirror by setting

$$
n(B)=m_{m j}\left(\frac{B}{B_{m i}}\right)^{k},
$$

where for $\operatorname{TMX}(N)$ parameters, $k \approx 0.6$ yields $g \approx 6$. Setting the density at the $10-k G$ feedpoint to $7 \times 10^{12}$, equation (1) with $k=0.6$ yields a value for $n_{m i}$, the density at the inner mirror peak $\left(B_{m i}=20 k G\right)$

$$
n_{m i}=7 \times 10^{12}(2)^{0.6}=1.06 \times 10^{13} .
$$


For the purpose of comparison with the positive case we will assume that $n_{m i}=n_{c}$, the density on axis at the central-cell midplane of the potential-confined ions. In doing this, we ignore the axial potential variation in the central cell due to the non-Maxwellian mirror-contained hot ion component.

A. Pastukhov confinement parameter $(n \tau)_{11}=10^{12}$, which is a function of both $T_{i}$ and $\phi_{j} / T_{j}$, can be satisfied by a wide rar te r mameters; however the imposition of the reactor-like condition that

$$
\frac{(n \tau)_{\|}}{n \tau_{0}} \approx \sqrt{\pi}\left(\frac{\phi_{i}}{T_{i}}\right) e^{\phi_{j} / T_{i}}=50 \text {, }
$$

where ${ }^{n} \tau_{0}=? .85 \times 10^{10} \sqrt{A} T_{i}^{3 / 2} \tau_{0}$ is the Pastukhov confinement time, and $A$ is the ion mass number, i.e., that the ion be held on the order of 50 collision times before escaping over the barrier, leads to the solution $T_{i}=1 \mathrm{keV}$ and $\phi_{j} / T_{j}=2.5$.

These values for $n_{c}, T_{i}$, and $\phi_{j} / T_{i}$ are the starting points for our calculations of the remaining parameters. An additional input parameter that is necessary for the calculation is the barrier filling rate, $I_{\text {trap }}$. Logan used a value of 12 amps per plug to calculate the parameters for the positive configuration and, for comparison purposes, we have used 17 amps per plug. This allows for a somewhat greater plug volume in the negative potential case since the peak of the ion potential occurs at the outer mirror rather than near the plug midplane. Refinements of the barrier trapping calculations, which include bounce averaging, indicate that the trapping rate may be a factor of two or more higher, but these calculations are still in a state of flux. Consequentiy, since one of the purposes of this exercise is to compare the positive case with the negative case for the same cold ion confinement parameters, we have used the value of 17 amps/plug, mentioned above. 
In Table 10-1 we list results of solving the steacuy-state equations for the above infut conditions using the TMX(N) magnetir. field parameters for three cases:

- Case A - Resonant transport the same as the positive case.

- Case B - No resonant transport.

- Case C - No resonant transport. TCRH heating of the central-ce11 plasma instead of neutral beams.

Case $B$ is of special interest since resonant transport may be greatly reduced with the reversal of the radial potential gradient (see Sec. 8). We see that there is little difference between the parameters for the positive and negative mode of operation if resonant radial transport is unchanged. The central-cell cold-ion density is somewhat lower for the negative case because of the requirement for runiing the plugs as nigher $g$ values cambined with the 1 imit on totai plug density set by ECRH cutoff. The electron temperature is lower both because of lower input power and somewhat higher e"ectron lass rate. The absence of radial transport reduces the required amount of centralcell beam current, or for the same amount of current, allows a higher centralcell ion temperature, a larger ion ?astukhov confinement parameter and a lowerbarrier filling rate. Replacing the central-cell beams with ICRH would eliminate the hot component of the plasma, assuming bulk heating of the inns, and greatly reduce the total gas load on the plasma. 
Table 10-1. TMX upgrade parameters.

\begin{tabular}{|c|c|c|c|c|}
\hline \multirow[b]{2}{*}{ Parameter } & \multirow{2}{*}{$\begin{array}{l}\text { ThX(P) } \\
\text { Thermal barrier } \\
\text { (reference case } \\
\text { proposal parameters) }\end{array}$} & \multicolumn{3}{|c|}{$T M \times(N)$} \\
\hline & & Case A & Case R & Case C \\
\hline Density, $\mathrm{n}_{\mathrm{c}}\left(10^{12} \mathrm{~cm}^{-3}\right)$ & 23 & 17.8 & 14.0 & 10.6 \\
\hline Ion density, $n_{i c}$ (bulk) $\left(10^{1 ?} \mathrm{~cm}^{-3}\right)$ & 16.7 & 10.6 & 10.6 & 10.5 \\
\hline Ion temperature, $T_{i}(\mathrm{keV})$ & 0.90 & 1.0 & 1.0 & 1.0 \\
\hline Electron temperature, $T_{\text {ec }}(\mathrm{keV})$ & 0.60 & 0.43 & 0.41 & 0.27 \\
\hline Beta, $B_{C}(1)$ & 0.25 & 0.14 & 0.09 & 0.05 \\
\hline Ion-confining potential, $\phi_{i}$ (kev) & $2 . ?$ & 2.5 & 2.5 & 2.5 \\
\hline Electron-ccnfining potential, $\phi_{e}$ (kev) & 2.6 & 2.4 & 2.4 & 1.6 \\
\hline Axial confinement, $(\mathrm{nt})_{\mathrm{c} \|}\left(10^{11^{\mathrm{e}}} \mathrm{cm}^{-3} \mathrm{~s}\right)$ & 10 & 10 & 10 & 10 \\
\hline Radial confinement, $(n \tau)_{1}\left(10^{11} \mathrm{~cm}^{-3} \mathrm{~s}\right)$ & 5 & 2 & $\infty$ & $\infty$ \\
\hline Beam ionization fraction, $f_{\text {ion }}^{c}$ & 0.15 & 0.12 & 0.09 & - \\
\hline Beam charge-exchange fraction, $f_{c x}^{c}$ & 0.30 & 0.23 & 0.18 & - \\
\hline Beam current, $\mathrm{I}_{\text {beam }}^{\mathrm{C}}$ (incident) (arip) & 180 & 131 & 58 & - \\
\hline Gas feed, I gas (H equiv.) (amp) (ionized) & $4 ?$ & 65 & 32 & 38 \\
\hline ICRH power (absorbed) (kW) & - & - & - & 143 \\
\hline Plugs (per plug) & & & & \\
\hline Density, $n_{p}\left(10^{12} \mathrm{~cm}^{-3}\right)$ (at electron feed) & 7 & 7 & 7 & 7 \\
\hline Conf inement, $\left.(\mathrm{Tn})_{\mathrm{a}}\right)_{\mathrm{p}}(\mathrm{cx})\left(10^{10} \mathrm{~cm}^{-3-\mathrm{s}}\right)$ & 5 & - & - & - \\
\hline Perpendicular beta, $\beta_{1}$ & 0.33 & 0.37 & 0.32 & $0,3 ?$ \\
\hline Parallel beta, $\beta_{\| f}$ & 0.01 & $0 . n R$ & $n . n R$ & $0 . n 8$ \\
\hline Barrier electron energy, $\overrightarrow{\mathrm{E}}_{\mathrm{eh}}$ (b) $(\mathrm{keV})$ & 50 & 50 & 50 & 50 \\
\hline
\end{tabular}


SECTION 11

DIAGNOSTICS

(B. Hornady)

The operation of TMX(N) requires evaluation of the adequacy of the diagnostics set which was constructed for TMX(P). In the discussion which follows it will be shown that the present set is adequate for determining many features of any plasma but is somewhat deficient in establishing the axial profile of the potential. The diagnostics and the machine parameter measurements which are installed are listed, the usefulness of each is addressed, and new needs are identified for further consideration.

The diagnostics include machine parameter measuring systems namely: current monitors on each of the seventeen magnet circuits, neutral beam current and voltage monitors, current monitors on the startup guns, gas flow measurement on gas boxes, incident and reflecteo power measurements on each ECRH waveguide, and pressure monitors of machine vacuum both between and during a plasma shot. These monitor systems provide valuable machine information independent of the nature of the operation of TMK.

The diagnostics which will be available initially on TMX-Upgrade are listed in Table 1T-1. In Table 11-2 diagnostics which are currently in development or planning are listed.

The majority of the diagnostics in Table 11-1 will be useful without modification in the negative mode. The SED arrays, determining the angular distribution of the sloshing ions, would not be useful as sloshing ions are not present. The end-cell Thomson scattering system will not be useful since, at the outer 3-T field region, only hot electrons are expected. There electrons doppler spread the ruby laser light over too wide a wavelength range for practical measurements. If the Thomson scattering system were moved to the 1-T field location towards the central cell it might be possible to study the electron feed. Due to the different character of the end 10ss, the ELA as built would not be adequate for determining plug potential. Since the end lass ions are potentially confined, the ion energy distribution is expected to extend down to the potential $\phi_{w}$, the potential drop from $\phi_{i}$ to the wall. These could possibly be used to measure the electron end-loss distribution which would be expected to have a cutoff of ${ }_{e}$, however many processes can perturb electrons (e.g., rf) making sucli measurements difficult. Finaliy, 
because the BAD uses a sloshing beam which would not be present in the negative mode, the detector should be moved to view perpendicular to the axis and a lower power, ronperturbing beam used as the source, A low current leam is desired to avoid unwanted beam trapping in the plug.

of the Diagnostics listed in Table 11-1 onTy the ELA provided information on plasma potential. Thus, even for TMX(P) operation, the diagnostics of Table 11-2 are necessary to determine potentials. Especially useful are the charge exchange analyzer which measures the ion energy distribution at a point, and hence the ambipolar potential a 150 , the plasma potential probe which uses an ion beam to measure local values of the potential in the midplane of the central cell, and the pitch angle end-loss analyzer which measures the ion energy end-loss spectra in the loss cone so that the potential may be determined by the shift in energy of the pump beam injected ions. These diagnostics are desirable for either mode of operation of TMX-Upgrade. Doppler broadening determines the angular rotation direction of the central-cell plasma, hence the sense of the electric field giving at least qualitative information on the ambipolar potential.

From the above considerations it is concluded that the initial diagnostics set (Table 11-1) is sufficient for establishing the major features of the plasma in $T M X(P)$ but requires supplementation by the diagnosties of Table Il in order to determine important details, especially of the potential. In the case of negative operation, $\operatorname{TMX}(N)$, several of the systems in the initial set are no longer viable and the diagnostics of Table 11-2 become essential for determining the nature of the plasma especially the C\%, PPP, and PAELA. Given the installation of the diagnostics of Tables $M, 1$ and $11-2$, the plasmas in either of the two major operation schemes should be wel1measured.

\section{ACKNOWLEDGMENT}

We acknowledge the work of G. Logan, T. K. Fowler, and D. Baldwin in conceiving the ideas and doing much of the detailed work, and the aid of R. Jong during the initial phases of this investigation. 
Table 11-1. TMX-Upgrade initial diagnostics.

\begin{tabular}{|c|c|c|}
\hline Dragnostics & Purpose & Location(s) \\
\hline Beam attenuation detector (BAD) & $n_{e}$ & East plug, central cell \\
\hline Thomson scattering system (TSS) & $T_{e}, n_{e}$ & East plug, central cell \\
\hline Microwave interferometers (MIS) & $n_{e}$ & $\begin{array}{l}\text { East plug, east barrier, } \\
\text { central cell, west plug }\end{array}$ \\
\hline End ioss analyzer (ELA) & $J(E)$ & East and west end walls \\
\hline Extreme ultraviolet (EUV) & Impurities & East plug or central cell \\
\hline Magnetic loops & Plasmia pressure & 14 locations \\
\hline Faraday cups/current collectors & $\begin{array}{l}\text { End-loss current of } \\
\text { ions and electrons }\end{array}$ & East and west end walls \\
\hline $\begin{array}{l}\text { Secondary emission detectors } \\
\text { (SED) }\end{array}$ & $\begin{array}{l}\text { End plug angular } \\
\text { distribution }\end{array}$ & East and west plugs \\
\hline Fast ion gauges $\{F I G\}$ & $\begin{array}{l}\text { Transient gas } \\
\text { pressure }\end{array}$ & $\begin{array}{l}\text { East and west fan } \\
\text { tanks and central ce } 11\end{array}$ \\
\hline RF Probes & Plasma instabilities & $\begin{array}{l}\text { East plug, east Barrier, } \\
\text { west barrier, central cell }\end{array}$ \\
\hline
\end{tabular}


Table 11-2. Planned diagnostics.

\begin{tabular}{|c|c|c|}
\hline Diagnostics & Purpore & Location(s) \\
\hline$x$-rays & $\begin{array}{l}\text { Hot electrons dis- } \\
\text { tribution }\end{array}$ & $\begin{array}{l}\text { East plug, west plug, } \\
\text { Central cell }\end{array}$ \\
\hline Microwave radiometer & $\begin{array}{l}\text { Plug hot electron } \\
\text { distribution and } \\
\text { instabilities }\end{array}$ & East plug \\
\hline Bolometers & Power loss to walls & Throughout system \\
\hline Charge exchange analyzer (CXA) & $\begin{array}{l}\text { Ion energy distri- } \\
\text { bution }\end{array}$ & \\
\hline Doppler broadening & $\begin{array}{l}\text { Impurities temper- } \\
\text { ature, plasma } \\
\text { rotation }\end{array}$ & Central cell \\
\hline Plasma potential probe (PPP) & $\begin{array}{l}\text { Spacially resolved } \\
\text { potential, density, } \\
\text { and temperature }\end{array}$ & [entral cel] \\
\hline $\begin{array}{l}\text { Pitch angle end-loss analyzer } \\
\text { (PAELA) }\end{array}$ & $\begin{array}{l}\text { Ion end-7oss energy } \\
\text { spectra }\end{array}$ & East and west end walls \\
\hline
\end{tabular}


APPENDIX A

START-UP FORMULARY

(D. P. Grubb)

We now present the formulae used to calculate the equilibria presented in Section 9, Start-Up. Symbols are defined in Table Al. Units are included in the equations. Spatial profiles are described in Table A2.

The equations presented in this section describe the giobal (volume-integrated) power and particle balance. Many of these equations were derived by performing volume integrals of local power and particle balance equations. When this was done, geometric constants were introduced into the equations. Where possible, we have set these geometric constants off in 5 p:are brackets, [], so that the reader is aware of their origin. The values presented in this formulary are consistent with the profiles shown in Table A2. However, if the assumed prof $i$ les were to be changed, the geometric factors would also change. We recommend, therefore, that the readei understand the origin of these coefficients to correctly apply these equations to machines other than $T M X\{N\}$.

As an example, we now derive the volume-integrated drag power exchanged between the central-cell hot ions (from beam injection) and the central-cell electrons. A calculation consistent with the profiles given in Table $A 2$ is

$$
\begin{aligned}
P_{D}\left(h i-e^{-}\right)= & \int_{0}^{R} \int_{-L_{m}}^{L_{m}} k q n_{i h}(r, z) \pi_{e c}(r, z) \\
& \frac{\left[E_{j h}(r, z)-3 / 2 k T_{e c}(r, z)\right]}{k T_{e c}^{3 / 2}(r, z)} d V_{c c} \\
= & k q \int_{0}^{R_{c}} \hat{n}_{i h}\left[1-\left(r / R_{c}\right)^{2}\right] \hat{n}_{e c}\left[1-\left(r / R_{c}\right)^{2}\right] \\
& \cdot \frac{\left\{\hat{E}_{i h}\left[1-\left(r / R_{c}\right)^{2}\right]-3 / 2 k \hat{T}_{e c}\left[1-\left(r / R_{c}\right)^{2}\right]\right\}}{\left(k \hat{T}_{e c}\left[1-\left(r / R_{c}\right)^{2}\right]\right\}^{3 / 2}} \\
& \cdot 2 \pi r d r \int_{-L_{m}}^{L_{m}} \frac{d z}{B / \theta_{0}}
\end{aligned}
$$




$$
\begin{aligned}
& =k q \hat{n}_{i h} \hat{n}_{e c} \frac{\left(\hat{E}_{h i}-3 / 2 k \hat{T}_{e c}\right)}{k T_{e c}^{3 / 2}}[2 / 5] \pi R_{c}^{2} L_{e f f} \\
& =q \hat{n}_{i h}\left(\mathrm{~cm}^{-3}\right) \hat{n}_{e c}\left(\mathrm{~cm}^{-3}\right) \frac{\left[\hat{E}_{i h}(k e V)-3 / 2 k \hat{T}_{e c}(k e V)\right]}{5 \times 10^{11} \hat{k T}_{e c}^{3 / 2}(k e V)} \\
& -[2 / 5] v_{c c}\left(\mathrm{~cm}^{3}\right),
\end{aligned}
$$

where $K^{-1}=5 \times 10^{71}$ for $\ell n \Lambda=20$ and hydrogen ions with densities in $\mathrm{cm}^{-3}$ and energies in keV. This is Eq. $(B 2-5)$.

As shown in this example and in Table AI-2, most of the central-cell plasma parameters have been treated as having parabolic radial profiles but constant axial profiles. In the plug, however, we have also included the axial variations of the electron temperatures and densities and the axial variation of the plasma potential. For this reason, most of the coefficients used in the hot-electron power balance had to be derived numerically. The derivation of these geometric constants is included in Ref. $[A T-1]$.

\section{A1. HOT (MIRROR-CONFINED) PLUG ELECTRONS}

The hot electron power and particle balance formulae presented in this section were derived in a separate memo ${ }^{A I-1}$ so we will not rederive them here. We do note, though, that the numerical coefficients in many of the equations were derived from evaluation of volume integrals. Since the assumed profiles (Table $A I-2$ ) are unique to $T M X$, the coefficients are not universally applicable. For this reason the more general volume integrals are also presanted.

\section{A1. 1. Particle Balance.}

$$
[0.47] v_{\text {eh }} \frac{d \hat{n}_{\text {eh }}}{d t}=\frac{1}{e}\left(i_{\text {fue }}-I_{\text {scatt }}\right)
$$

$I_{\text {fuel }}$, the net current of cold electrons trapped by ECRH, are given by

$$
I_{\text {fuel }}=I_{\mu} / \Delta
$$


where $I_{\mu}$ is the total (gross) current of cold electrons trapped by ECRH. A1-2 If we assume that the trapping rate is independent of radius then the following expression agrees with Fokker $-P$ lanck code calculations within $\pm 30 \%$ and

$I_{\mu}(\operatorname{amps})=2.2 \times 10^{-6} \frac{\hat{n}_{e c}\left(10^{12} \mathrm{em}^{-3}\right)}{k \hat{T}_{e c}(\mathrm{keV})} \exp (-0.1 \mathrm{e \Delta \phi} / \mathrm{kT} e c) E_{\mu}^{2}(V / \mathrm{cm})\left[\pi r_{\mu}^{2}(\mathrm{~cm})\right]$

The term $\Delta$ in Eq. [A].1(a)] models the net collisional detrapping of ECRH-trapped electrons by cold-electron, cold-electron collisions, i.e., in order to get a net trapping current of hot electrons equal to I fuel, the gross ECRH trapping current must be $I_{\mu}=\Delta I_{\text {fuel }}$. Analytic fits to Fokker-P lanck code runs at constant rf electric field $\left(E_{\mu}=100 \mathrm{~V} / \mathrm{cm}\right)$ yield a value of delta given by

$$
\Delta=\frac{0.7\left[\mathrm{n}_{\mathrm{ec}}\left(10^{12} \mathrm{~cm}^{-3}\right)\right]^{1 / 2}}{\mathrm{kT}_{\mathrm{ec}}^{3 / 2}(\mathrm{keV})}
$$

evaluated at the fundamentai resonance heating zone.

If we now combine Eqs. (A1.1a), ( $A 1.2)$, and (A1.2a) we find

$$
\begin{aligned}
I_{\text {fuel }}= & 3.1 \times 10^{-6}\left[n_{\mathrm{ec}}\left(10^{12} \mathrm{~cm}^{-3}\right) \exp \left(-0.1 \mathrm{e} \hat{\Delta \phi} / \mathrm{kT} T_{e c}\right)\right]^{1 / 2} \\
& \cdot k F_{\mathrm{ec}}^{1 / 2}(\mathrm{keV}) \pi r_{\mu}^{2}(\mathrm{~cm}) \mathrm{E}_{\mu}^{2}(\mathrm{~V} / \mathrm{cm}) .
\end{aligned}
$$

For this report, we have assumed that $E_{\mu}=100 \mathrm{~V} / \mathrm{cm}$ independent of time ano radial position. Ray tracing calculations will be used in the future to study the radial variations of $E_{\mu}$. When these calculations are analyzed, the radial profile of $E_{\mu}$ will be included in our analys is of start-up and steady-state.

$I_{\text {scatt }}$ is equal to the total hot-e lectron-scattering loss current due to both ion and electron collisions and is given by: 


$$
\begin{aligned}
& I_{\text {scatt }}=2.4 I_{\text {hh }}+2 I_{\text {he }} \\
& =2.4\left(4.8 \times 10^{-5} \int \frac{n_{e h}^{2}}{k T_{e h}^{3 / 2} \log R_{h}^{p}} d v_{e h}\right) \\
& +2 .\left(6.7 \times 10^{-5} \int \frac{n_{e h} n_{e c}}{\left(k F_{e c}+k T_{e h}\right)^{3 / 2} \log R_{l l}^{p}} d v_{e h}\right)
\end{aligned}
$$

In these equations the factor of 2 multiplying $I_{h c}$ is to include ion-electron scattering for the ions which charge neutralize the cold electrons. Similarly the factor of $2.4 \mathrm{limes} \mathrm{I}_{\mathrm{hh}}$ reflects the additional scattering due to ion-electron collisions and includes the fact that the ion-electron scattering rate is $40 \%$ greater than the hot electron-hot electron scattering rate.

Performing the integrals for assumed profiles yields

$$
\begin{aligned}
I_{\text {scatt }}(\operatorname{amps}) & \approx 2.4\left[58 \frac{\mathrm{n}_{\mathrm{eh}}^{2}\left(10^{12} \mathrm{~cm}^{-3}\right)}{\mathrm{E}_{\mathrm{eh}}^{3 / 2}(\mathrm{keV})}\right] \\
& +2\left[\frac{92 \hat{\mathrm{n}}_{\mathrm{ec}}\left(10^{12} \mathrm{~cm}^{-3}\right) \hat{n}_{\mathrm{eh}}\left(10^{12} \mathrm{~cm}^{-3}\right) \exp \left[-0.2\left(\mathrm{e \Delta \phi} / \hat{\mathrm{kT}}_{\mathrm{ec}}\right)^{1 / 2}\right]}{\mathrm{E}_{\mathrm{eh}}^{3 / 2}(\mathrm{keV})}\right] .
\end{aligned}
$$

Note: When the plasma potential is positive, the value of $I_{h c}$ used is twice that given in Eq. (Al.3) because cold electrons are on both sides of the hot distribution, not just learing in from the central-cell side (Fig. 9-2).

A1.2. Power Balance

$$
\frac{3}{2} \text { e }[0.43] v_{\text {eh }} \frac{d}{d t}\left(\hat{n}_{\text {eh }} k \hat{T}_{\text {eh }}\right)=P_{2}+(1-\delta) P_{1}+P_{\text {Fuel }}-P_{\text {scatt }}-P_{\text {cond }}
$$

where,

$P_{2}=\alpha_{2} P\left(\omega=2 \omega_{c e}\right)$ with $\alpha_{2}=1-\exp \left[-1.9 k \hat{\mathrm{T}}_{\mathrm{ec}}(\mathrm{keV}) \hat{n}_{\mathrm{eh}}\left(10^{12} \mathrm{~cm}^{-3}\right)\right]$,

$p_{1}=\alpha_{1} P\left(\omega=\omega_{c e}\right)$ with $\alpha_{1}=1-\exp -\left[\frac{4.4 k \tilde{T}_{e h}(k e v)}{n_{e h}\left(10^{12} \mathrm{~cm}^{-3}\right)}\right]$, 
and $\delta$ (described in Section A2.2) is the fraction of the fundamental ECPH power absorbed by the passing electrons which is subsequentily deposited in the central cell.

The term $P_{\text {fue }}$ is the power input to the plug electrons due to ECRH trapping of the passing electrons and is also presented in Sec. A2.2. Generally, $P_{\text {fuel }}$ is a small term in the hot-electron power balance.

$$
\begin{aligned}
& P_{\text {scatt }} \text { is the power lost due to particle scattering pitch-angle-losses: } \\
& P_{\text {scatt }}=2.4 P_{h h}+2 . P_{h c}
\end{aligned}
$$

which gives

$$
\begin{aligned}
P_{\text {scatt }}(\mathrm{kW}) & =2.4\left[3.7 \times 10^{-3} \int \frac{\mathrm{n}_{\mathrm{eh}}^{2}\left(10^{12} \mathrm{~cm}^{-3}\right)}{\mathrm{E}_{\mathrm{eh}}^{1 / 2}(\mathrm{keV})} d V_{\mathrm{eh}}\right] \\
+ & 2 \cdot\left[2.6 \times 10^{-3} \int \frac{\mathrm{n}_{\mathrm{eh}}\left(10^{72} \mathrm{~cm}^{-3}\right) \mathrm{n}_{\mathrm{ec}}\left(10^{12} \mathrm{~cm}^{-3}\right) \mathrm{E}_{\mathrm{eh}}(\mathrm{keV})}{\left[E_{\mathrm{eh}}(\mathrm{keV})+\mathrm{E}_{\mathrm{ec}}(\mathrm{keV})\right]^{3 / 2}} \mathrm{dV} \mathrm{eh}\right]
\end{aligned}
$$

and

$$
\begin{aligned}
P_{\text {scatt }}(k W) & =2.4\left[\frac{28 \hat{n}_{\mathrm{eh}}^{2}\left(10^{12} \mathrm{~cm}^{-3}\right)}{E_{\mathrm{eh}}^{T / 2}(\mathrm{keV})}\right]+2 \cdot\left\{\frac{32 \hat{n}_{\mathrm{eh}}\left(10^{32} \mathrm{~cm}^{-3}\right) \hat{n}_{\mathrm{ec}}\left(10^{12} \mathrm{~cm}^{-3}\right)}{E_{\mathrm{eh}}^{1 / 2}(\mathrm{keV})}\right. \\
& \left.\cdot \exp \left[-0.46\left(\mathrm{e \Delta \phi} / \mathrm{kT} \mathrm{ec}_{\mathrm{ec}}\right)^{1 / 2}\right]\right\}
\end{aligned}
$$

Again, note that $P_{h c}+2 P_{h c}$ in Eq. (Al.6) if the potential is positive for the same reason that the scattering losses were doubled.

$P_{\text {cond }}$ is the power transferred to the cold electrons and ions. For the purposes of this report we ignome the power transferred to the ions which is smal1. Therefore,

$$
\begin{aligned}
P_{\text {cond }}= & P_{D}\left(h e^{-}-e^{-}\right)=1.1 \times 10^{-2} j n_{e c}\left(10^{12} \mathrm{~cm}^{-3}\right) n_{e h}\left(10^{12} \mathrm{~cm}^{-3}\right) \\
& \cdot \frac{\left[E_{e h}(k e V)-E_{e c}(k e V)\right]}{\left[E_{e h}(k e V)+E_{e c}(k e v)\right]^{3 / 2}} d V_{e h}
\end{aligned}
$$


in $\mathrm{kH}$. Evaluating the integrals gives

$$
\begin{aligned}
P_{\text {cond }}(k W) \simeq & \frac{65 \hat{n}_{e c}\left(10^{12} \mathrm{~cm}^{-3}\right) \hat{n}_{e h}\left(10^{12} \mathrm{~cm}^{-3}\right)}{\hat{E}_{e h}^{T / 2}(k e V)} \\
& \cdot \exp \left(-3.1 \hat{E}_{e c} / \hat{E}_{e h}\right) \cdot \exp \left[-0.73\left(e \Delta \hat{\phi} / k T_{e c}\right)^{0 . \sigma_{1}}\right]
\end{aligned}
$$

Again, this value is multiplied by 2 if the plasma potential is positive.

A2. CENTRAL-CELL ELECTRONS

A2.1. Particle Balance

$$
\left.e[1 / 2] v_{c c} \frac{d}{d t} \hat{n}_{e c}=I_{g} f_{i}^{g}+I_{b}^{c} \Sigma f_{i}^{c}+2 I_{b}^{p} f_{i}^{p}-I_{\text {Past }}-2 I_{\text {Fue }}\right] \text {, }
$$

where the first three terms represent ionization of gas, central-cell beams, and the pump beams, respectively, and are given by

$$
\begin{aligned}
& I_{g} f_{i}^{g} \simeq \frac{\sigma_{i}}{\left(\sigma_{i}+\sigma_{c x}\right)} I_{g} \simeq 1 / 3 I_{g}, \text { and (in the thin plasma limit) } \\
& I_{b}^{c} \Sigma f_{i}^{c}=1.8 \times 10^{-5} \hat{n}_{i c}\left(10^{12} \mathrm{~cm}^{-3}\right)+1.5 \times 10^{-4} \hat{n}_{h i}\left(10^{12} \mathrm{~cm}^{-3}\right) \\
& +2.2 \times 10^{-4} \hat{n}_{e c}\left(10^{12} \mathrm{~cm}^{-3}\right)[1.5] R_{c}(\mathrm{~cm}) I_{b}^{c},
\end{aligned}
$$

and

$$
I_{b}^{p} f_{i}^{p} \simeq 3 \times 1 U^{-4}[2.5] \bar{n}_{e c}\left(10^{12} \mathrm{~cm}^{-3}\right) R_{p}(c m) I_{b}^{p} .
$$

The axial (Pastukhov) losses of electrons are

$$
\begin{aligned}
& I_{\text {past }}=\frac{[2 / 3] \hat{n}_{e c}^{2} \text { e } L_{e f f}^{c} \pi R_{c}^{2}}{(n \tau)_{e c}}, i . e ., \\
& I_{\text {past }}(\text { amps })=\frac{5 \times 10^{3} \hat{n}_{e c}^{2}\left(: 0^{12} \mathrm{~cm}^{-3}\right) R_{c}^{2}(\mathrm{~cm})}{k T_{e c}^{3 / 2}(e V)\left(e \phi_{e} / k T_{e c}\right) \exp \left(e \phi_{e} / k T_{e c}\right)} .
\end{aligned}
$$

$I_{\text {Fuel }}$ is given by Eq. (A1.2b). 
Note: There is no source term for the central-cell electrons proportional to the scattering losses of the hot electrons because the lower outer plug mirror in the TMX field will cause the plug scattering losses to escape to the end wall.

A2.2. Power Balance

$$
\begin{aligned}
& \frac{3}{2} e[1 / 3] v_{\text {cc }} \frac{d}{d t}\left(\hat{n}_{\text {ec }} k \hat{T}_{e c}\right)=P_{0}\left(h i-e^{-}\right)+2 P_{D}\left(h e^{-}-e^{-}\right)+2 P_{\text {pump }} \\
& +2 \delta P_{l}-P_{\text {Past }}-P_{\text {2nd }} e^{-} \\
& \quad-I_{g} f_{i}^{g}(30 \text { eV })-2 P_{\text {Fuel }}-P_{0}\left(e^{-}-i\right)-P_{z},
\end{aligned}
$$

where $P_{D}\left(h e^{-}-e^{-}\right)$is given by $E q .(A 1.7)[2 \times E q .(A 1.7)$ if positive potential],

$$
\begin{aligned}
P_{D}\left(h i-e^{-}\right)= & q[2 / 5] \hat{n}_{i h}\left(\mathrm{~cm}^{-3}\right) \hat{n}_{e c}\left(\mathrm{~cm}^{-3}\right)\left[\hat{E}_{i h}(k e V)-3 / 2 k \hat{T}_{e c}(k e V)\right] \\
& \cdot \frac{v_{c c}\left(\mathrm{~cm}^{3}\right)}{5 \times 10^{\pi} \hat{k T}_{e c}^{3 / 2}(k e V)}
\end{aligned}
$$

i.e.,

$P_{D}\left(h i-e^{-}\right)$in $k W=1.7 \times 10^{-4} \hat{n}_{j h}\left(10^{12} \mathrm{~cm}^{-3}\right) \hat{n}_{\mathrm{ec}}\left(10^{12} \mathrm{cmi}^{-3}\right) \mathrm{R}_{\mathrm{c}}^{2}(\mathrm{~cm})$

$$
\cdot \frac{\left[\hat{E}_{i h}(k e V)-3 / 2 k \hat{T}_{e c}(k e V)\right]}{k T_{e c}^{3 / 2}(k e V)} \text {. }
$$

The term $P_{\text {pump }}$ represents power input due to ionization of the pump beams and is given by

$$
\begin{aligned}
& P_{p \text { pup }}=[1 / 2] 2 I_{b}^{p} f_{i}^{p}(E q . B 2) \hat{e \Delta \phi} . \\
& \delta P_{1} \text { is the absorbed fundamental-ECRH power transferred to the }
\end{aligned}
$$
passing electrons which is subsequently deposited in the central cell. We have iwo ways to calculate this quantity. 
The first method is to calculate the power transferred to the circulating electron current which passes through resonance (2 passes per round-trip through the plug)

$$
\begin{aligned}
P_{\text {circ }} & =2\left(\frac{1}{4} n e \bar{v}\right) \Delta W_{1} \frac{\pi r_{\mu}^{2}}{2}(5 \mathrm{kG}) \\
& =n_{e c}(10 \mathrm{kG}) \mathrm{e}^{-\bar{v}} \frac{1.3 \times 10^{-3} E_{\mu}^{2}(\mathrm{~V} / \mathrm{cm}) L_{B}(\mathrm{~cm})}{B(\mathrm{kG})\left[T_{H}(\mathrm{eV})\right]} \cdot \frac{\pi r_{\mu}^{2}}{2}(5 \mathrm{kG})
\end{aligned}
$$

and

$$
P_{c i r c}(k W)=1.9 \times 10^{-5} r_{\mu}^{2}(5 \mathrm{kG}) \hat{n}_{\text {ec }}\left(10^{12} \mathrm{~cm}^{-3}\right) \exp (-0.1 \mathrm{e} \Delta \phi / \mathrm{kT} \text { ec }) F_{\mu}^{2} \cdot(A 2.6 \mathrm{a})
$$

Alternatively, we can approximate $P_{\text {circ }}$ as

$$
P_{\text {circ }}=\left.\frac{n_{\text {pass }}}{n_{\text {total }}}\right|_{10 k G} \alpha_{1} P\left(\omega=\omega_{c_{1}} \cdot\right. \text {. }
$$

In this formula we have assurred that the total fundamental-ECRH power absorbed is distributed between the trapped and passing electrons according to their relative density. To calculate the heating of the passing electrons by this formula we determine ${ }^{10}$ the passing density by

$$
\begin{aligned}
& n_{\text {pass }} \approx \hat{n}_{e c}\left[\operatorname{erf}\left(0.1 e \Delta \phi / k T_{\rho c}\right)^{1 / 2}\right]^{2} \cdot\left\{\operatorname{erf}\left[\frac{(e \phi e-0.1 e \Delta \phi)}{k T_{e c}}\right]^{1 / 2}\right\} \\
& x \exp \left(-0.1 \hat{e} \Delta \phi / k T_{e c}\right)
\end{aligned}
$$

with

$$
\left.n_{\text {total }}\right|_{10 \mathrm{kG}}=n_{\text {pass }}+[2 / 3] \hat{n}_{\mathrm{eh}},
$$

and $\alpha_{7} P\left(\omega=\omega_{c e}\right)$ yiven by $\left.E q .(A) .5\right)$ using oniy the hot electron parameters. In this report we ise $\mathrm{Eq} .(\mathrm{A2} .7)$. 
To this point, we have calculated the fundamental-ECRH power absorbed by the passing electrons, Howeyer, only a fraction of this power is subsequentiy lost to the central-cell electrons. The fraction deposited in the central cell is given by

$$
\frac{\tau_{\text {transit }}}{\left(\frac{1}{\tau_{\Delta E}}+\frac{1}{\tau_{s t}}\right)^{-1}}
$$

where the transit time through the TMX contral cell is

$$
\tau_{\text {transit }}=\frac{2.2 \times 10^{-5}}{\left[k T_{e c}(\mathrm{eV})\right]^{3 / 2}\left[0.41+3.8 \times 10^{-2} \frac{\mathrm{eA \phi}}{\mathrm{kT}}\right]} .
$$

(the dependence on es\$ikT ec reflects the fact that only electrons with

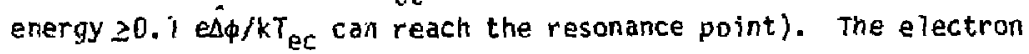
energy relaxation time is

$$
\tau_{\Delta E}=8.4 \times 10^{-9} \frac{\left[2 k T_{e c}\{e y\}+2 \Delta \omega_{1}\right]^{3 / 2}}{\hat{n}_{e c}\left(10^{T 2} \mathrm{~cm}^{-3}\right)},
$$

where $\Delta W_{1}$ is the energy kick the electron gets in passing through the fundamenta $?$ resonance $[$ see $E q .(A 2.6)]$. Finally the pitch-angle trapping time is

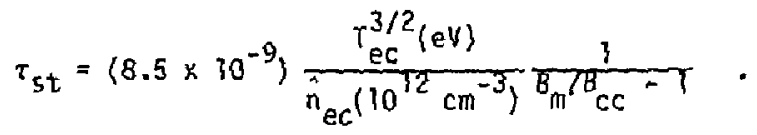

We calculate the power diverted to the central-celi according to

$$
\delta P_{1}=P_{\operatorname{circ}} \frac{\tau_{\text {transit }}}{\left(\frac{T}{\tau_{\Delta E}}+\frac{1}{\tau_{s t}}\right)^{-1}} .
$$

Since both the electron energy-relaxation time and pitch-angle scattering times can be made iong by minimizing the central-cell density during start-up, this potentially large diversion of fundamental ECRH heating power away from the plug electrons can be made smal\} $\left(\sim 10 \%\right.$ of $\left.p_{p}\right)$. 
The Pastukhov loss term in Eq. (A2.4) is evaluated according to

$$
P_{\text {Past }}(k W)=[3 / 5] I_{\text {Past }}(E q . B 2.3)\left[\hat{e}_{e}(k e V)+k \hat{T}_{e c}(k e V)\right] \text {. }
$$

While the plasma potential is still positive during the start-up, we have to include the effects of secondary electrons on the plasma potential and the electron power balance. The term $P_{2 n d} e^{-}$in $E q .(B 4)$ represents this power loss and is givan by

$$
P_{\text {2nd }} e^{-}=\left[I_{P_{a s t}}(E q \cdot B 2 \cdot 3)+2 I_{F_{u e}}(E q \cdot A 2 b)\right][3 / 4] R_{s_{e c}} \hat{T}_{e c}{ }^{\prime}
$$

where

$$
R_{\text {sec }}=\frac{1}{1-\lambda_{s c l}}
$$

with

$$
\lambda_{s c 1}=(0.5 \ln B+0.656)^{1 / 4}
$$

and

$$
B=\frac{k T_{e c}}{e \phi_{e}+q \phi_{i}+k T_{i c}} \text {. }
$$

(The equations for $\lambda_{s c l}$, a measure of the space charge Iimited flow of electrons from the end wall, and B come from Logan; Eq. (48) and Eq. (49) are in the TMX-Upgrade proposal.)

Returning to Eq. (A2.4), we note that the term $I_{g} f_{i}^{g}(30 \mathrm{eV})$ is an approximation to the electron energy loss due to gas fueling which is rough $1 y 30 \mathrm{eV}$ per ionization. This term is small in the final equilibrium but important during start-up.

Similary, the term

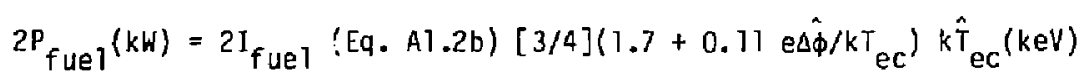

is more important during start-up than the final equilibrium. (Note: The average energy loss per electron of $\left(1.7+0.11 \mathrm{e \Delta \phi} / \mathrm{kT} \mathrm{ec}_{\mathrm{ec}}\right) \mathrm{kT}$ ec was found by analytic fit to several variations of $F_{f u e l} v s \mathrm{e \Delta \phi} / \mathrm{kT}$ ec and reflects the fact that only electrons with energy $z 0.1 \mathrm{es \phi}$ (see Fig. 9-2) can reach the fundamental resonance point.) 
The last term in Eq. (A2.4) is $P_{0}\left(e^{-}-i\right)$, the power transferred from the central-cell electrons to the ions by drag. The expression used is

$$
\begin{gathered}
P_{D}\left(\mathrm{e}^{-}-i\right) \text { in } \mathrm{kW}=3 / 2 \mathrm{q}[2 / 5] \mathrm{V}_{c c} \hat{n}_{\mathrm{ec}}\left(\mathrm{cm}^{-3}\right) \hat{n}_{i c}\left(\mathrm{~cm}^{-3}\right) \\
\cdot\left[\frac{k \hat{j} \mathrm{ec}(\mathrm{keV})-\hat{k}^{\top} \mathrm{ic}(\mathrm{keV})}{5 \times 10^{11} \mathrm{kT}_{\mathrm{ec}}^{3 / 2}(\mathrm{keV})}\right] .
\end{gathered}
$$

A3. CENTRAL-CELL HOT (MIRROR-CONF INED) IONS

A3.1. Particle Balance

$$
\begin{gathered}
\mathrm{q}[1 / 2] v_{c c} \frac{d \hat{n}_{i h}}{d t}=I_{b}^{c} \sum f_{i}^{c}+I_{b}^{c} f_{c x}^{c} \frac{\hat{n}_{i c}}{\hat{n}_{e c}} \\
-I_{D}-I_{\text {scatt }}-[1 / 2] v_{c c}{ }^{q} \frac{\bar{n}_{i h}}{\tau_{c x}^{g}}
\end{gathered}
$$

The source term in this equation, $I_{b}^{c} \Sigma f_{j}^{c}+I_{b}^{c} f_{c x}^{c} \hat{n}_{i c} / \hat{n}_{e c}$, are the ionized beam current (Eq. A2.1) pius the fraction of the beam-particle charge-exchange events which occur with the cold ions:

$$
I_{b}^{c} f_{c x}^{c} \hat{n}_{i c} / \hat{n}_{e c}=4.9 \times 10^{-2} \hat{n}_{i c}\left\{10^{12} \mathrm{~cm}^{-3}\right\} I_{b}^{c} .
$$

The loss terms in Eq. (A3.1) represent drag into the bulk ion population (or the ambipolar hole)--I $I_{D}$, pitch-angle-scattering losses--I scatt, and charge exchange on background gas

$$
-[1 / 2] v_{c c} \frac{n_{i h}}{\tau_{c x}^{g}} q .
$$

The expressions used for these loss currents are:

$$
I_{D}=\frac{[2 / 3] n_{i h q} v_{c C}}{\left(\frac{1}{\tau_{e D}}+\frac{1}{\tau_{i D}}\right)^{-1}} \text {, }
$$


where

$$
\tau_{\mathrm{eD}}=\frac{5 \times 10^{11}}{n_{\mathrm{ec}}(\mathrm{cm})}-\mathrm{kT}_{\mathrm{ec}}^{3 / 2}(\mathrm{keV}) \cdot H \cdot \ln \bar{E}_{\mathrm{inj}} / \mathrm{e}_{\mathrm{L}}
$$

and

$$
\tau_{i D}=\frac{7.6 \times 10^{9}}{\hat{n}_{i c}\left(\mathrm{~cm}^{-3}\right)} E_{i h}^{3 / 2}(\mathrm{keV}) \cdot H \cdot \ln \bar{E}_{i n j} / \phi_{L} .
$$

In these expressions the term $H$ corrects the slowing down time for multiple charge exchange events between the hot ions and the beam noutrals-$H=2$ (see "G" term in Appendix B) and the term in $\bar{E}_{i n j} / e \phi_{L}$ is included because the time we are calculating is for slowing down from the mean injection energy $\left(E_{i n j}\right)$ to the final loss energy ( $\left.\phi_{L}\right)$ where the "loss" energy is given either by $q \phi_{i}$ or $e \phi_{e} / R_{\| l}^{C}-1$ depending upon whether the low-energy ions are electrostatically confineil or whether there is an ambipolar hole in velocity space.

The ion-ion pitch-angle scattering term is similar to the drag terms and is given by

$$
I_{\text {scatt }}=\frac{[2 / 3] \hat{n}_{i h}\left(\mathrm{~cm}^{-3}\right) q v_{c c}}{\tau_{i i}},
$$

where

$$
\tau_{i j}=\frac{2.5 \times 10^{11}}{n_{i c}\left(\mathrm{~cm}^{-3} z_{i}^{2} z_{i}^{2} \ln \Lambda_{i i}\right.} E_{i h}^{3 / 2} \sqrt{A} \cdot H .
$$

The last term in Eq. (A3.1) is charge-exchange losses

$$
I_{g} f_{c x}^{g} \frac{\overline{n_{i h}}}{\bar{n}_{e c}}=\frac{[1 / 2] \hat{n}_{j h} q V_{c c}}{\tau_{c x}^{g}} \text {. }
$$

Since the charge-exchange time is dependent upon the number of beams used, the amount of fueling gas, the location of the gas box or puffer value, and $t ! e$ vacuum conditions, we have left $\tau_{c x}^{g}$ as a free parameter for now. 


\section{A.3.2, Power Balance}

$$
\begin{aligned}
& q[1 / 3] v_{c c} \frac{d}{d t}\left(\hat{n}_{i h} \hat{E}_{i h}\right)=I_{b}^{c}\left[\left(\Sigma f_{j}^{c}\right)+f_{c x}^{c} \hat{n}_{i c} / \hat{n}_{e c}\right] \bar{E}_{i n j} \\
& +I_{b}^{c} f_{c x}^{c} \frac{\hat{n}_{i h}}{\hat{n}_{e c}}\left(\bar{E}_{i n j}-[1 / 2] \hat{E}_{i h}\right)-I_{D} e_{L}-I_{s c a t t} \bar{E}_{L}-[1 / 3] \frac{\hat{n}_{i h}}{T_{c x}^{g}} V_{c c} \hat{E}_{i h} \\
& -P_{D}(h i-i)-P_{D}\left(h i-e^{-}\right) .
\end{aligned}
$$

In this equation power is input by ionization of the central-cell beams and by charge exchange between beam neutral and coldi ions. Beam-neutral charge exchange of a hot ion is either heating or cooling depending on whether the average injection energy is greater than or less than che average hot-ion energy.

The hot-ion power losses in Eq. (A3.9) are due to drag into the cold-ion class of particles [see Eq. (A.3.3)], to scattering loss, to charge exchange with gas, and to drag with the cold ions and the electrons.

These last two terms are given by

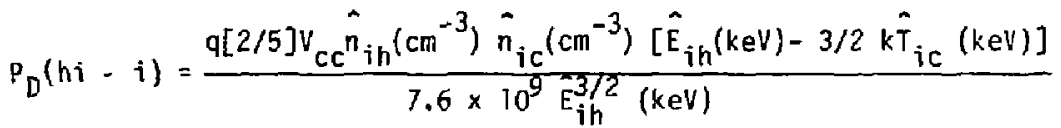

and

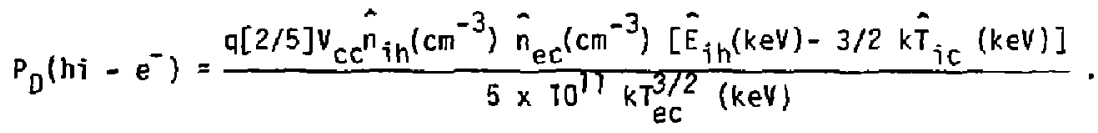

Note: In the scattering loss term we have chosen

$$
\bar{E}_{L}=[1 / 2]\left(\frac{40 \sqrt{A} \Omega_{n A} j}{\ell M_{e i}}\right)^{2 / 3} k \hat{T}_{e c}
$$

that is $1 / 2$ the energy at which the electron drag rate equals the pitch-angle scattering rate. 


\section{A3.3. Quasi-Linear Stream Requirement}

During the second phase of start-up, when the central-cell beams are turned on, a potential drop deveiops between the midplane of the central-cell and the inner mirror. During this phase, then, the mirror-confined central-cell ions may become DCLC unstable. The estimated stream current density required to maintain marginal stability is given by

$$
J_{Q L}\left(A / \mathrm{cm}^{2}\right)=\frac{1.9 \times 10^{-11} \hat{n}_{i h}\left(\mathrm{~cm}^{-3}\right) \hat{\mathrm{kT}}_{\mathrm{eC}}^{3 / 2}(\mathrm{k \in V})}{\left(\mathrm{r}_{\mathrm{c}} / \mathrm{a}_{\mathrm{j}}\right)^{4 / 3} \sqrt{\mathrm{A(amU})} \hat{E}_{i h}(\mathrm{keV})\left(\mathrm{R}_{\|}^{C}-i\right)} .
$$

We note, however, that the required stream current density should be less than this because the central-cell beams are injected at $60^{\circ}$ and will (to some extent) form a sloshing ion distribution.

A4. CENTRAL-CELL COLO IONS

\section{A4.1. Particle Balance}

$$
\begin{aligned}
& q[1 / 2] v_{c c} \frac{d}{d t} \hat{n}_{i c}=I_{g} \stackrel{g}{i}^{g}+I_{g} f_{c x}^{g} \bar{n}_{i h} / n_{e c}+I_{D}+2 I_{b}^{p} f_{i}^{p} f_{c i r c} \\
& -I_{b}^{c} f_{c x}^{c} \hat{n}_{i c} \hat{n}_{e c}=I_{E L}-I_{r a d} \\
& -2 I_{b}^{p} f_{c x}^{p}\left(1-f_{c i r c}\right)
\end{aligned}
$$

The source terms in Eq. (A4.1) are ionization of gas, gas charge exchange of hot ions, 5 lowing down of the beam injected ions into the cold ion population, and the creation of circulating ions from the pump beams. This last term reflects the fact that some of the one-third and one-haif energy components of the pump beams may become trapped between the outboard mirrors or potential maxima and circulate throigil the central cell because they have insufficient parallel energy to escape the confining potential. During start-up $f_{\text {circ }} \simeq 0$, but may be as large as one-half for the final equilibrium presented in Section 10. Code work is presently under way to 
determine the analytic dependence of $f_{c i r c}$ on $e \Delta \phi / k T_{e c}(z)$. In this section (start-up) we assume that $f_{\text {circ }}=0$.

The first loss term in Eq. (A4.1) represents the Toss of a cold ion (and the creation of a hot ion) due to charge exchange between a beam neutral and a cold ion. Similarly, the last term is the pumping of the central-cell ions (both trapped and passing) in the barrier region.

$$
f_{c x}^{p}=1-\exp \left(-\int n_{d i} \text { covs } c x^{\prime} / v_{b}\right)=\left[3.2 \times 10^{-3}\right] R_{p} \hat{n}_{i p}\left[10^{12} \mathrm{~cm}^{-3}\right) .
$$

The remaining two loss terms are due to radiai, $I_{r a d}$, and axial, ' $E$, loss currents. In deriving the start-up scenario we assumed that $I_{\text {rad }}=0$.

The axial loss current is given by

$$
I_{E L}=\frac{[1 / 3] n_{i c}^{2} v_{i c} q}{(n \tau)_{i c}} \text {. }
$$

where

$$
\begin{aligned}
& (n \tau)_{i c}=\left\{2.1 \times 10^{6}\left[\mathrm{kT}{ }_{i c}(\mathrm{eV})\right]^{1 / 2} \mathrm{q \phi}_{\mathrm{i}}(\mathrm{eV})\left[\frac{\mathrm{A(amu})}{2}\right]^{1 / 2}\right. \\
& \left.+\frac{1.3 \times 10^{-6} n_{i c}\left(\mathrm{~cm}^{-3}\right) L_{e f f}^{c}(\mathrm{~cm}) B_{m} / B_{c c}}{\left[\frac{k T_{i c}(e V)}{A(a m u)}\right]^{1 / 2}}\right\} \exp \left(q \dot{\phi}_{i} / k T i c\right)(A 4.3)
\end{aligned}
$$

when the cold ions are electrostatically confined and

$$
\begin{aligned}
& (n)_{\mathrm{ic}}=6.2 \times 10^{5} \mathrm{kT}_{\mathrm{ic}}^{3 / 2}(\mathrm{eV})[\mathrm{A}(\mathrm{amu})]^{1 / 2} \log \mathrm{B}_{\mathrm{m}} / \mathrm{B}_{\mathrm{CC}}
\end{aligned}
$$

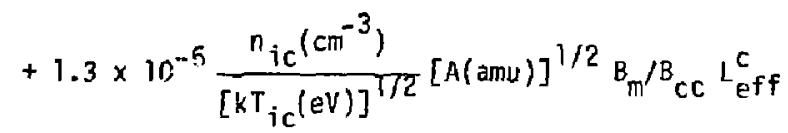

when the cold ions are only magnetically confined $\left(\phi_{i}=0\right)$. Note that in both equations we have only included the cold ion density, $n_{i c}{ }^{*}$ This reflects the fact that both Eqs. (A4.3) and (A4,4) describe scattering due to collision between ions. Since the scattering rate is proportional to $1 / T^{3 / 2}$, the cold ion scattering rate due to collisions with hat ions is less than the cold ion-cold ion scattering rate by a factor of roughiy 


$$
\left(\frac{n_{i h}}{n_{i c}}\right)\left(\frac{T_{i c}}{T_{i h}}\right)^{3 / 2}
$$

that is a small term for the cases studied here.

A4.2. Power Balance

$$
\begin{aligned}
& \frac{3}{2} q[T / 3] v_{c c} \frac{d}{d t} \hat{n}_{i c} k \hat{T}_{i c}=P_{D}(h i-i)+P_{D}(e-i)+\lceil 3 / 5] I_{D} \hat{q \phi}_{i} \\
& +P_{\text {circ }}+P_{\text {ICRH }}+P_{\nabla_{\phi}} \cdot P_{\nabla T} \\
& -3 / 2[1 / 2] \frac{\hat{n}_{i}}{\hat{n}_{e c}} f_{c x}^{g} I_{g} k \hat{T}_{i c}-\frac{3}{2}[1 / 2] \frac{\hat{n}_{i c}}{\hat{n}_{e c}} I_{b}^{c} f_{c x}^{c} k \hat{t}_{i c} \\
& -2[1 / 2](3 / 2) f_{c x}^{p} I_{b}^{p} k \hat{i}_{i c}-[2 / 5] \pi R_{c}^{2} \hat{j}_{E L}\left(e \hat{\phi}_{i}+k \hat{J}_{i c}\right)
\end{aligned}
$$

$P_{D}(h i-i)$ is given by $E q .(A 3.10) . P_{D}(e-i)$ may be positive or negative and is given by Eq. (A2.16). [3/5] $l_{D} q \phi_{j}$ is the power input to the cold ions due to slowing down of the hot ions into the cold ion class of particles (see Eq. A3.3). The term $P_{c i r c}$ is the power input to the central-cell ions due to the trapping of the pump beam ians and is given by

$$
P_{\text {circ }}=2 I_{\text {circ }}\left[E_{\text {circ }}-[1 / 2] q \Delta \hat{\phi}\right] .
$$

The terms $P_{\text {ICRH, }} P_{\square / \phi^{2}}$ and $P_{\nabla T}$ are, respectively, the absorbed ion-cyclotron wave-heating power (from an oscillator coupled to an antenna or from instabilities), the power gaired (or lost) due to radial transport in the presence of a radial, otential gradient, and the convective pnwer loss due to radial particle transpo't. In this section, these terms have all been set equal to zero.

$$
\frac{3}{2}[1 / 2] \frac{\hat{n}_{i c}}{\hat{n}_{e c}} f_{c x}^{g} I_{g} k \hat{T}_{i c}
$$

is the power loss due to charge exchange on background and fueling gas and 


$$
\frac{3}{2}[1 / 2] \frac{\hat{n}_{i c}}{\hat{n}_{e c}} I_{b}^{c} f_{c x}^{c} k \hat{T}_{i c}
$$

is the power loss associated with the loss of i cold ion due to charge exchange between an energetic-beam neutral and the cold ion. Similarly $2[1 / 2](3 / 2) f_{\mathrm{Cx}}^{\mathrm{p}} \mathrm{I}_{\mathrm{b}}^{\mathrm{P}} \mathrm{kT} \hat{\mathrm{T}}_{\mathrm{ic}}$ is the power loss due to pumping ions out of the barrier (both trapped and passing ions). And, finally, $[2 / 5] \pi R_{C}^{2} \hat{j}_{E L}\left(\hat{\phi}_{j}+k \hat{T}_{i c}\right)$ is the power loss associated with ion axial particle loss (see Eq. A4.2).

A5. PLASMA POTENTIAL

\section{A5.1. Phase 1 and 2}

A5.1.1. Plug potential dip, $\Delta \phi$. We determine the potential drop between the central-cell and the plug midplane $[\Delta \hat{\phi}$, see fig. 9-2, note that $\Delta \phi$ as used here has a different meaning from that used earlier), by analogy to a simple tandem mirror

$$
e \Delta \hat{\phi}=\hat{T}_{i c} \ln \left(\hat{n}_{p} / \hat{n}_{c}\right) \text {, }
$$

where

$$
\hat{n}_{p}=\hat{n}_{e h}+\hat{n}_{e c} \exp \left\{-\Delta \hat{\phi} / T_{e c}\right\} .
$$

Therefore,

$$
e \hat{\phi}=\tilde{T}_{i c} \ln \left[\frac{\hat{n}_{\text {eh }}}{n_{e c}}+e^{-\hat{\Delta \phi} / T_{e c}}\right] .
$$

A5.1.2. Central-cell potential, $\Phi_{e}$. The central-cell potential is calculated by requiring quasi-neutralitu. i.e., $n_{e c}=\Sigma n_{i}$. The electron density is given by

$$
\hat{n}_{e}=\left(I_{g} f_{i}^{g}+I_{b}^{c} f_{i}^{c}+2 I_{b}^{p}\right)\left[1+R_{s e c}\right] \cdot \frac{\tau e c}{[172] V_{c c}} .
$$


Setting Eq. (A5.3) Equal to $\Sigma n_{j}$ and solving for $\tau_{e c}$ yields

$$
\tau_{e c}=\frac{\left(n_{i c}+n_{i h}\right)[1 / 2] v_{c c}}{\left(I_{g} f_{i}^{g}+I_{b}^{C_{f}} f_{i}+2 I_{p} f_{i}^{p}\right)\left[1+R_{s e c}\right]},
$$

where we have assumed that the density of passing ions from the pump beams is sma11. In Eq. (A5.4),

$$
\left.\tau_{\text {ec }}=\left[\frac{1}{\tau_{\text {ec }}} \text { (Pastukhov }\right)+\frac{1}{\tau_{\text {fue } 1}}\right] \text {, }
$$

where

$$
\begin{aligned}
& \tau_{e c}(\text { Pastukfiov })=\frac{j}{n_{e c}} \frac{1}{2} \tau_{g 0}^{e e} g\left(R_{\| l}\right) \frac{e \phi_{e}}{k T_{e c}} \exp \left\langle e \phi_{e} / k \tau_{e c}\right\rangle \\
& \tau_{\mathrm{ec}}=\frac{2.6 \times 10^{4} \mathrm{kT}_{\mathrm{ec}}^{3 / 2}(\mathrm{eV})}{n_{\mathrm{ec}}\left(\mathrm{cm}^{-3}\right)} \frac{\mathrm{e} \mathrm{e}_{\mathrm{e}}}{\mathrm{kT}_{\mathrm{ec}}} \exp \left(\mathrm{e \phi} / \mathrm{kT}_{\mathrm{ec}}\right)
\end{aligned}
$$

and

$$
\tau_{\text {fuel }}=\frac{[1 / 2] n_{c} \pi R_{c}^{2} \text { e } L_{\text {eff }}^{c}}{2 I_{\text {fuel }}\left[\text { Eq. A }{ }^{2} b\right]}
$$

Equations (A5.4) through (A5.7) are solved numerically to determine $\phi_{\mathbf{e}}$.

A5.2. Phase 3

A5.2.1. Plug potential dip, $\phi_{e}$. The quasi-neutrality code discussed in Section 2 is used to determine the drop between the central-cell and the plug midplane, ${ }^{2} \phi_{e}=\Delta \phi$ (see Fig. 9-7), again, $\Delta \phi$ has a different meaning here than as used in the body of the report).

A5.2.2. Central-cell potential, $\Phi_{\uparrow}$. Again, we invoke quasi-neutrality to determine the plasma potential. During phase 3 , however, we use the calculated value of $\phi_{e}$ (above) to calculate $\phi_{i}$ as follows: $\Sigma n_{i}=n_{e c}$, i.e., 


$$
\begin{aligned}
& {\left[I_{g} f_{i}^{g}+1_{D}(E q \cdot C 3)+I_{g}^{f} f_{c x}^{g} \frac{\vec{n}_{i h}}{n_{e c}}+2 I_{b}^{p_{f}} p_{f f} \text { irc }\right] \cdot \frac{\tau_{i c}}{[1 / 2] q V_{c c}}} \\
& +n_{i h}=\frac{\left(I_{g} f_{i}^{g}+I_{b}^{c_{f}} c_{i}+2 J_{b}^{p_{f}} p_{i}\right)}{[I / 2] e V_{c c}} \cdot \tau_{e c}(E q \cdot A 5.5),
\end{aligned}
$$

where we have (again) assumed that the density of passing ions from the pump beams is negligble. In Eq. (A5.8),

$$
\tau_{\text {ic }}=\left[\frac{1}{\tau_{11}}+\frac{1}{\tau_{1}}+\frac{1}{\tau_{p m p}}+\frac{1}{\tau_{c \times b}}\right]^{-1},
$$

where $\tau_{\|}$is calculated according to Eq. (A4.4),

$$
\begin{gathered}
\tau_{\perp}=\frac{[1 / 2] \hat{n}_{i c} q_{c c}}{I_{r a d}}, \\
\tau_{p m p}=\frac{[1 / 2] \hat{n}_{i c} q V_{c}}{2 I_{p} f_{c x}^{p}\left(1-\cdot f_{c i r c}\right)},
\end{gathered}
$$

and

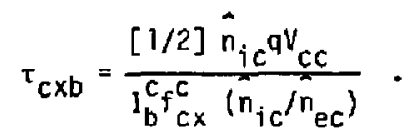

Equation (A4.4) is a function of $\phi_{j}$ so Eqs. (A5.8) through (A5.12) can be combined to solve (numerically) for $\phi_{i}$. 
Table Al. Symbols used in formulary (starting with Appendix A).
$R_{p}$
- parabolic cut-off radius in plug
$L_{\text {eff }}^{p}$
$-L_{-m} \frac{d z}{B / B_{0}}$, for plug $=213 \mathrm{~cm}$
$\mathrm{L}_{\mathrm{m}}$
- Distance from midplane to mirror throat
veh
- $\pi r_{j}^{2} L_{e f f}$
$I_{\text {fuel }}$
- The net current of cold electrons trapped by ECRH
$I_{\text {scatt }}$
- Scattering loss current
$\mathrm{I}_{\mathrm{hh}}$
- Scattering loss current of hat electrons due to hot- electron, hot-electron scattering
$I_{\text {hc }}$
- Scattering loss current of hot electrons due to hot-
electron, cold-electron scattering
neh
- Density of hot electrons
kTeh
- Temperature of hot electrons
$\mathrm{R}_{\text {II }}^{\mathrm{p}}$
- Plug mirror ratio = 4
$I_{\mu}$
- Totat (gross) current of cold e lectrons trapped by ECRH
$\Delta$
- Models the collisional detrapping of ECRH-trapped electrons by cold-electron collisions
$k T_{\text {ec }}$
- Central-cell electron temperature
nec
- Central-cell electron density
$\mathrm{E}_{\mu}$
- Fundamental ECRH electric field
$\Delta \phi$
- Potential drop from central-cell to plug midplane 
Table A1. (cont inued)

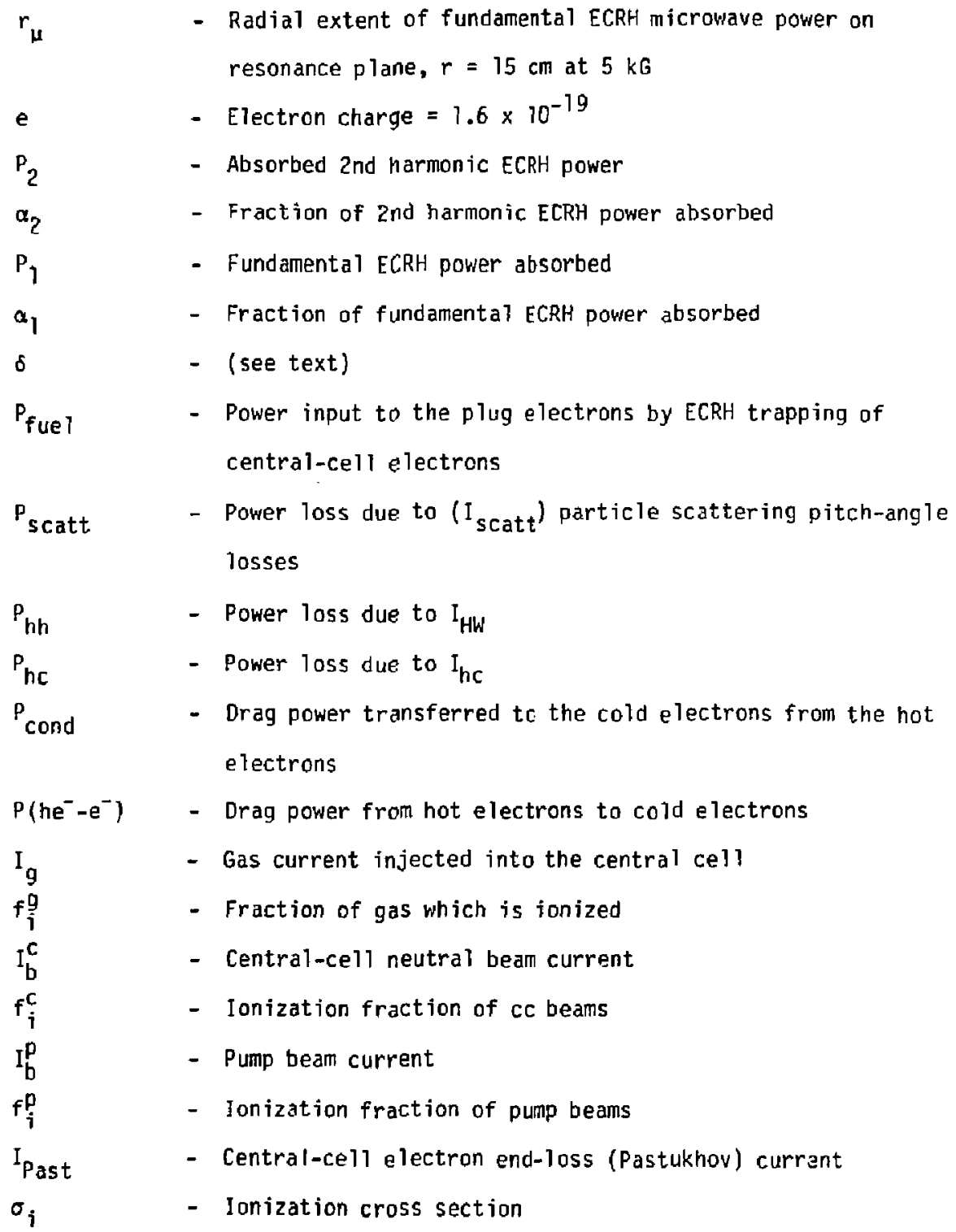


Tab?e 1. (continued)

\begin{tabular}{|c|c|}
\hline$\sigma_{\mathrm{cx}}$ & - Charge exchange cross section \\
\hline$n_{i c}$ & - Central-cell cold-ion densitj \\
\hline$n_{\text {in }}$ & - [entra]-cell hot-ion (mirror-confined) density \\
\hline$R_{c}$ & - Parabolic cut-off radius in central-cell \\
\hline$L_{e f f}^{c}$ & $-\int_{-L_{m}}^{m} \frac{d z}{B / B_{0}}$, central-cell length $=508 \mathrm{~cm}$ \\
\hline$V_{c C}$ & $=\pi R_{c}^{2} L_{e f f}^{c}$ \\
\hline$\phi_{e}$ & - Electron-confi ng potential \\
\hline$P_{0}\left(h i-e^{-}\right)$ & - Drag power from central-cell hot-ions to electrons \\
\hline$P_{\text {pump }}$ & $\begin{array}{l}\text { - Electron power input due to ionization of pump beams at } \\
\phi=0\end{array}$ \\
\hline$P_{\text {2nd }} \mathrm{e}^{-}$ & - Power loss due to secondary electron emission from end walls \\
\hline$P_{0}\left(e^{-}-i\right)$ & - Drag power from cc electrons to ions \\
\hline$P_{Z}$ & - Impurity radiation power \\
\hline$P_{\text {circ }}$ & $\begin{array}{l}\text { - Power input to the central-cell ions due to the trapping of } \\
\text { the pump beam ions }\end{array}$ \\
\hline$L_{B}$ & $\begin{array}{l}\text { - Magnetic scale length near fundamental ECRH resonance at } \\
B=B_{R}, 40 \mathrm{~cm}\end{array}$ \\
\hline nass & - Passing electron density at $B={ }_{k}$ \\
\hline$n_{\text {total }}$ & - Total electron density at $B=B_{R}$ \\
\hline $\operatorname{erf}(x)$ & - Error function of $x$ \\
\hline$\tau_{\text {transit }}$ & - Transit time through the TMX central cell \\
\hline
\end{tabular}


Table A1. (continued)

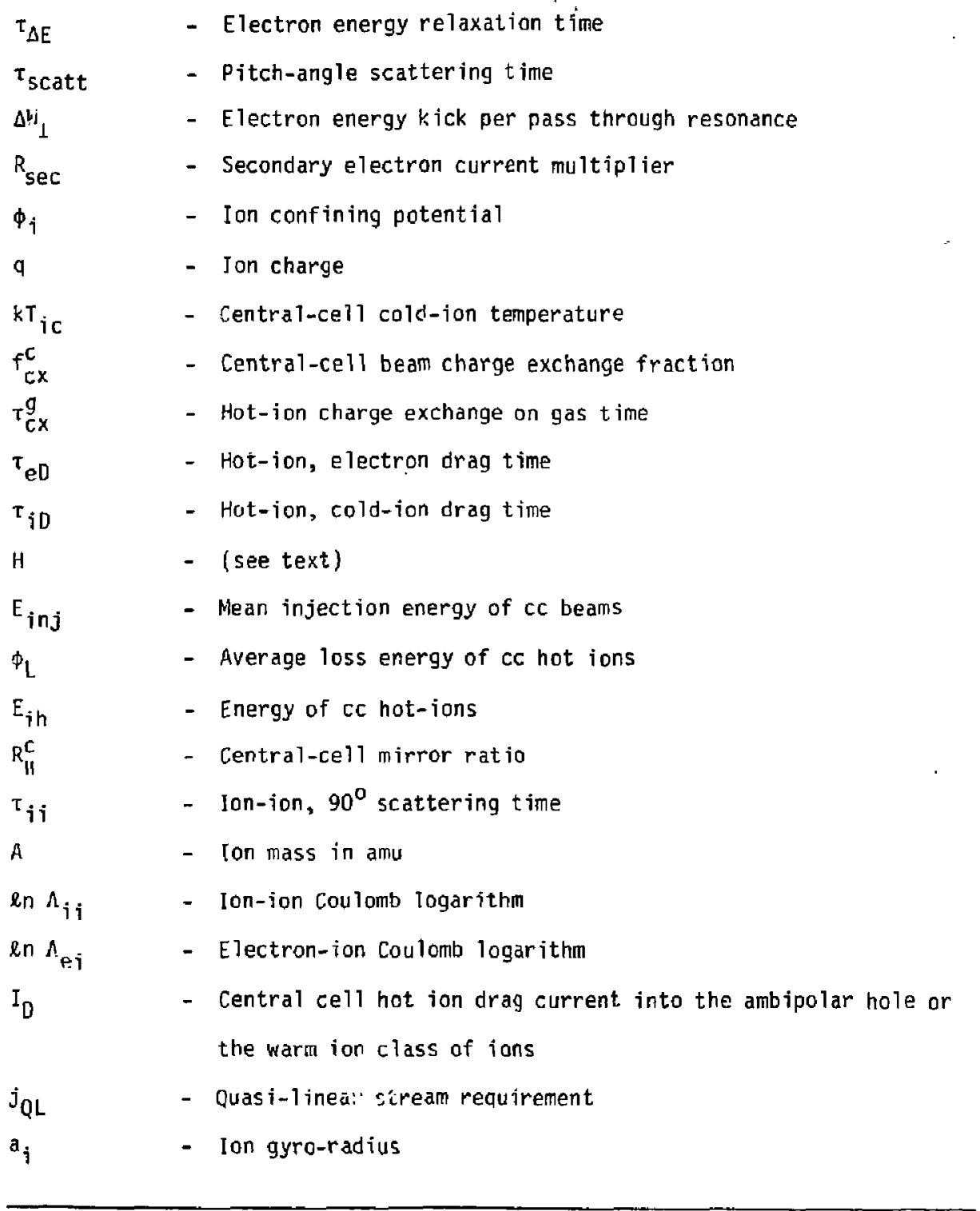


Table A1. (continued)

$\begin{array}{ll}\tau_{s t} & - \text { Pitch-angle trapping time } \\ f_{c i r c} & - \text { Fraction of pump beam ions that are trapped in machine } \\ I_{E L} & - \text { Cold-ion end loss current } \\ I_{\text {rad }} & - \text { Cold-ion radial loss current } \\ \Pi_{p i} & - \text { Plug ion density } \\ B_{m} & - \text { Magnetic field at inner-mirror throat } \\ P_{I C R H} & - \text { Ion rf-heating power } \\ E_{C i r c} & - \text { Average energy of trapped pump-beam ion } \\ P_{\nabla \phi} & - \text { Central-cell ion power gain/loss due to radial transport } \\ & - \text { across radial gradient of } \phi \\ P_{\nabla T} & - \text { Central-cell ion power transport due to particle transport } \\ & \text { across radial ion temperature gradient } \\ g\left(R_{\| 1}\right) & \sqrt{\pi}\left[\left(2 R_{\|}+1\right) / 4 R_{11}\right] \text { en }\left(4 R_{11}+2\right)\end{array}$


Table A2. Spatial profiles.

Note: In a 11 cases, a ( $\left.{ }^{*}\right)$ mark above a parameters means the value of that parameter at $r=0$.

\section{a. End cell}

- $\left.B(r, z)=B_{0}\left[1+z^{1} / 115\right)^{4}\right] ; B_{0}=5 k G$ and

$z^{\prime}=\left|z-z_{0}\right|$ where $z_{0}=567$ is the plug midplane.

- $n_{e h}(r, z)=\hat{n}_{e h}\left[1-\left(r / R_{n e h}\right)^{2}\right]\left[\frac{B_{m}-B(r, z)}{B_{m}-B_{0}}\right]$;

$R_{\text {neh }}=15 \mathrm{~cm}$ and $B_{m}=20 \mathrm{kG}$

- $\left.T_{e h}{ }^{*}, z\right)=\tilde{T}_{e h}\left[r-\left(r / R_{T e h}\right)^{2}\right]\left[a+b z^{\prime}+c z^{\prime 2}+d z^{\prime 3}\right]$;

$\star a=0.98, b=4.46 \times 10^{-3}, c=-7.86 \times 10^{-5}$,

$d=5.62 \times 10^{-8}$ for $z^{\prime} \varepsilon[0,150]$,

and $R_{\text {Teh }}=1000 \mathrm{~cm}$.

- $\frac{\Delta \phi}{T_{e c}}(r, z)=\frac{\Delta \hat{\phi}}{T_{e c}}\left[a_{1}+b_{1} z^{\prime}+c_{1} z^{\prime 2}+d_{1} z^{\prime 3}\right]$;

where $\Delta \phi / T_{\text {ec }}$ is the normalized change in the potential from the central-cel1 potential

$$
\begin{aligned}
* *_{a_{1}} & =1.02, b_{1}=-5.56 \times 10^{-3}, c_{1}=-7.01 \times 10^{-5} \\
d_{1} & =4.14 \times 10^{-7} \text { for } z^{\prime} \varepsilon[0,150] .
\end{aligned}
$$

*Coefficients a-d are from fit to Fokker-Planck code run. **Coefficients $a_{1}-d_{1}$ are from fit to cuasi-neutrality code (Section II) 
Table A2. (continued)

- $\frac{\Delta \phi}{T_{e c}}\left(B=B_{r}\right)=0.1 \frac{\Delta \hat{\phi}}{T_{e c}}$,

i.e., the normalized potential drop from the inner mirror throat $(z=150)$ to the fundamental resonance magnetic field $\left(B_{R}\right)$ is one-tenth of the drop to the midplane of the plug $\left(\Delta \hat{\phi} / T_{\mathrm{ec}}\right)$.

- $n_{e c}(r, z)=\hat{n}_{e c}\left[1-\left(r / R_{n e c}\right)\right]^{2} \exp \left[-e \Delta \phi / k \Gamma_{e c}\right] ;$

$R_{\text {nec }}=25 \mathrm{~cm}$ and $\Delta \phi=\phi_{c c}-\phi(r, z)$

- $T_{\text {ec }}(r, z)=\hat{T}_{e c}\left[1-\left(r / R_{T e c}\right)^{2}\right], R_{T e c}=25 \mathrm{~cm}$

- $n_{i p}(r, z)=n_{e h}(r, z)+n_{e c}(r, z)$

- $T_{i p}(r, z)=T_{i p}\left[1-\left(r / R_{T i p}\right)^{2}\right], R_{T i p}=25 \mathrm{~cm}$

- Pump beari injection angle $=18^{\circ}$

- $V_{e h}=\pi r_{\mu}^{2} L_{e f f}^{p}, r_{\mu}=15 \mathrm{~cm}, L_{e f f}^{p}=213 \mathrm{~cm}$ 
b. Central Ce11

- $n_{e c}(r, z)=n_{c}(r, z)=n_{i h}+n_{i c}=\dot{n}_{e c}\left[1-\left(r / R_{n e c}\right)^{2}\right]$,

$R_{\text {nec }}=32 \mathrm{~cm}[=\sqrt{5 / 3}(25)]$

- $T_{e c}(r, z)=\hat{T}_{e c}\left[1-\left(r / R_{T e c}\right)^{2}\right]$,

$\mathrm{R}_{\text {Tec }}=32 \mathrm{~cm}$

- $n_{i c}(r, z)=\hat{n}_{i c}\left[1-\left(r / R_{i c}\right)^{2}\right]$,

$R_{i c}=32 \mathrm{~cm}$

- $T_{i c}(r, z)=\hat{T}_{i c}\left[1-\left(r / R_{T i c}\right)^{2}\right]$,

$R_{\text {Tic }}=32 \mathrm{~cm}$

- $n_{i h}(r, z)=\hat{n}_{i h}\left[1-\left(r / R_{n i h}\right)^{2}\right]$,

$R_{n i h}=32 \mathrm{~cm}$

- $E_{i h}(r, z)=\hat{E}_{i h}\left[1-\left(r / R_{T i h}\right)^{2}\right]$,

$\mathrm{R}_{\text {Tih }}=32 \mathrm{~cm}$

- Central cell beam injection angle $=60^{\circ}$ 
Table 2. (cont inced)

- $\left.(n \pi)_{e c}=\hat{(n r}\right)_{e c}$

- $\phi_{e} / T_{e c}=\hat{\phi}_{e} / T_{e c}$ - independent of $r$

- $V_{c c}=\pi R_{c}^{2} L_{e f f}^{c} ; R_{c}=32 \mathrm{~cm}$ and $L_{\text {eff }}^{c}=508 \mathrm{~cm}$

- $\phi_{e}(r)=\dot{\phi}_{e}\left[1-\left(r / R_{\phi}\right)^{2}\right]$,

$$
R_{\phi}=32 \mathrm{~cm}
$$

- $j_{E L}(r)=j_{j}(r)=j_{e}(r)=\hat{j}\left[1-\left(r / R_{j}\right)^{2}\right]$,

$$
R_{j}=32 \mathrm{~cm}
$$

- $R_{\text {sec }}=\hat{R}_{\text {sec }}$ - independent of $r$

$-152-$ 


\section{APPENDIX B \\ CENTRAL-CELL STEADY STATE PARTICLE AND POWER BALANCE \\ IN NEGATIVE POTENTIAL GPERATION \\ (W. Nexsen)}

In solving the particle and power balance equations to generate the parameters for $T M X-U$ in the negative patential mode of operation we make a number of simplifications and assumptions. We assume that gas recycling from the end chambers can be controlled and set this component equal to zero. We assume flat ion and electron temperature profile with $T$ boundary $=T_{i}(0)$ and a parabolic density distribution. We assume that the gas feed will be localized in the central-celt mirror throat whwere the mirror contained hot ion density will be low gas and ignore the charge exchange loss of hot ions on cold gas. We estimate that the eiectron current required to feed the hot electron density will be only a small fraction of the total loss current and set it equal to zero. We assume that none of the ECRH power is transferred to the central-cell electrons and only half of the pump beam ionization occurs inside the negative potential peak and at an average energy of $\phi_{e} / 2$. We assume that $P_{\Delta \phi}$ is given by the resanant transport power term and that $P_{v u v}$, $P_{f u e l}$ and $P_{\nabla T}$ are small compared with the major power components and $c$ an be set equal to zero.

Under steady state conditions the plug pumping rate must balance the plug filling rate

$$
\left(\frac{g-1}{g}\right) f_{C x}^{p} I_{b}^{p}=I_{t r a p}
$$

where $I_{\text {trap }}$ is the plug barrier filling current and $(g-l) / g$ is the fraction of the charge exchange events which occur with trapped particles. Itrap is taken as an input parameter in the solution of the particle and power balance equations and in these equations we replact ${ }_{-x}^{p} I_{b}^{P}$ with $(g / g-1) I_{\text {trap }}$ and $f_{i}^{P} I_{b}^{P}=f_{i}^{P} / f_{c x}^{p} \cdot f_{c x}^{p} I_{b}^{P}=0.4(g / g-1) I_{\text {trap }}$ 
With these simplifications and assumptions, for steady state conditions:

Cold-Ion Particle Balance

$$
\begin{gathered}
0.5 I_{\text {gas }}^{\text {rad }}+I_{d r a g}+\frac{2.8 g}{g-} I_{\text {trap }} f_{c i r c}-I_{\text {past }}^{\text {ion }}-I_{\text {rad }} \\
-\frac{2 g}{g-T} I_{\text {trap }}-\frac{n_{c}}{n_{e}} f_{c x}^{c} \int_{b}^{c}=0
\end{gathered}
$$

Cold-Ion Power Balance

$$
\begin{aligned}
P_{i h}+ & P_{\text {ICRH }}+I_{d r a g} \phi_{c}+\frac{2.8 g}{g-1} I_{\text {trap }} f_{c i r c} \bar{E}_{c i r c}-0.5 I_{\text {gas }}^{\text {rad }}\left(3 / 2 T_{i}\right) \\
& -\left(I_{\text {rad }}+\frac{2 g}{g-1} I_{\text {trap }}+\frac{n_{i c}}{n_{e c}} f_{c X}^{c} I_{b}^{c}\right)\left(3 / 2 T_{i}\right)-I_{\text {post }}^{\text {ion }}\left(\phi_{i}+T_{i}\right)-P_{i e}=0
\end{aligned}
$$

Hot-Ion Particle Balance

$$
\left(f_{i}^{c}+f_{c x}^{c}\right) I_{b}^{c}-\left(\frac{n_{i h}}{n_{e c}}\right) f_{c x}^{c} I_{b}^{c}-I_{d r a g}=0
$$

Hot-Ion Power Balance

$$
\left(f_{i}^{c}+f_{c x}^{c}\right) I_{b}^{c} \cdot E_{i n j}-\left(\frac{n_{i h}}{n_{e c}}\right) f_{c x}^{c} I_{b}^{c} \cdot \bar{E}-P_{i h}-P_{e h}-I_{d r a g} \phi_{j}=0
$$

Thermal-Electron Particle Balance

$$
I_{\text {past }}^{\text {electron }}=I_{11}^{\text {ion }}+I_{\text {rad }}+\frac{2.4 \mathrm{~g}}{(g-1)} I_{\text {trap }}\left(1-f_{\text {circ }}\right)
$$

Thermal-Electron Power Bal ance

$$
P_{H e}+P_{i e}-I_{\text {past }}^{\text {electron }}\left(\phi_{e}+T_{e d}\right)=0
$$


(We ignore the term $0.2(\mathrm{~g} / \mathrm{g}-1) \mathrm{I}_{\text {trap }} \phi_{\mathrm{e}}$ which is the input of cold electrons introduced by ionization of the pump beam on the central cell side of the pilgg and for TMX-U is of the order of $10 \mathrm{~kW}$.

We assunie the same central-cell geometry as the positive case and scale the ionization and charge exchange efficiencies with density to obtain

$$
\begin{aligned}
& f_{i}^{c}=6.5 \times 10^{-15} n_{e c} \\
& f_{c x}^{c}=1.3 \times 10^{-14} n_{e c}
\end{aligned}
$$

Solutions of these equations for input conditions

$$
\begin{aligned}
& n_{i c}=1.06 \times 10^{13} \\
& T_{i}=1 \mathrm{keV} \\
& \phi_{i} / T_{i}=2.5 \\
& I_{\text {trap }}=17 \text { amps } / \mathrm{p} 1 \mathrm{ug} \\
& A=1 \text { (hydrogen) } \\
& r_{c}=26 \mathrm{~cm} \\
& { }_{{ }_{c i r c}}=0.3 \\
& I_{c \text { irc }}=4.0 \mathrm{keV}
\end{aligned}
$$

and for the TMX-U magnetic field parameter are given in Table 10-1 for three cases:

- Case A - Resonant transport same as positive case.

- Case 8 - No resonant transport.

- Case C - No resonant transport. ICRH heating of the central-cell plasma instead of neutral beams. 
$-156=$ 


\section{REFERENCES - SECTION 1}

1. F. H. Coensgen, T. C. Simonen, A. K. Chargin, and B. G. Logan, TMX Major Project Proposal, Lawrence Livermore National Laboratory, Livermore, CA, LLL-Prop-172 (198C)

2. D. E. Baldwin, Physics Bas is for MFTF-8, Lawrence Livermore National Laboratory, Livermore, CA, UCID-18496 (1980).

3. R. Bardet, P. Briand, L. Dupas, C. Gormezano, and G. Meilin, Nucl. Fusion 15, 865 (1975).

4. W. A. Perkins and W. L. Barr, Phys. Fluids 11, 388 (1968).

5. TMX Group, T. C. Simonen, Ed., Surmary of Results from the Tandem Mirrar Experiment (TMX), Lawrence Livermore National Laboratory, Livermore, CA, UCRL-53120 (1981).

6. B. G. Logan, Lawrence Livermore National Laboratory, Livermore, CA, private communication, 1981.

7. Mirror League Workshop, Lawrence Livermore National Laboratory, Livermore, CA (1979).

REFERENCES - SECTION 2

1. B. W. Stallard, Y. Matsıda, and W. M. Nevins, Fokker-Planck Calculations of Electron Cylcotron Resonant Heating (ECRH) in Mirror Geometry, Lawrence Livermore Nationa 1 Laboratory, Livermore, CA, UCRL-86826 (1980).

2. B. Boghosian, R. B. Campbell, and 3. M. Gilmore, A Potential Solver for Sloshing-Ion Therma1 Barriers, Lawrence Livermore National Laboratory, Livermore, CA , UCID-19215 (1981).

3. B. MCNamara et a1., Theory of Mirror Machines at High Beta, IAEA-CN-35/C3 (1978).

4. L. L. LoDestro, Sleady-State Ion Distributions in a Potential and Magnet ic We 11, Lawrence Livermore National Laboratory, Livermore, CA, Mirror Theory Monthiy (May 25, 1980).

5. L. L. LoDestro, D. E. Baldwin, T. A. Cutler, B. G. Logan, and T. D. Rognlien, Bu11, Amer. Phys. 5oc, 24, 1060 (1979).

6. R. H. Cohen, Axial Potential Profiles in Thermal-Barrier Cells, Lawrence Livermore Nationa 1 Laboratory, Livermore, CA, UCRL-84535 (1980); submitted to Nucl. Fusion. 
REFERENCES - SECTION 3

1. L. L. Lodestro, Steady-State Ion Distributions in a Potential and Magnetic We1l, Lawrence Livermore National Laboratory, Livermore, C.A, Mirror Theory Monthly (May 25, 1980).

2. G. 0. Porter, Analytical Model for the Trapping Rate of Passing Particles in a Thermal Barrier, Lawrence Livermore National Laboratory, Livermore, CA, memo MFE/CP/81-3505m/0404m (1981); a7so, Archer Futch is in the process of publishing a fit to the $F . P$. code results.

3. T. C. Simonen et al., Re-evaluation of the TMX-Upgrade Pump-Beam Current Requirements, Lawrence Livermore National Laboratory, Livermore, CA, memo, Conf. 80-239 (October 28, 1980).

REFERENCES - SECTION 5

i. B. W. Stallard, Y. Mats:;a, and W. M. Nevins, Fokker-Planck Calculations of Electron Cylcotron Resonant Heating (ECRH) in Mirror Geometry, Lawrence Livermore National Laboratory, Livermore, CA, UCRL-86826 (1980).

2. F. H. Coensgen, T. C. Simonen, A. K. Chargin, and B. G. Logan, TMX Major Project Proposal, Lawrence Livermore National Laboratory, Livermore, CA, LLL-Prop-172 (1980)

3. K. Bardet, P. Briand, L. Dupos, C. Gormezano, and G. Melin, Hot-Electron-P lasma Accumulation in the CIRCE Mirror Experiment, Nucl. Fusion 15, 865 (1976).

4. H. B. Ard et al,, "Use of a Hot-Electron Target Plasma for Accumulation of Energetic Ions in Stabilized Magnetic Mirror Traps," in Proc. Conf. on Plasma Physics and Controlled Nuclear Fusion Research, 4th, Madison, Wisconsin, 1971 (IAEA, 1971), p. 619-628.

5. R. Dandl, Lawrence Livermore National Lavoratory, Livermore, CA, internal memo (Sept. 1979).

6. D. B. Batchelor, Lawrence Livermore National Lavoratory, Livermore, CA, private communication (1981).

3. TRW, Symmetric Tandem Mirror Program Annual Progress Report, TRit Repart, $S / N-35638(1980)$. 
8. 0. A. Anderson, B. H. Birdsall, C. W. Hartman, E. B. Hooper, Jr., and R.

$H$. Munger, Microwave Heating of Hot Electron Plasma in the

Superconduct ing Levitron, Lawrence Livermore National Latoratory,

Livermcre, CA, UCRL-73984 (1972); also Annual Report, Ibid,

UCRL-50002-73 (1973).

REFERENCES - SECTION 6

J. G. E. Guest and D. J. Sigmar, Nuc1. Fusion 11, 151 (1971).

2. 3. Jacquinut and C. Leloup, Phys. Fluids 14, 2440 (1971).

3. N. T. Gladd et al., "Microstability of the EBT Boundary," N. A. Uckan, Ed., in Proc. Horkshop on EBT Stability Theory, Oak Ridge, TN, 1981; Section 4.

$\therefore$ N. T. Gladd et al., "The Whistler Instability at Relativistic Energies," N. A. Uckan, Ed., in Proc. EBT Ring Physics Workshop, 2nd, San Diego, CA, 1981.

5. M. Ashour-Abdalla and C. F. Kennel, 3. Geophys. Res. 83, 1531 (1978).

6. Yu-Juian Chen, W. M. Nevins, and G. R. Smith, in Proc. Workshop on Hot Electron Ring Physics, 2nd, San Diego, CA, 1981.

7. T. S. T. Young, J. D. Callen, and J. E. McCune, J. Geophys. Res. 78, 7082 (1973).

8. J. E. Scharer, Phys. Fluids 10, 652 (1967).

9. Cuperman and Landau, UGR 79, 128 (1974).

10. C. L. Hedrick, Oak Ridge National Laboratory, Oak Ridge, TN, ORNL-TM-3143 (1970); unpub) ished.

11. E. A. Robertson and A. Bers, Massachusetts Institute of Technology, Boston, MA, MIT Quarteriy Progress Report No. 79, P. 111 (1965).

12. R. A. Dandl et al., Madison, HI, IAEA-CN-281G-4, p. 607 (1971).

13. M. Hosokawa and H. Ikegami, ir Proc. EBT Ring Physics Workshop, Oak Ridge, TN (1979), p. 59; H. Ikegami et a1., IAEA-CN-24/J-5 (1968) p. 423; and H. Ikegami et a]., Phys. Fiuids 11, 1061 (1968).

14. W. A. Perkins and W. L. Barr, Phys. Fluids 11, 388 (1968); IAEA-CN-21/93 (1966) p. 115.

15. C. E. Wagner, I. M. Lidsky, and R. A. B lanken, Phy5. Fluids 14, 2447 (1971).

16. V. V. Alikaev, V. M. Glagolev, and S. A. Moroyov, Plasma Phys. 10, 753 (1968). 
17. R. ". Blanken and N. H. Lazar, Phys. Fluids 13, 2752 (1970).

18. S. J. Gitower and 3. L. St shet, Phys Fluids, 13, 413 (1970).

19. M. J. Schwartz and A. J. Lichtenberg, Phys. Fluids 15, 470 (1972).

20. J. ' acquinot et a I., IAEA-CN-24/H-1 I, Vol. II, p. 347 (1969).

21. R. Bardet, P. Briand, L. Dupas, C. Gormezano, and G. Melin, Nuc1. Fusion 15, 865 (1975).

22. T. A. Casper, W. H. Nevins, and G. R. Smith, "Microstability

Considerations for a Negative Tandem Mirror, "Post-Deadiine Session, Amer. Phys. Soc., New York, 1981.

23. B. W. Stallard, Y. Matsuda, and W. M. Nevins, "Fokker-Planck

Calculations of Electron Cyclotron Resonant Heating (ECRH) in Mirror

Geometry," N. A. Uckan, Ed., in Froc. EBT Ring Physics Workshop, 2nd, San Diego, CA, 1981.

24. J. D. Callen and G. E. Guest, Mucl. Fusion 13, 87 (1973).

RI ERENC:S - SECTION 7

1. J. W. Van Dam and Y. C. Lee, in Proc. EBT Ring Physics Workshop, oak Ridge, TN (1979), p. 471 .

2. u. H. Foote et a1., TMX-Upgrade Magnet System - Design Characteristics and Physics Considerations, Lawrence Livermore National Laboratory, Livermore, CA, UCRL-86677 (1977).

REFFRENCES - SECTION 8

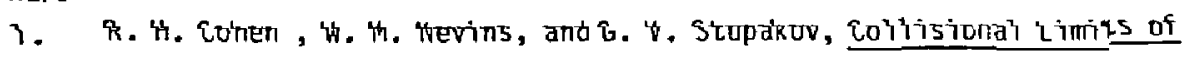
Resonant and Neoclassical Transjort in Tandem Mirrors, Lawrence Livermore National Laboratory, -ivermore, CA, UCRL-86752 (1981).

2. R. P. Drake et a 1., A Detailed Study of Radial Transport in the MX Central Ce11, Lawrence Livermore National Laboratory, Livermore, CA, UCRL-85872 (1981).

REFERENCES - SECTION $g$

i. B. W. Stallard, Y. Matsuda, and W. M. Nevins, Fokker-Planck Calcullations of Electron Cyclotron Resonant Hcating (ECRH) in Mirror Geometry, Lawrence Livermore National Laboratory, Livermore, CA, UCRL-86826 (1981).

2. T. C. Simonen, TMX-Upgrade 5tar:-Up Guns, Lawrence Livermore Nationa1 Laboratory, Livermore, CA, Memorandum MFE/CP/80-2573t (1981). 
3. J. E. Osher, Plasma Target Output from a Magnetically-Augmented, Gas-injected, Washer-Stack Plasma Gan, Lawrence Livermore National Laboratory, Livermore, CA, UCRL-84480, Rev. 1 (1981); also private communication.

4. G. G. Kelley, Plasma Phys. 9, 503 (1967).

5. D. E. Baldwin, Physics Bas is for MFTF-B, Lawrence Livermore Nationa1 Laboratory, Livermore, CA, UCID-18496, Part 2 (1980) P. IV-79.

6. D. E. Baldwin, Same Effects of Hot Electron Stability in Tandem Mirror Geometry, Lawrence Livermore National Laboratory, Livermore, CA, Mirror Theory Monthly (February 15, 1982).

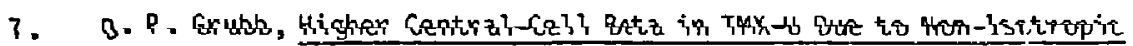
pressure Profiles, Lawrence Livemore National Laboratory, Livermore, CA, Memc andum PJR/3676r/0425r (1982).

8. L. D. Pearlstein, T. B. Kaiser, and W. A. Newcomb, Phys. Fluids 24, 1325 (1981).

9. F. H. Coensgen, TMX-Upgrade Major Project Proposal, Lawrence Liverinore fiational Laboratory, Livermore, CA, LLL-Prop-172 (1980).

REFERENIES - AFPENDIX A

1. D. P. Grubb and B. W. Stallard, Analytic Formulas for Electron Power and Particle Balance in TMX-U, Lawrence Livermore National Laboratory, Livermore, CA, Memorandum MFE/CP/a2-3712/0424m (1982).

2. $Y$, Matsuda, Lawrence Livermore National Laboratory, Livermore, CA, private communication (1982).

$\mathrm{BS} / \mathrm{mm}$ 